\title{
A University Consortium on Homogeneous Charge Compression Ignition Engine Research
}

\author{
DE-FC04-02AL67611 \\ Final Report \\ June 1, 2007 \\ Participating Universities \\ University of Michigan \\ Massachusetts Institute of Technology \\ Stanford University \\ University of California, Berkeley \\ Participating Investigators (in alphabetical order) \\ Dennis N. Assanis* (UM) \\ Arvind Atreya (UM) \\ Craig T. Bowman (SU) \\ Jyh-Yuan Chen (UCB) \\ Wai K. Cheng (MIT) \\ David F. Davidson (SU) \\ Robert W. Dibble (UCB) \\ Chris F. Edwards (SU) \\ Zoran S. Filipi (UM) \\ David M. Golden (SU) \\ William H. Green, Jr. (MIT) \\ Ronald K. Hanson (SU) \\ J. Karl Hedrick (UCB) \\ John B. Heywood (MIT) \\ Hong G. Im (UM) \\ George A. Lavoie (UM) \\ Volker Sick (UM) \\ Margaret S. Wooldridge (UM)
}

* Principal Investigator to whom all correspondence should be addressed:

Prof. Dennis N. Assanis

Department of Mechanical Engineering

University of Michigan/2043 WE Lay Auto Lab

Ann Arbor MI 48109-2121

(734) 763-7880 (Office), (734)764-4256 (Fax), assanis@ @umich.edu 


\section{DISCLAIMER}

This report was prepared as an account of work sponsored by an agency of the United States Government. Neither the United States Government nor any agency thereof, nor any of their employees, makes any warranty, express or implied, or assumes any legal liability or responsibility for the accuracy, completeness, or usefulness of any information, apparatus, product, or process disclosed, or represents that its use would not infringe privately owned rights. Reference herein to any specific commercial product, process, or service by trade name, trademark, manufacturer, or otherwise does not necessarily constitute or imply its endorsement, recommendation, or favoring by the United States Government or any agency thereof. The views and opinions of authors expressed herein do not necessarily state or reflect those of the United States Government or any agency thereof. 


\section{Summary}

Over the course of this four year project, the consortium team members from UM, MIT, Stanford, and Berkeley along with contributors from Sandia National Labs and LLNL, have produced a wide range of results on gasoline HCCI control and implementation. The work spanned a wide range of activities including engine experiments, fundamental chemical kinetics experiments, and an array of analytical modeling techniques and simulations. Throughout the project a collaborative approach has produced a many significant new insights into HCCI engines and their behavior while at the same time we achieved our key consortium goal: to develop workable strategies for gasoline HCCI control and implementation. The major accomplishments in each task are summarized below, followed by detailed discussion in the following pages.

\section{Task 1: Development of engine control systems}

On both single and multi cylinder test engines MIT, Stanford, and Berkeley researchers have utilized variable valve actuation (MIT, SU) and exhaust throttles and intake charge heaters (UCB) to control the amount of residual gas in the cylinder and the combustion timing. The experiments characterized operating ranges and identified robust and non-robust transitions between SI and HCCI mode. Successful strategies for load and combustion phasing control have also been developed using LQR and other controllers and demonstrated in engine testing. These tests primarily have used pressure transducers as combustion timing sensors but also have successfully demonstrated an ion probe sensor.

Detailed heat transfer measurements at UM have shown that heat transfer in HCCI engines is different from either gasoline or diesel engines. The effects of flame travel and radiation are greatly reduced in the near-homogeneous combustion that characterizes HCCI. To take this into account a new heat transfer correlation was developed and has been employed in the engine modeling work throughout the consortium

Also at UM it was discovered that wall temperature has a large effect on HCCI combustion timing, particularly in engines employing large amounts of Residual Gas Fraction (RGF) for HCCI ignition, which may be on the order of 50\%. Because engine wall temperature changes much more slowly than typical engine speed and load transitions, the optimal RGF levels for a given operating point will depend on the wall temperature history rather than the value obtained in the corresponding steady state dynamometer test. System model simulations confirmed that the stable HCCI operating range in the speed load map is highly dependent on the instantaneous wall temperature. The study concluded that in order to fully benefit from HCCI operation, additional sensors and control algorithms will be needed relating to the thermal state of the engine.

\section{Task 2: Full cycle and system modeling tools}

UM modelers, together with collaborators at LLNL have developed a computationally efficient technique for carrying out fully coupled KIVA-Kinetic calculations throughout the engine cycle. This involves a new method of mapping and remapping physical space into a smaller chemical space in which the detailed kinetic calculations are performed. Parametric studies on burn rate and combustion efficiency with the new model are proving invaluable in 
evaluating effects of mixing and temperature gradients on combustion. The new model is also being used to generate "data sets" with which to develop "smart" correlations of burn rate properties for use as submodels for system simulation tools and to supplement experimental data.

The accuracy of simple auto-ignition integral techniques was compared to detailed kinetic methods for determining ignition time. The results showed similar behavior over a range of RPM and inlet temperatures. This supports the widespread use of knock integrals as a suitably fast algorithm for full engine and vehicle simulations, and provided useful insight into the scaling of combustion rates and ignition timing.

A fast GT-Power engine system model was developed at UM incorporating the new heat transfer model for HCCI, a knock integral model for ignition timing and an empirical data fit for burn rate and combustion efficiency. This model was used extensively in the thermal transient studies in Task 1.

\section{Task 3: Chemical kinetics for HCCI combustion}

Investigators at all four universities completed a fundamental characterization of isooctane combustion and ignition delays in the pressure, temperature and composition regimes of interest. The experiments in the UM Rapid compression Facility (RCF) and in Stanford shock tubes along with modeling work at MIT and Berkeley have provided a consensus on practically efficient models for isooctane ignition. Further, useful correlations of the data have been developed which have been used in auto-ignition integral models handed off to Task 2 for system simulations.

Shock tube work at Stanford on gasoline has shown that in the HCCI pressure and temperature regime gasoline fuel appears to behave substantially like isooctane. This supports the successful practice of using isooctane kinetics to represent gasoline combustion engines. We believe that, at least for system level models, this will be sufficiently accurate.

\section{Task 4: Detailed studies of mixing and reaction}

Results from the one-dimensional counterflow UM model suggest that the effects of scalar dissipation rate is rather insignificant for HCCI conditions with gas-phase mixing or early injection conditions, in which case the flamelet equation degenerates to the multi-zone model. However, the scalar dissipation rate can play a crucial role in determining the ignition of the HCCI engines when combustion is triggered by a secondary injection for controlling start of combustion or under DI conditions, i.e. conditions in which significantly larger local gradients of temperature and concentration occur.

A sequential multi-zone CFD model was used to explore the mixing of Residual Gas Fraction in the cylinder. The mixing effects were investigated by characterizing the mixture as "clouds" which evolve in composition-temperature space during the inlet and compression process. The results showed that under marginal combustion conditions the degree of unmixedness was influenced the combustion event through its effect on the cold temperature regions where there is relatively little residual gas and near the wall. Under these conditions exhaust rebreathing valve timing yields more homogeneous mixtures than recompression valve 
timing, particularly at high levels of RGF. For more robust combustion conditions the variations in distributions were less significant.

In other work, Planar Laser Induced Fluorescence (PLIF) has been used to observe mixture homogeneity in an HCCI engine with exhaust rebreathing. The results show that the spatial inhomogeneity increases toward TDC. Finally, the Tunable Diode Laser technique developed at Stanford for simultaneously measuring in-cylinder temperatures and water concentration was demonstrated in the UM optical engine. This diagnostic will provide a tool for calibrating cycle models for EGR and temperature. 


\section{Task 1: Development of Engine Control System}

\section{Managing HCCI Mode Transition (MIT)}

The research focused specifically on gasoline HCCI engines employing Variable-ValveTiming (VVT) systems to control engine operation. In these engines, the valve timings are set up so that a large amount of hot residual is trapped in the cylinder. The hot residual is used as means of controlling the charge temperature necessary for HCCI combustion. These engines are often referred to as Controlled-Auto-Ignition (CAI) engines.

The range of operation of gasoline HCCI engine is limited by knocking or misfiring at high load, and combustion failure at low load. To cover the entire vehicle operating range, it is therefore necessary to equip a vehicle with a dual mode engine which can switch between SparkIgnition (SI) and HCCI operations. Furthermore, for CAI engines which employ trapped hot residual as means of initiating combustion, it is necessary to operate the engine in SI mode first. Thus the HCCI mode is used at part and medium loads and the SI mode is used at cold start and high loads. As such, understanding the engine behavior in mode transitions and the management of these transitions are critical to the successful implementation of these engines.

\section{Consideration of mode transition}

To assess the load/speed conditions encountered in vehicle operation, we considered the US Federal Testing Procedure (FTP) Urban Fuel Economy Drive Cycle. The second-by-second operating points for a mid-size vehicle (Ford Taurus) in the cycle are shown in Fig. 1.1. Also shown in the figure is the boundary of HCCI operation for a CAI engine reported in the literature. The corresponding points on a speed/time curve are shown in Fig. 1.2. It is noted that only $40 \%$ of the operating points are within the HCCI operating regime (the value is $75 \%$ for the EPA Highway Fuel Economy Cycle), and that mode transitions are frequent. Furthermore, when these transitions occur is not predictable if the driving cycle is unknown.

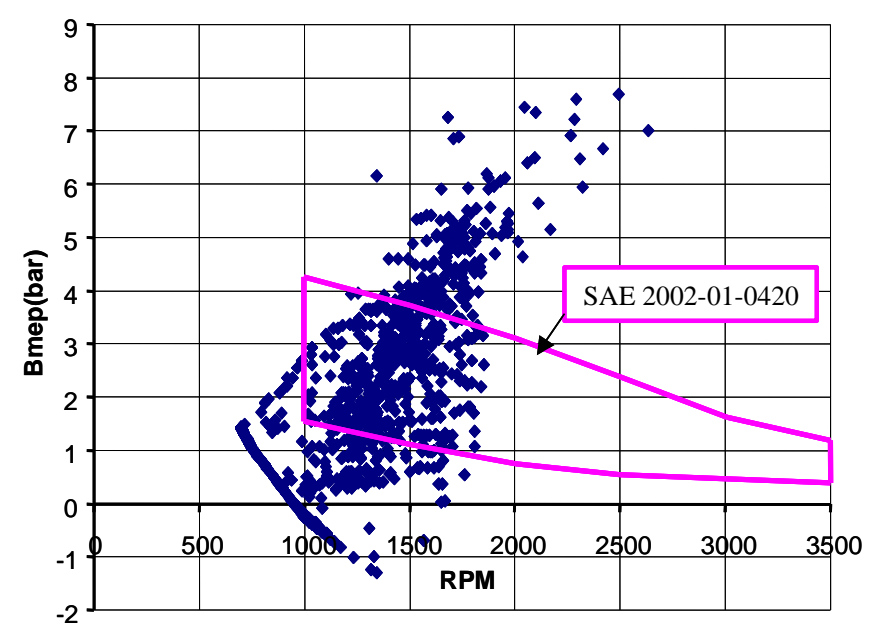

Fig. 1.1 Second-by-second operating points on the engine map for the FTP drive cycle. The HCCI operating boundary according to Ref. 3 is also shown.

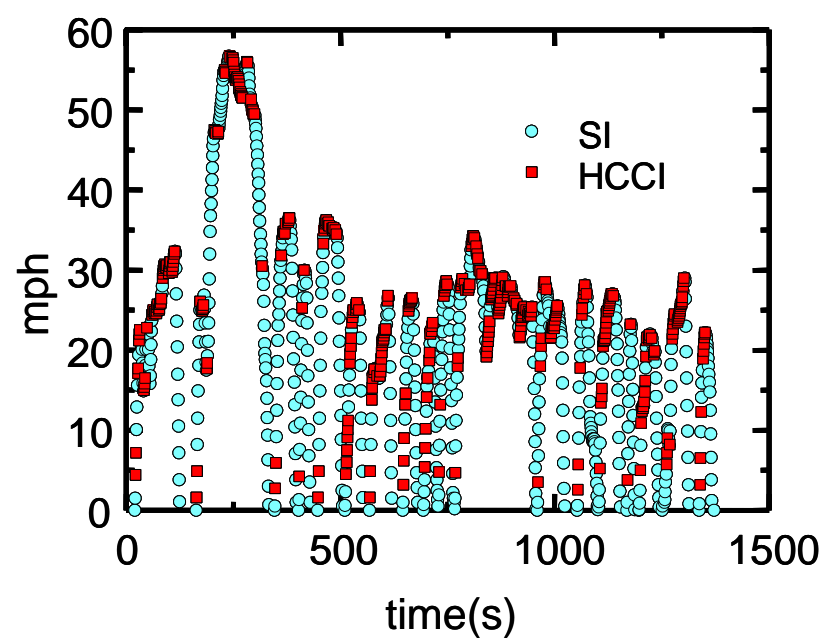

Fig. 1.2 Second-by-second speed history of vehicle in the FTP cycle with dual SI/HCCI mode of operation. 


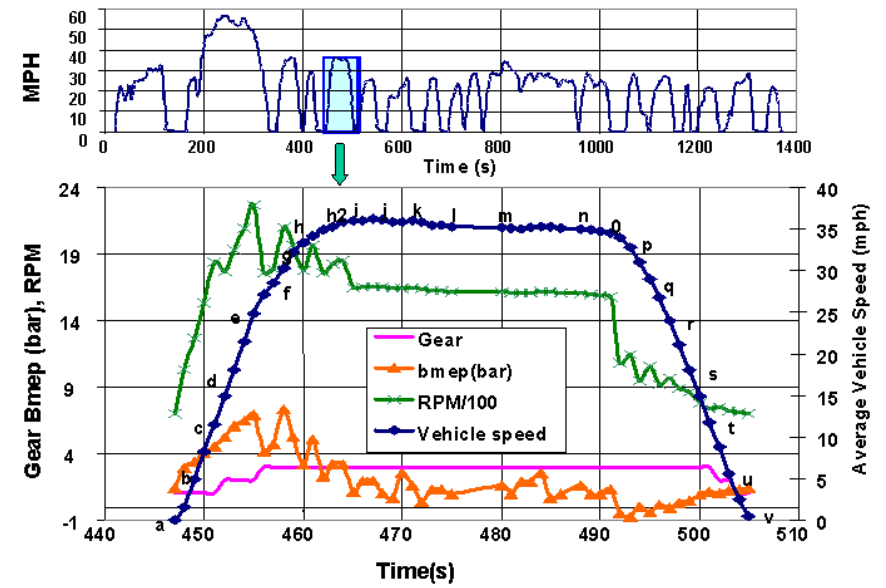

Fig. 1.3 Second-by-second engine operating conditions in the $5^{\text {th }}$ cycle of the FTP.

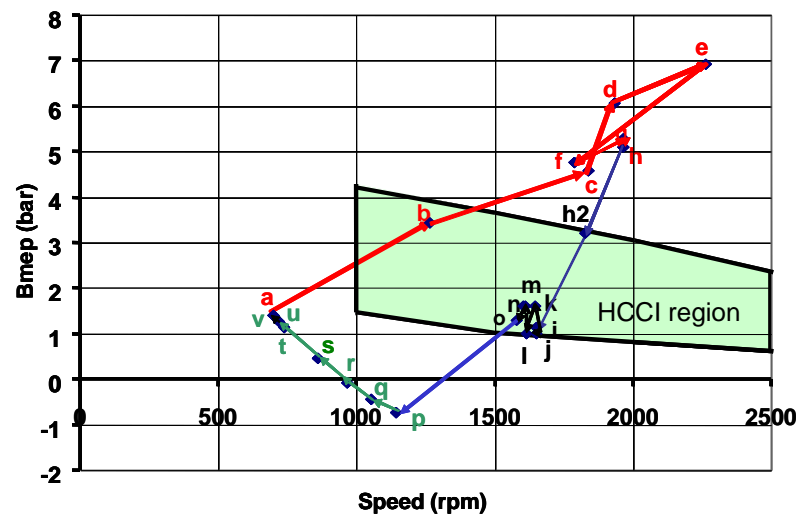

Fig. 1.4 Engine load-speed trajectory in the $5^{\text {th }}$ cycle of FTP.

To illustrate the dynamic nature of mode transition, the detailed second-by-second engine operating condition for the $5^{\text {th }}$ cycle of FTP is shown in Fig. 1.3. The corresponding load-speed trajectory on the engine map is shown in Fig. 1.4. It is noted that the mode switching operation is very dynamic. For example, starting from the idle condition (a) which is in the SI regime, the engine accelerates; it rapidly traverses the HCCI light-load region along trajectory (a)-(b)-(c) (the points are at 2 seconds apart) and returns to the SI regime again for the higher load operation. For this case, the residence time in the HCCI regime is only 2 seconds, which, at the average speed of approximately $1200 \mathrm{rpm}$, corresponds to only 20 cycles of firing in each cylinder. It will be shown (in the Mode Transition Phenomena section) that the engine takes tens of cycles to stabilize after a mode transition. Thus a load following control strategy is necessary for operating the engine within the HCCI regime.

When the vehicle reaches cruise speed, the high torque required for acceleration is no longer required. Then the engine goes back into light-load operation and stays in the HCCI regime (points (h2) to (o) in Figures 1.3 and 1.4). It is noted that the cruise operating points (m)(o) are quite close to the operating boundary of the HCCI regime. Therefore, for a meaningful HCCI operation in a vehicle, the lower load boundary must be of sufficiently low value to encompass cruise operation.

In general, mode transition from HCCI to SI regime does not present difficulty because the SI engine combustion is relatively independent of the conditions of the preceding cycle. In contrast, since a CAI engine relies on the trapped hot residual as means to enable HCCI combustion, there is significant dependence of the current cycle behavior on the preceding one. In particular, for SI to HCCI mode transition, not only must the trapped residual from the last SI cycle be able to render compression ignition in the first HCCI cycle, but also that from every subsequent cycle has to do the same for the next cycle. The requirement is especially difficult to meet if the mode transition is to occur immediately after the load-speed trajectory crosses the regime boundary from an idle/light-load SI operation. Thus for robust transition, SI to HCCI 
mode switching should occur at an operating point at a higher load which is beyond the steady state operating boundary of the HCCI regime.

In summary, the behavior of a dual mode SIHCCI engine in a vehicle is very dynamic. Typical urban drive cycle requires frequent mode transitions at not predictable times. The residence time of the engine operating point within the HCCI regime could Table 1.1 Engine and fuel specifications be from a few seconds to tens of seconds. Appropriate

\begin{tabular}{|l|l|}
\hline Bore & $80.26 \mathrm{~mm}$ \\
\hline Stroke & $88.9 \mathrm{~mm}$ \\
\hline Connecting rod length & $158 \mathrm{~mm}$ \\
\hline Displacement & $449.8 \mathrm{cC}$ \\
\hline Compression ratio & 12.3 \\
\hline Intake air temperature & $120^{\circ} \mathrm{C}$ \\
\hline Fuel & Chevron Philips UTG91 \\
\hline Octane \# & RON 91; MON 83 \\
\hline PFI injection pressure & 2.7 bar differential \\
\hline
\end{tabular}
engine system design (e.g. employing a hybrid configuration) may be devised to minimize the number of transitions; nevertheless mode transitions are inevitable. Thus it is important to assess the mode transition behavior of this type of engine.
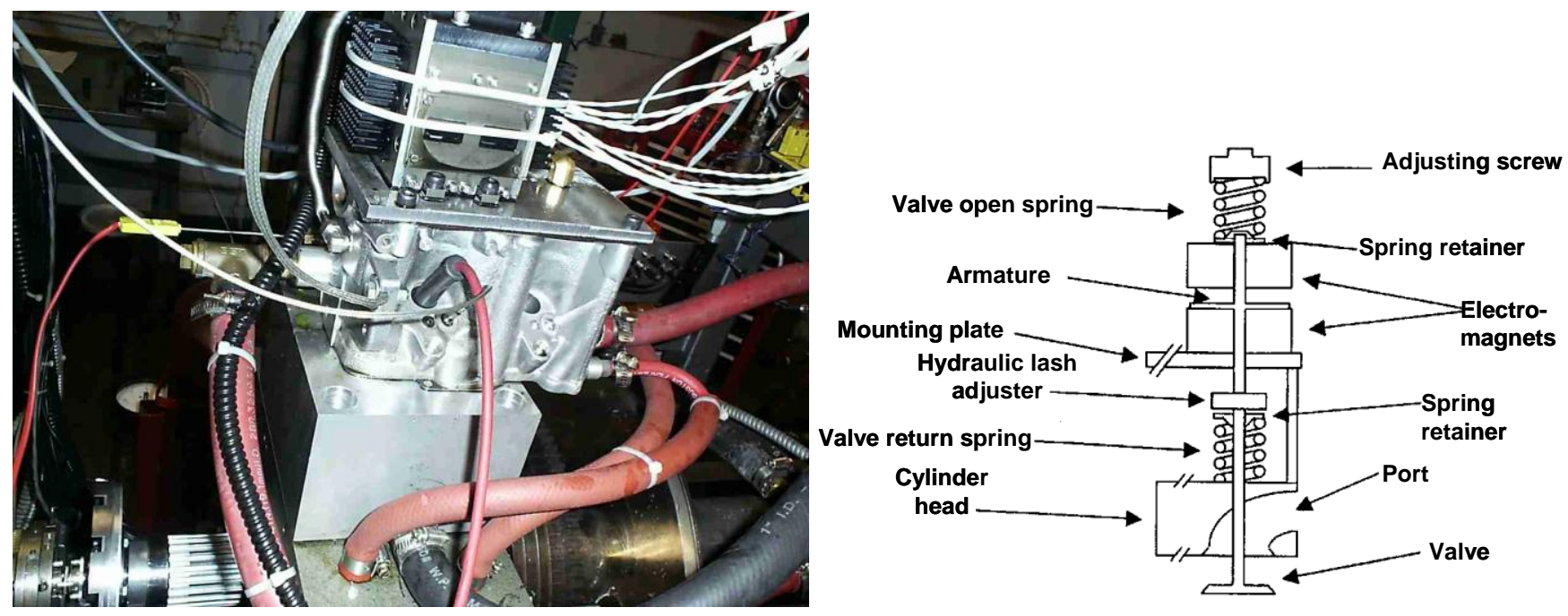

Fig. 1.5 Single cylinder dual mode SI-HCCI engine with electromagnetic VVT system.

\section{Approach}

A single cylinder engine employing a modified VW-TDI head mounted on a Ricardo Hydra block was used in this study. With an electromagnetic VVT device, the valve timings (IVO, IVC, EVO, EVC) were all independently commanded via a National Instrument PXI embedded computer which was controlled by a desktop computer. The electromagnetic VVT was chosen as the means of controlling the engine because it could provide control action on a cycle by cycle basis.

The engine is shown in Fig. 1.5. The engine and fuel specifications are shown in Table 1.1. The intake air was heated so that there was a sufficient large range of operation for testing of the control capabilities. Fuel was injected in the port. 


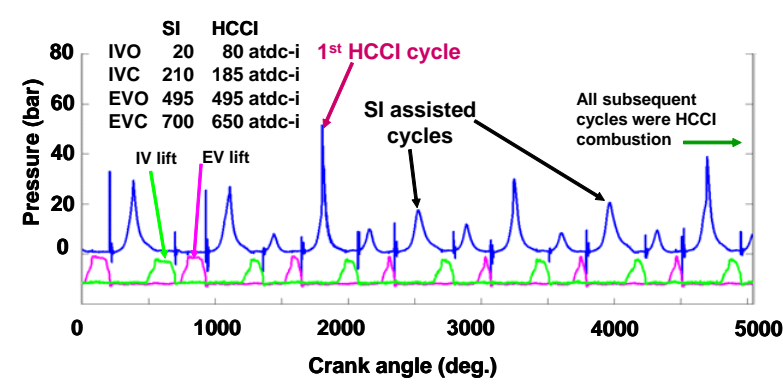

Fig. 1.6 A non-robust SI-HCCI transition. The valve timing and fuel amount were step-changed from the SI values to the HCCI values. Engine at $1500 \mathrm{rpm}$.

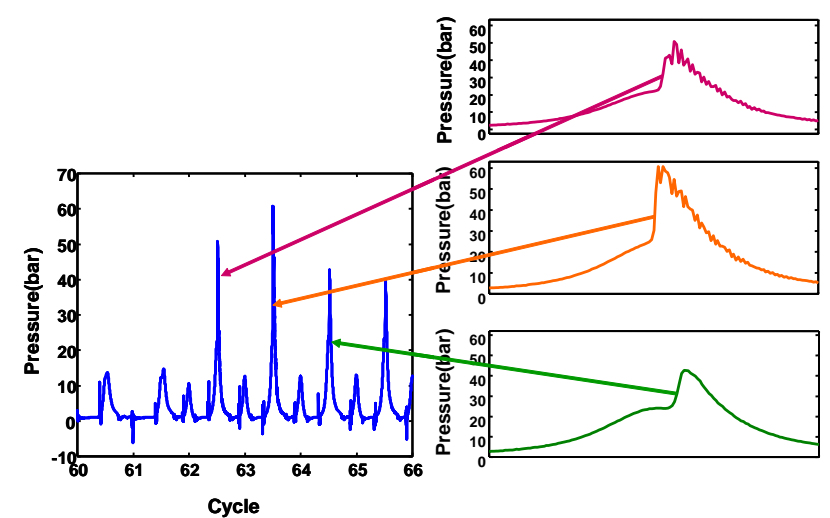

Fig. 1.7 A knocking SI-HCCI transition. The first two cycles knocked before settling down into stable HCCI operation. Engine at $1500 \mathrm{rpm}$.

\section{Mode transition behavior}

The SI to HCCI transition process may be classified into the following categories. Note that in the context of this discussion, the spark ignition was on all the time so that if a cycle failed to go into HCCI combustion, it could still operate in the SI mode to provide hot residual gas for HCCI combustion in the subsequent cycle. Our spark timings were set at MBT in the SI mode, and at TDC of the compression stroke in the HCCI mode. The latter timing was employed so that if the HCCI operation was successful, it would not be interfered by the spark discharge.

Transition into HCCI mode was not successful by directly jumping into the steady state HCCI settling, although the load/speed point could operate in HCCI mode in steady state by transitioning into other points in the HCCI region first and then by moving to that point with a load/speed trajectory within the HCCI region.

\section{Non-Robust transition}

This may be subdivided into the following cases:

a) There may be one or more missed cycles (i.e. combustion in the SI mode) before settling into HCCI operation. - An example of non-robust transition is shown Fig. 1.6. Here, the transition was implemented by step-changing the valve timing (listed on the figure) and fuel amount from the SI values to the HCCI values. In the last SI cycle, the EVC timing was changed to the retarded value. A re-compression peak could be observed in the pressure trace. In the first HCCI cycle, the IVC was advanced. The HCCI timing values and fuel amount had been previously established to provide stable HCCI combustion at the same speed and NIMEP by steady state engine mapping. The first cycle after the step change operated successfully in the HCCI mode. The second cycle, however, failed; the charge was ignited by the spark at TDC. (The pressure trace of the "spark-assisted" cycles had distinctively different signature than that of the HCCI cycles.) The third cycle operated in the HCCI mode successfully. Then the fourth cycle failed again. From the fifth cycle on, however, the engine settled into HCCI combustion in every cycle. 
b) One or more knocking cycles in the transition. The situation is illustrated in Fig. 1.7. Here the transition scheme was a stepchange in valve timing from the SI mode to the HCCI mode similar to that associated with Fig. 1.6. The first two HCCI cycles were knocking; then the engine settled into stable HCCI operation.

\section{Robust transition}

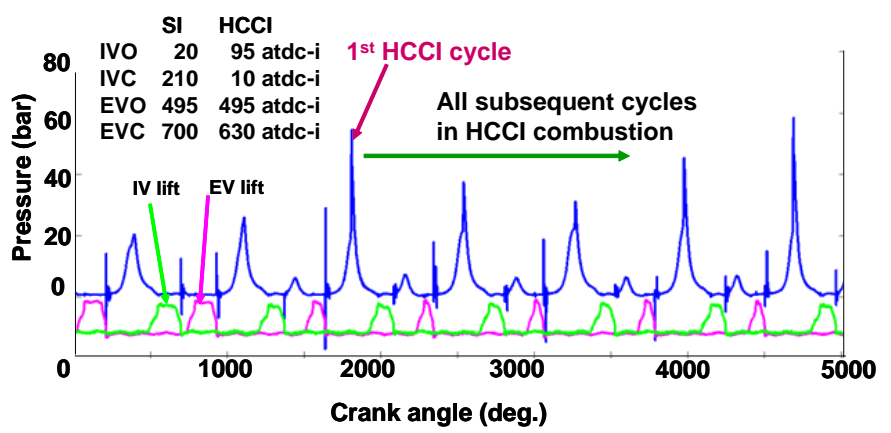

Fig. 1.8 A robust SI-HCCI transition. The valve timing and fuel amount were step-changed from the SI values to the HCCI values. All cycles went into HCCI combustion after the transition. Engine at $1500 \mathrm{rpm}$. Valve timing with respect to After-Top-Dead-Center of intake stroke.

The first and subsequent HCCI cycles all behave properly (i.e. complete combustion without excessive knocking.)

A robust SI-HCCI transition is shown in Fig. 1.8. Here every cycle after the step-change in operating condition was operating in HCCI mode.

The behaviors show that in order to have successful and robust mode transitions, it is necessary to manage the transition strategies carefully.

\section{Mode transition management}

From the above study of the mode transition phenomena, the following tranisiton management strategy for a SI-HCCI dual mode engine using VVT control was formulated. Here transition means a mode switch while maintaining the same value of engine speed and NIMEP output.

For the SI to HCCI transition:

(i) The switch point should be well inside the HCCI region at a location with robust mode transition.

(ii) The valve timing settings for all the speed/ NIMEP points within the HCCI region should be pre-mapped. Our experience was that intermediate values in between could be obtained by a linear interpolation of the valve timing values at the mapped points.

(iii) The transition could be managed by the valve timing setting. Prior to the transition, operate the part-load SI engine unthrottled and control the load by the IVC timing. The EVC timing would be set at the normal SI engine setting.

(iv) The HCCI engine is also operated unthrottled. To minimize pumping loss of the residual re-compression, the EVC and IVO timing are to be symmetric about the TDC of the intake stroke. 
(v) In the last SI cycle, switch over to early EVC to trap the hot residual for enabling HCCI. The setting should be the targeted EVC of the HCCI operating point.

(vi) In the first HCCI cycle, switch over to early IVC to increase the compression ratio and to control the amount of intake air. The

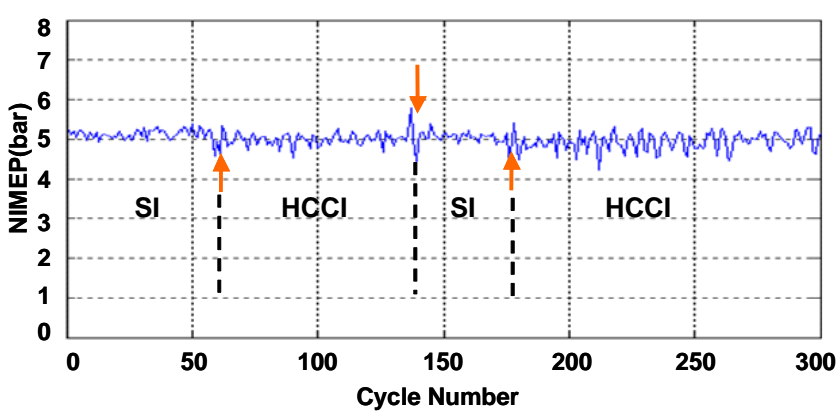

Fig. 1.9 Demonstration of repeated mode transitions of dual mode SI-HCCI engine at $1500 \mathrm{rpm}$ and 5 bar NIMEP. setting should be an intermediate value (approximately half way) between the last-cycle SI setting and the targeted HCCI setting.

(vii) To avoid a rich spike due to the change of the IVC timing, the fuel injection has to be reduced by a significant amount for the first HCCI cycle.

(viii) The EVC timing of the first and subsequent HCCI cycles are at the targeted value.

(ix) The IVC timing of the second and subsequent HCCI cycles are at the targeted value.

(x) The fuel per cycle for the second and subsequent cycles are at the targeted value.

For the HCCI to SI transition:

(i) For the last HCCI cycle, retard the EVC to the targeted SI mode setting.

(ii) For the first and subsequent SI cycle, set the IVC, EVC and spark timing to the targeted values.

(iii) For the first SI cycle, the injected fuel amount should be significantly higher than the targeted amount to avoid the lean spike due to the valve timing change. The fuel amount is to return to the targeted amount for the second and subsequent cycles.

Examples of successful SI to HCCI and HCCI to SI transitions are shown in Fig. 1.9. 


\section{Load following control within the HCCI regime}

To maximize fuel economy, the engine was run unthrottled. The IVC valve timing was used to control the trapped charge mass. To enable CAI, negative valve overlap was employed. To minimize the pumping loss in the residual gas re-compression process, the IVO timing was set to be symmetric to the EVC timing with respect to the TDC of the intake stroke. Thus the IVO timing was not an independent variable.

The EVO timing was deemed to play a relatively minor role in the engine operation; its effect was mainly through the exhaust system acoustic waves. Therefore the EVO timing was fixed at $135^{\circ}$ ATDC of the expansion stroke.

The control of the engine operation was therefore done via the following three quantities: injected fuel mass per cycle $\left(\mathrm{m}_{\mathrm{f}}\right)$, IVO timing, and EVC timing. These values were transformed into the control vector $\underline{u}$ as:

$$
\underline{u}=\left[\begin{array}{l}
\ln \left(m_{f} / m_{f, r e f}\right) \\
\ln \left(V_{I V C} / V_{\text {ref }}\right) \\
\ln \left(V_{E V C} / V_{\text {ref }}\right)
\end{array}\right]
$$

The use of the logarithmic transformation was based on the empirical power law pressure/volume dependence of the compression process. The logic runs as follows. The sensible enthalpy of the trapped charge during compression (but before any significant heat release) is one of the most important factors that determine the CAI combustion behavior. This value is roughly proportional to the product $\mathrm{pV}$ (it would be exactly proportional to $\mathrm{pV}$ if the charge is a perfect gas). Since

$$
\mathrm{pV}=\mathrm{p}_{\mathrm{IVC}} \mathrm{V}\left(\frac{\mathrm{V}_{\mathrm{IVC}}}{\mathrm{V}}\right)^{\eta}
$$

where $\eta$ is the empirical power law exponent, the use of $\ln \left(\mathrm{V}_{\text {IVC }}\right)$ has the effect of linearizing the relationship. To retain consistency, all the other variables were similarly transformed. The additional advantage was that then all the quantities were dimensionless.

The targets were the Net Indicated Mean Effective Pressure (NIMEP) and the fuel equivalence ratio $\Phi$. Thus the desired target vector $\underline{r}$ was:

$$
\underline{r}=\left[\begin{array}{c}
\ln \left(\text { NIMEP }^{\prime} / \text { NIMEP }_{\text {ref }}\right) \\
\ln (\Phi)
\end{array}\right]
$$

The measurements were the cylinder pressure $p$, from which the NIMEP value was calculated in real time, and the exhaust fuel equivalence ratio. The exhaust lambda sensor had a response time of approximately $100 \mathrm{~ms}$ which corresponds to 1 engine cycles at $1200 \mathrm{rpm}$. The signal was not compensated for the response time so that the measured value may be considered as the averaged one over several cycles. Thus the measured vector $y$ of the target was:

$$
\underline{y}=\left[\begin{array}{c}
\ln \left(\text { NIMEP }_{\text {measured }} / \text { NIIMEP }_{\text {ref }}\right) \\
\ln \left(\Phi_{\text {measured }}\right)
\end{array}\right]
$$


It should be noted that the reference values, as long as they were consistently used in the engine calibration process, were completely immaterial. In our particular implementation, the values were $\mathrm{m}_{\mathrm{f}, \mathrm{ref}}=1 \mathrm{mg} ; \mathrm{V}_{\text {ref }}=1 \mathrm{~m}^{3} ; \mathrm{NIMEP}_{\text {ref }}=1 \mathrm{bar}$.

\section{Nature of HCCI engine dynamic behavior}

In the development process, the traditional state variable control approach was first used. The state variables, which encompassed the engine behavior, were chosen to be the mass of fuel $\left(\mathrm{m}_{\mathrm{f}}\right)$, air $\left(\mathrm{m}_{\mathrm{a}}\right)$, and residual $\left(\mathrm{m}_{\mathrm{r}}\right)$ in the charge, and the cylinder pressure $\left(\mathrm{P}_{-30}\right)$ at $30^{\circ}$ crank-angle BTC of the compression stroke. The latter value was chosen because it was a comprehensive representation of the thermal state of the charge before combustion. Thus the state vector $\underline{\mathrm{x}}$ was:

$$
\underline{x}_{i}=\left[\begin{array}{c}
m_{f} \\
m_{a} \\
m_{r} \\
p_{-30}
\end{array}\right]_{\text {cy clei }}
$$

An empirical engine model was used to estimate the state vector from the metered fuel, the exhaust lambda signal of the prior cycle, and the pressure trace. Note that value of the state vector for cycle $i$ was available at the beginning of cycle $i$. Then, since it was expected that the previous cycle should significantly impact the current cycle behavior, the engine state vector was modeled as a first order system

$$
\underline{x}_{i+1}=A \underline{x}_{i}+B \underline{u}_{i}+C
$$

where the coefficients $\mathrm{A}, \mathrm{B}$, and $\mathrm{C}$ represented the locally linearized engine behavior at a particular operating point. The values of these coefficients were to be identified through the transient response of the engine.

When the above procedure was applied to the engine, however, the relative contribution of the first term in Eq.(1.6) was found to be mostly negligible (much to our surprise). The reason was that the current cycle engine behavior depended on the previous cycle in a bipolar manner:

(a) The engine either responded quasi-statically, i.e. it responded instantaneously to the input - there was no cycle-to-cycle dynamics, or

(b) CAI combustion failed and the engine behave erratically (more information on that later).

Thus in case (a), the value of A in Eq.(1.6) was identified to be zero. In case (b), the engine behavior could not be represented by Eq. (1.6).

$\underline{\text { Controller design }}$

Based on the nature of the CAI engine transient behavior discussed in the last section, a CAI engine controller was designed to comprise the following parts:

(i) A ram rate limiter which ensured the targeted per cycle NIMEP increase was within the engine stability limit. 
(ii) A feed-forward controller, based on the static engine calibration table, which translated the targeted output vector $\underline{r}_{i+1}$ for the next cycle into the open-loop control vector $\underline{u}_{f, i}$. Note that since the CAI engine was essentially quasi-static, this feed-forward controller did not use the prior cycle information.

(iii)A feed-back control vector trim $\Delta \mathrm{u}_{\mathrm{i}}$, which was based on the cumulative prior cycle errors, to account for systematic calibration errors.

Since the engine calibration was done at a fixed intake condition and fuel, Part (iii) was necessary to correct for systematic changes such as variation in intake temperature, humidity and fuel type.

A schematic of the controller is shown in Fig. 1.10. Note that because the control variables for cycle $i+1$ had to be implemented at cycle $i$, at which only the measured output (NIMEP and $\Phi$ ) of cycle i -1 was available, the error term was based on the cumulative error up to cycle $\mathrm{i}$ -1 only. The feed-back part of the controller is defined by the following equations:

$\underline{\mathrm{w}}_{\mathrm{i}}=\underline{\mathrm{W}}_{\mathrm{i}-1}+\underline{\mathrm{e}}_{\mathrm{i}}$

$\underline{\mathrm{e}}_{\mathrm{i}}=\underline{\mathrm{r}}_{\mathrm{i}-1}-\mathrm{y}_{\mathrm{i}-1}$

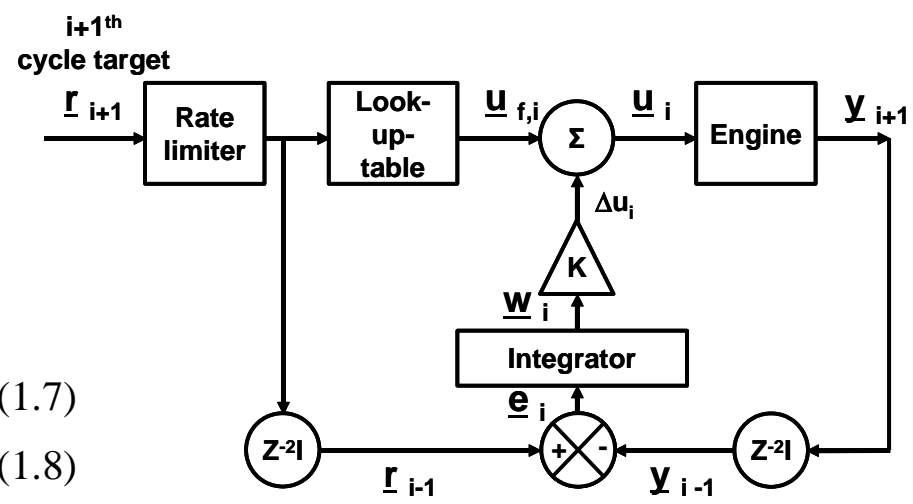

$\Delta \underline{\mathrm{u}}_{\mathrm{i}}=\mathrm{K}_{\underline{\mathrm{w}}}$

The feed-back gain matrix $\mathrm{K}$ may

(1.9.) 1.10 CAI closed-loop controller.

be designed to tailor the dynamic response of the overall system. (The dynamics were solely a result of the information cycle-lag of the feedback system.) We had only to determine $\mathrm{K}$ empirically through system simulation. According to Eqs. (1.7) and (1.9), the value of K may be interpreted as the trim coefficient; for example $\mathrm{K}_{11}=0.1$ implies that for a $10 \%$ cumulative positive error (measured value lower than targeted one) in NIMEP, the fuel mass has to increase by $1 \%$ in the next cycle. Note that the fuel trim had a higher gain than the exhaust valve timing trim, which in turn, had a higher gain than the intake valve timing trim. The same $\mathrm{K}$ matrix was applicable for all the operating points within the CAI region.

\section{Controller performance}

The engine behaviors in open-loop and closed-loop control were compared. In the first test, the targeted values were NIMEP $=4$ bar and $\Phi=1$. The intake air temperature was fixed at

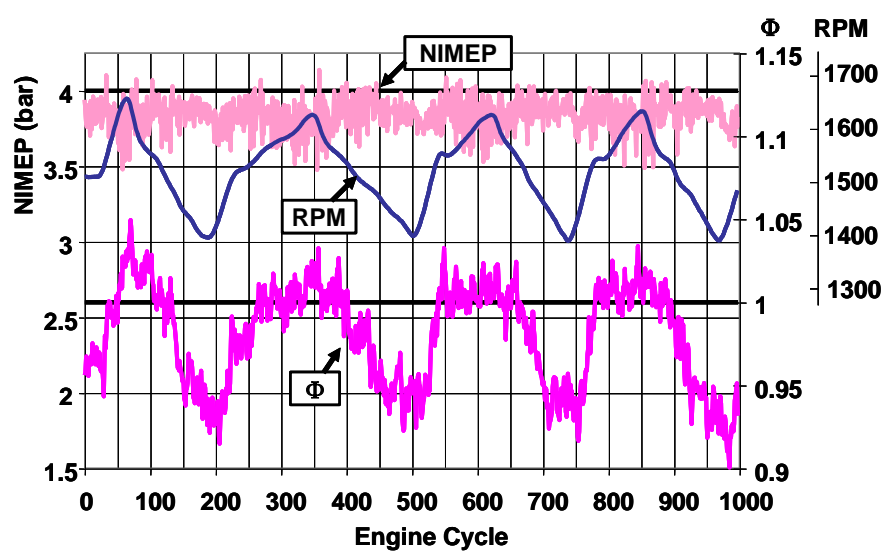

Fig. 1.11 Open-loop CAI engine behavior with respect to speed changes. Targeted values: NIMEP $=4$ bar; $\square=1$.

$120^{\circ} \mathrm{C}$. The engine speed was modulated between approximately 1400 to $1650 \mathrm{rpm}$ by hand- 
adjusting the dynamometer speed control setting. The open loop performance is shown in Fig. 1.11. There was a small but finite offset error in the NIMEP value, plausibly because the engine operating environment was different from that at calibration (e.g. the humidity had changed). There was a significant error on the $\Phi$ value and this error correlated with the speed modulation.

The error in $\Phi$ was traced back to a systematic error in the valve timing. Since an electromagnetic VVT system was used, the dynamics of the valve train were time based instead of crank-angle based. Therefore, there was a valve timing error when the speed was changed. This error, in turn, affected the gas exchange process so that there was an

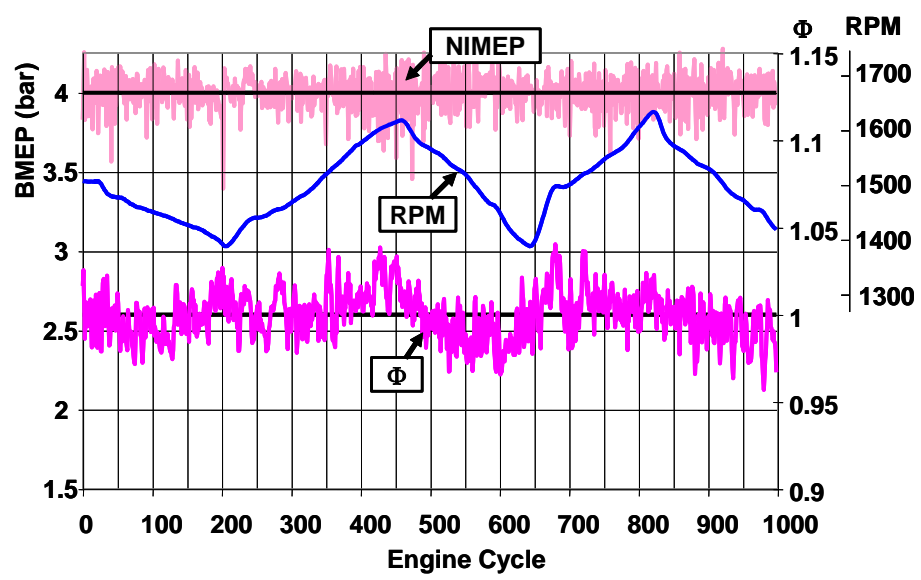

Fig. 1.12 Closed-loop CAI engine behavior with respect to speed changes. Targeted values: NIMEP $=4$ bar; $\Phi=1$. inducted air mass change, which resulted in the error in $\Phi$.

In the closed-loop controller, the NIMEP error was practically eliminated and the $\Phi$ error was substantially reduced; Fig. 1.12. When the control variables were examined, the valve timings were indeed adjusted by the controller to cancel the systematic timing error.

To substantially test the controller, the operating conditions were changed simultaneously and asynchronously. The temperature was modulated between 100 and $120^{\circ} \mathrm{C}$. The speed ws modulated from 1350 to $1650 \mathrm{rpm}$. The $\Phi$ target was at stoichiometric. The NIMEP target was changed in a sequence of steps with a transition time for each step equal to 0.25 second and then the NIMEP stayed constant for 3 seconds before the next transition.

The open-loop behavior of the engine in response to these changes is shown in Fig. 1.13. There were significant $\Phi$ errors which correlated with the speed modulation due to the systematic errors in the valve timing response as a function of engine speed. The NIMEP errors were also substantial.

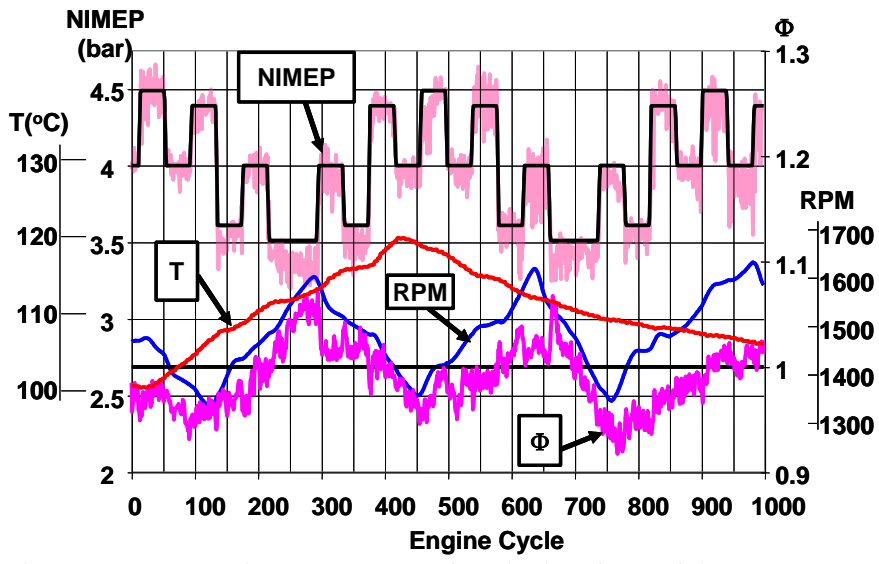

Fig. 1.13 Open-loop CAI engine behavior with respect to simultaneous variations in intake air temperature, engine speed and NIMEP target; $\Phi$ targeted at stoichiometric.

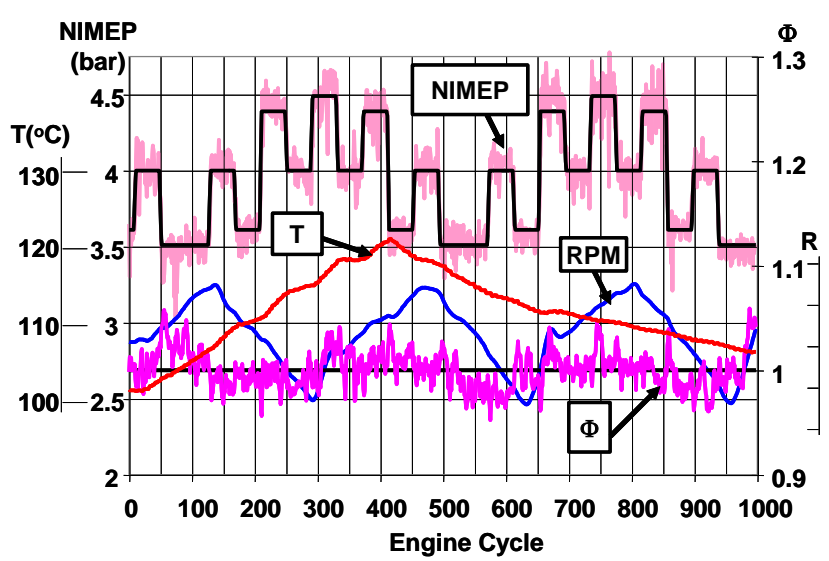

Fig. 1.14 Closed-loop CAI engine behavior with respect to simultaneous variations in intake air temperature, engine speed and NIMEP target; $\Phi$ targeted at stoichiometric. 
The closed-loop behavior of the engine under the same test is shown in Fig. 1.14. Both the NIMEP and $\Phi$ errors were substantially reduced. The error statistics are shown in Table 1.2. Comparing the closed-loop to openloop behavior, the mean NIMEP and $\Phi$ deviations from the target value were reduced by more than an order of magnitude. The reductions in the coefficient of variations were sizable but not as large (less than a factor of 2).
Table 1.2 Statistics of deviation from targeted values

\begin{tabular}{|l|l|l|l|l|}
\hline & \multicolumn{2}{|l|}{ Mean error } & \multicolumn{2}{l|}{$\begin{array}{l}\text { Coef. Of } \\
\text { Variations }\end{array}$} \\
\hline & NIMEP & $\Phi$ & NIMEP & $\Phi$ \\
\hline $\begin{array}{l}\text { Open- } \\
\text { loop }\end{array}$ & $-1.74 \%$ & $-1.05 \%$ & $3.2 \%$ & $3.1 \%$ \\
\hline $\begin{array}{l}\text { Closed- } \\
\text { loop }\end{array}$ & $-0.03 \%$ & $0.05 \%$ & $2.6 \%$ & $1.8 \%$ \\
\hline
\end{tabular}




\section{HCCI Engine Thermal Management under Transients (UM)}

An extensive study has been carried out on wall temperature and heat flux in an HCCI engine. The work involved the installation of 9 fast thermocouples in the UM HCCI engine. Two thermocouples were mounted in the head and seven on the piston crown. A telemetry linkage system was built to obtain the data on an instantaneous basis shown in Figure 1.15. This permitted the measurement of internal wall temperature and by a standard analysis, the instantaneous heat flux. Also, an intake fuel vaporizer was employed to operate under fully premixed operation. The engine is based on GM hardware, and features direct injection of gasoline, a compression ratio of 13 and a rebreathing exhaust camshaft. The engine test setup is shown in Fig. 1.16.

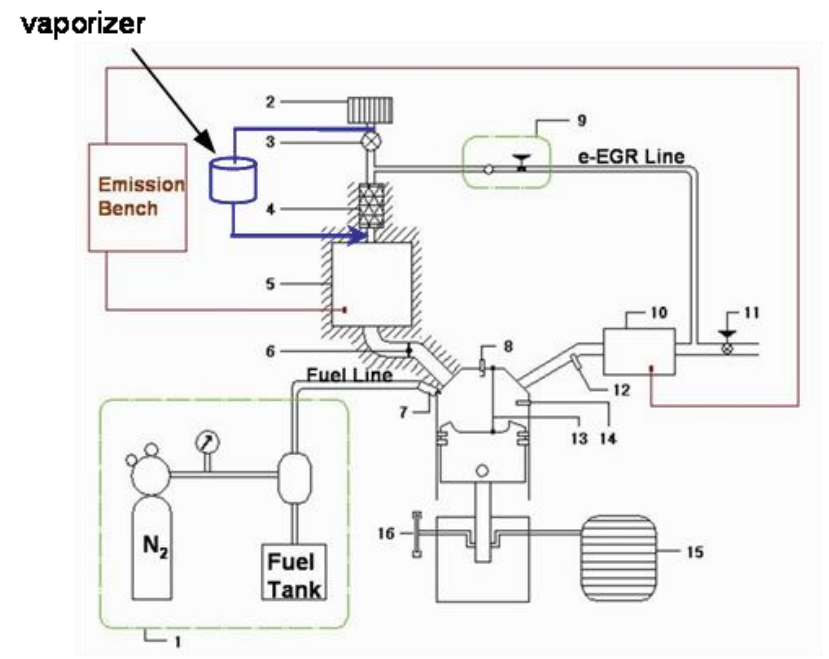

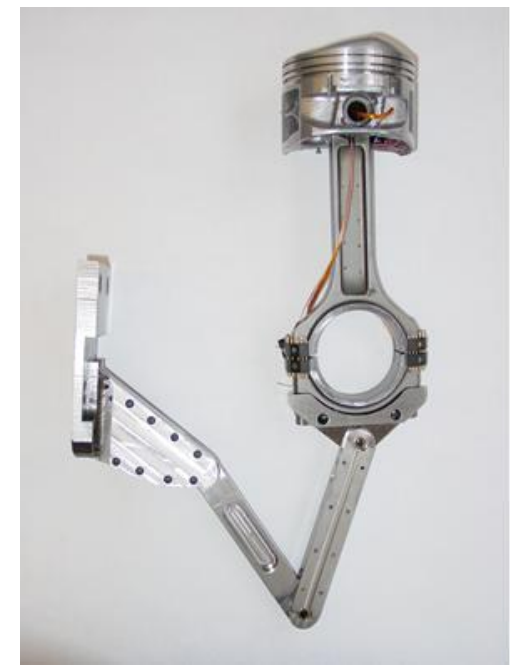

Fig. 1.15. Telemetry system for piston surface heat transfer probes in UM engine

Fig. 1.16. UM engine setup showing new fuel vaporizer.

\section{Heat transfer results}

Based on the results the average cylinder heat transfer coefficient was computed and compared to a number of well known heat transfer correlations. For these calculations, the cylinder average temperature was calculated based on a simple analysis of pressure and estimated cylinder mass. Figs. 1.17 and 1.18 show the results for heat transfer coefficient and heat flux respectively at $2000 \mathrm{rpm}$ and $\mathrm{A} / \mathrm{F}=20.3$. 
The solid black line indicates the measured average heat flux (Fig. 1.18) and the inferred heat transfer coefficient (Fig. 1.17) based on an estimated average gas temperature. Also shown are corresponding calculations from well known correlations, including the Woschni (1967)], Hohenberg (1979, 1982), Eichelberg (1939), and a modified Woschni model. The coefficient " $\mathrm{C}_{0}$ " shown in the figures is a multiplier applied to the overall expressions to achieve energy closure during the combustion part of the cycle.

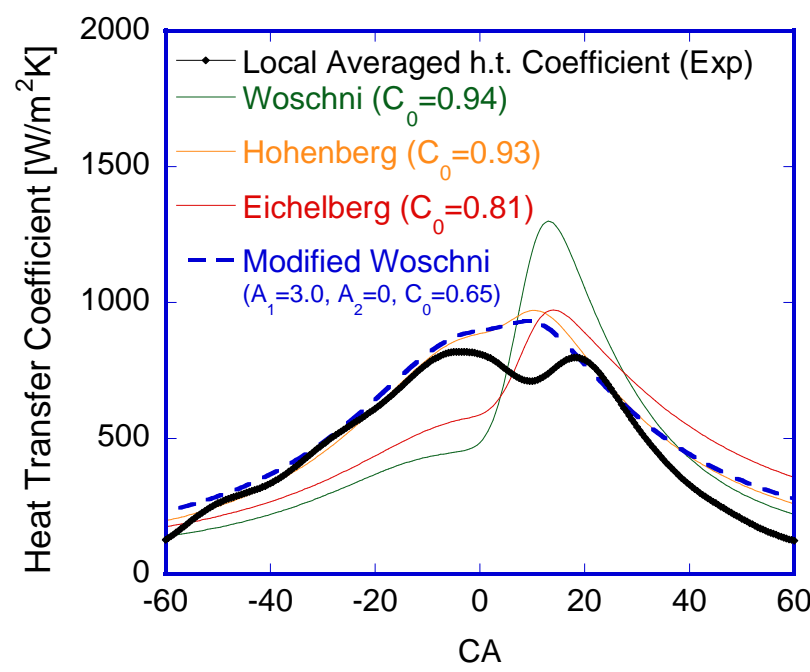

Fig. 1.17. Experimental heat transfer coefficient compared with models. Fully premixed operation; $2000 \mathrm{rpm}$; gasoline fuel $(\mathrm{A} / \mathrm{F}=20.3)$

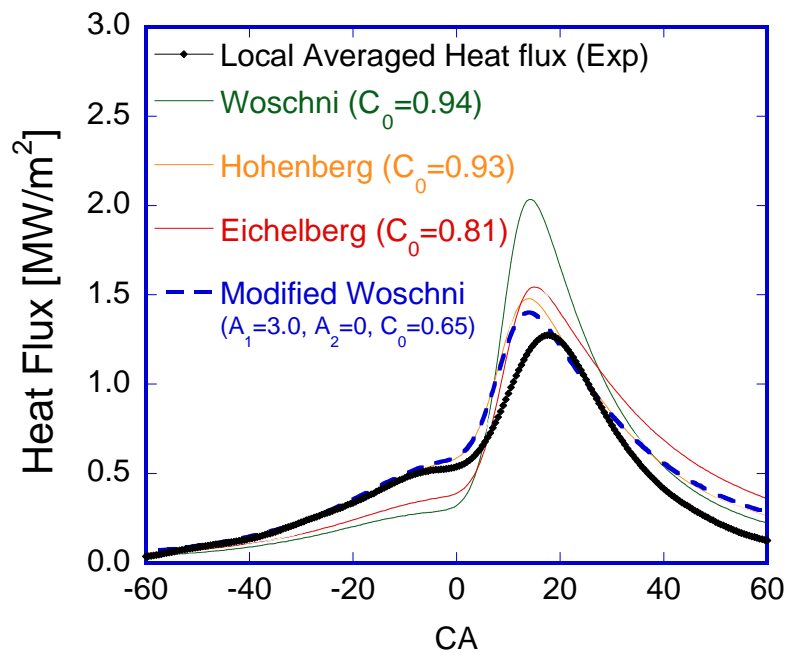

Fig. 1.18. Experimental heat flux compared with heat flux models. Fully premixed operation; $2000 \mathrm{rpm}$; gasoline fuel $(\mathrm{A} / \mathrm{F}=20.3)$

Of the three standard models, the Hohenberg seems the best, while the Woschni appears the worst. The Woschni model predicts a large peak during combustion, while the measured local average heat transfer data show only a moderate rise. We believe this is due to the provision in the Woschni model for the induced sloshing velocity during flame propagation, which does not occur in HCCI combustion. Accordingly, we have modified the Woschni model to eliminate this term, as shown below:

$$
h=C_{0} \cdot 3.26 \cdot B^{-0.2} \cdot p^{0.8} \cdot T^{-0.53} \cdot w^{0.8}
$$

where: $\quad w=A_{1} C_{1} \bar{S}_{p}+A_{2} C_{2} \frac{V_{d} T_{r}}{p_{r} V_{r}}\left(P-P_{m}\right)$

and:

$$
C_{1}=2.28+0.308 \frac{\pi B w_{p}}{\bar{S}_{p}} ; \quad C_{2}=0.00324
$$


$\bar{S}_{p}$ is the mean piston speed and $w_{p}$ is the angular swirl speed in rps. The two additional multipliers $A_{1}$ and $A_{2}$ are used to tune the shape of the curve, while $C_{0}$ is an overall level adjustment. Best results were obtained with $A_{1}=3.0$ and $A_{2}=0.0$, and overall $C_{0}=0.65$. (Note that the original Woschni model is recovered by setting $\mathrm{A}_{1}=\mathrm{A}_{2}=\mathrm{C}_{0}=1.0$.)

This result has been successfully implemented into the UM T-K cycle simulation model and the GT system model (Task 2) and provides confidence that the key heat transfer predictions will be as accurate as possible. For a full reporting see Chang, et al. (2004).

\section{Modeling and Experiments on HCCI Thermal Management under Transients (UM)}

A recent experimental study by Chang at al. (2005) with a rebreathing engine demonstrated a strong coupling between engine thermal conditions and the combustion phasing; i.e., the coolant temperature and the heat transfer through the walls play a great role in determining the combustion timing and phasing. While it is well known that the engine intake temperature is important for HCCI engine operation, this study showed that wall and coolant temperatures also have an equal or greater impact on the HCCI combustion as shown in Figure 1.19 .

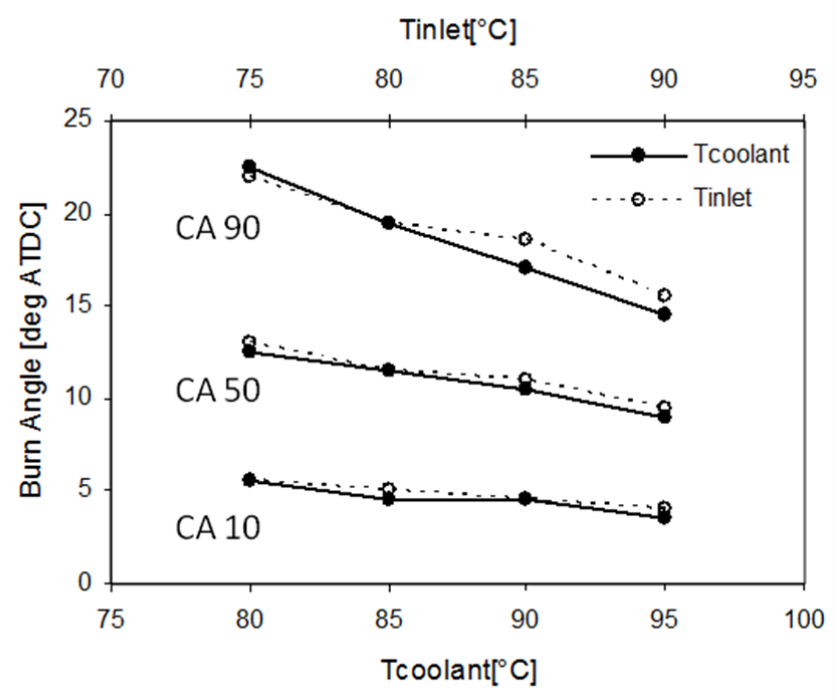

Figure 1.19. Experimental burn angles showing similar effects of coolant and inlet temperature variations

We analyzed these results using a simple model developed in Task 2 which integrates a custom combustion module into the GT-power system package, (Chang et al, 2006). This model is based on an auto-ignition expression taken from Task 3 and suitable correlations for burn rate and combustion efficiency drawn from UM engine data. It also incorporates the new heat 
transfer correlation specific to HCCI engines derived at UM described above. The model was able to successfully replicate the steady state wall temperature effect as shown in Figure 1.20 where model predictions of gas temperatures are compared with experimentally derived values over a range of coolant temperatures. For more details of the model see Task 2.
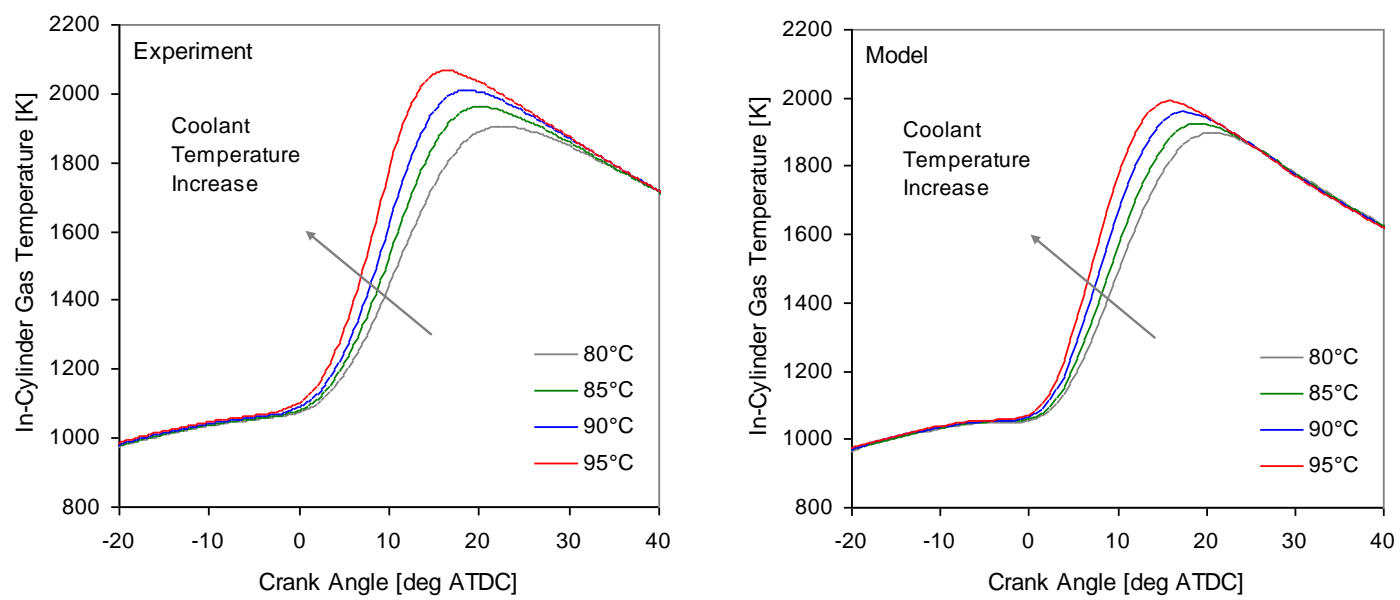

Figure 1.20. Cylinder gas temperature changes with varying coolant temperature. Left: temperatures inferred from experimental engine pressure; right: model predictions.

By means of this model we have explored some of the implications of "non-equilibrium" wall temperature in normal transient engine operation. This transient-like behavior arises due to the fact that the coolant temperature and wall temperature respond much slower than the actual speed and load conditions of the engine. This introduces a mismatch between steady state and "transient" conditions. This feature is important for calibration purposes because it makes the operating parameters path dependent over the speed load map.

To illustrate this effect, we considered the visitation map shown in Fig. 1.21 derived as part of Task 2 for a typical passenger vehicle over the FTP test procedure.

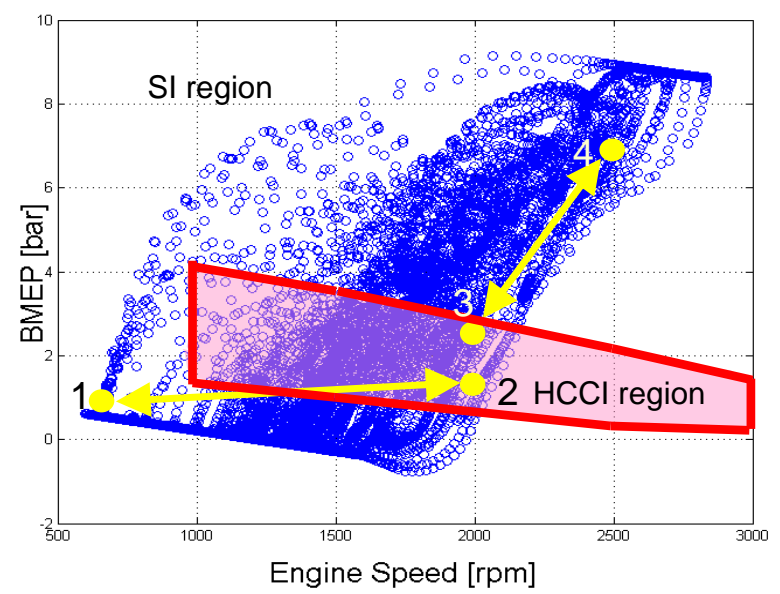

Fig. 1.21. Speed load visitation map for a typical SI engine vehicle during the FTP driving cycle; arrows show speed and load transitions modeled 
The four operating points shown in the figure and marked 1, 2, 3, and 4 denote idle, low load, mid load, and high load conditions respectively. In general the steady state wall temperatures will rise with overall power level, in the order 1 to 4 . Table 1.3 shows the steady state temperatures calculated at the four points by the GT-power finite element mesh submodel for average head, piston and cylinder liner temperature.

Table 1.3. Calculated steady state wall temperatures for the four points in Fig. 1.21.

\begin{tabular}{|l|l|l|l|l|l|l|l|}
\hline & RPM & $\begin{array}{l}\text { BMEP } \\
{[\mathrm{bar}]}\end{array}$ & $\begin{array}{l}\text { Fuel } \\
{[\mathrm{mg} / \mathrm{s}]}\end{array}$ & $\begin{array}{l}\text { Piston } \\
{[\mathrm{K}]}\end{array}$ & $\begin{array}{l}\text { Head } \\
{[\mathrm{K}]}\end{array}$ & $\begin{array}{l}\text { Cyl. } \\
{[\mathrm{K}]}\end{array}$ & $\begin{array}{l}\text { Avg } \\
{[\mathrm{K}]}\end{array}$ \\
\hline Point1 & 700 & 1.49 & 40 & 391 & 387 & 379 & 386 \\
\hline Point2 & 2,000 & 1.82 & 150 & 418 & 413 & 398 & 410 \\
\hline Point3 & 2,000 & 2.59 & 183 & 425 & 422 & 402 & 416 \\
\hline Point4 & 2,500 & 7.03 & 500 & 468 & 478 & 430 & 459 \\
\hline
\end{tabular}

Points 1 and 4 assumed SI operation with normal valve events, while points 2 and 3 were calculated using the autoignition module and a rebreathing exhaust cam for HCCI operation in the UM engine. The valve events for HCCI operation are shown in Fig. 1.22.

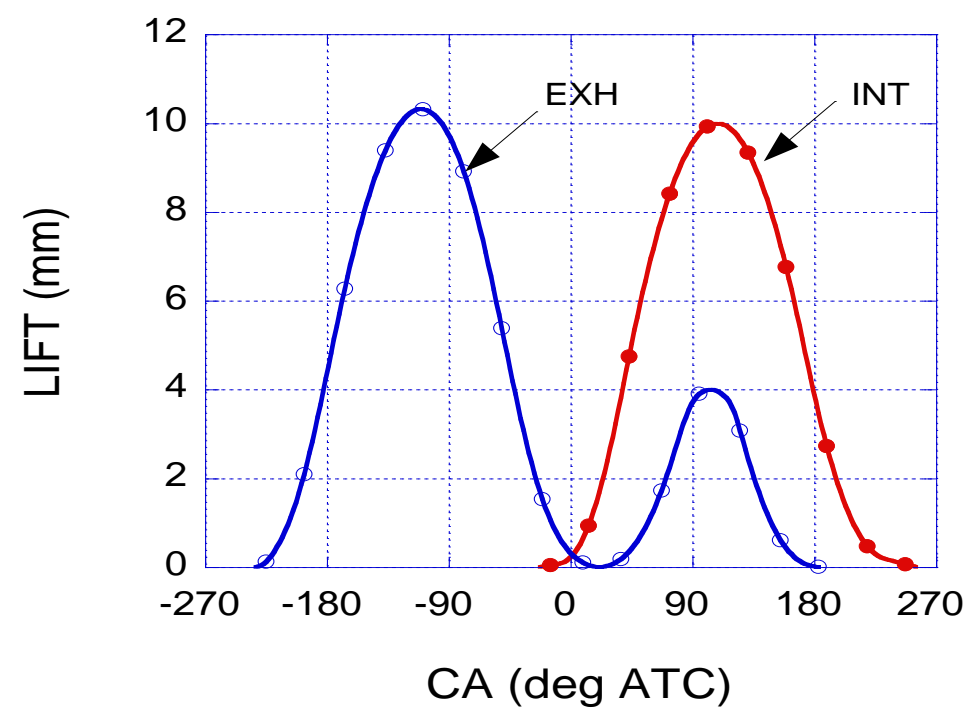

Fig. 1.22. Valve lift curves for HCCI operation showing rebreathing exhaust cam lift $=4 \mathrm{~mm}$.

We then considered two possible transition paths from SI to HCCI mode. The first was a deceleration from point 4 to point 3 , and the second was a tip-in from idle at point 1 to an offidle condition at point 2 . In each case, the engine operating conditions were changed to the final 
state at the start of the transition. For the purposes of obtaining the temperature history it was assumed that combustion always occurred near TDC. The average temperature history is shown in Fig. 1.23. The results show a gradual stabilization of temperature over a relatively long time period of 60 seconds or more corresponding to the thermal inertia of the metal parts of the head cylinder and piston.

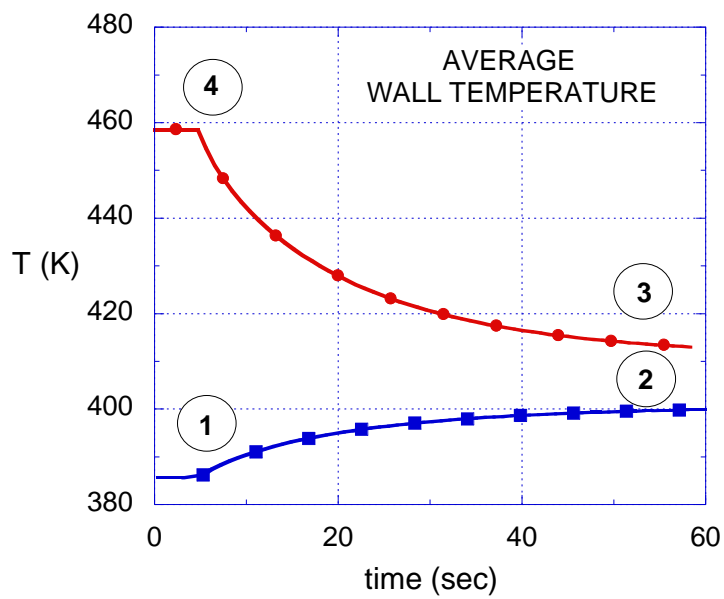

Fig. 1.23. Calculated wall temperatures during hot-cold (4-3), and cold-hot (1-2) transitions.

Next we ran the model in HCCI mode at the desired final speed and load, e.g. P3, and P2, but with the initial wall temperatures from point 4, denoted P3T4, and from point 1, denoted P2T1. (The steady state points are denoted by P3T3 and P2T2 respectively). The results are shown in Fig. 1.24 and demonstrate unacceptable behavior; in the case of the 4-3 hot-to-cold transition (P3T4), the charge is initially too hot and the combustion is too advanced, while for the 1-2 cold-to-hot transition (P2T1), the engine fails to fire.
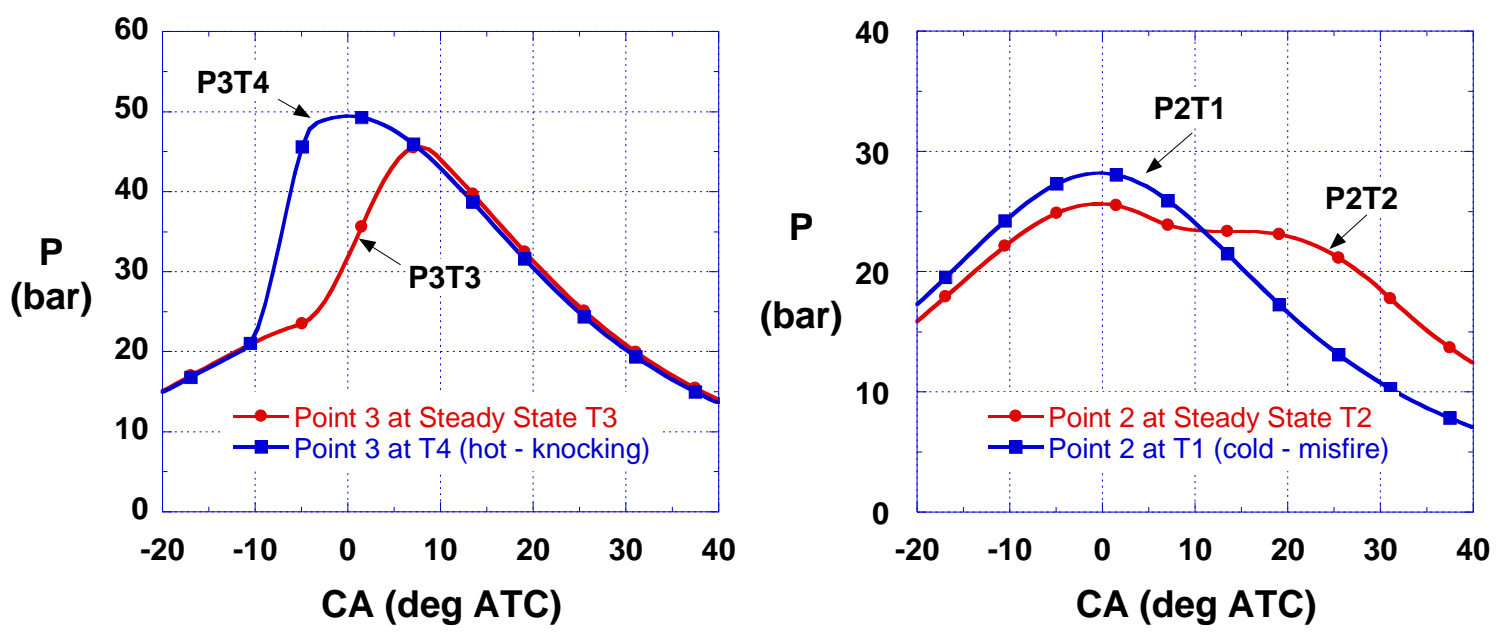

Fig. 1.24. Effect of non-steady state wall temperature on performance at two HCCI points. Left: initially hot; right: initially cold. 
We explored whether these transients could be satisfactorily managed by dynamically changing the amount of internal EGR by varying the rebreathing lift. Fig. 1.25 shows the results on Start of Combustion (SOC), EGR \%, and BMEP for the points P3T3 and P3T4.
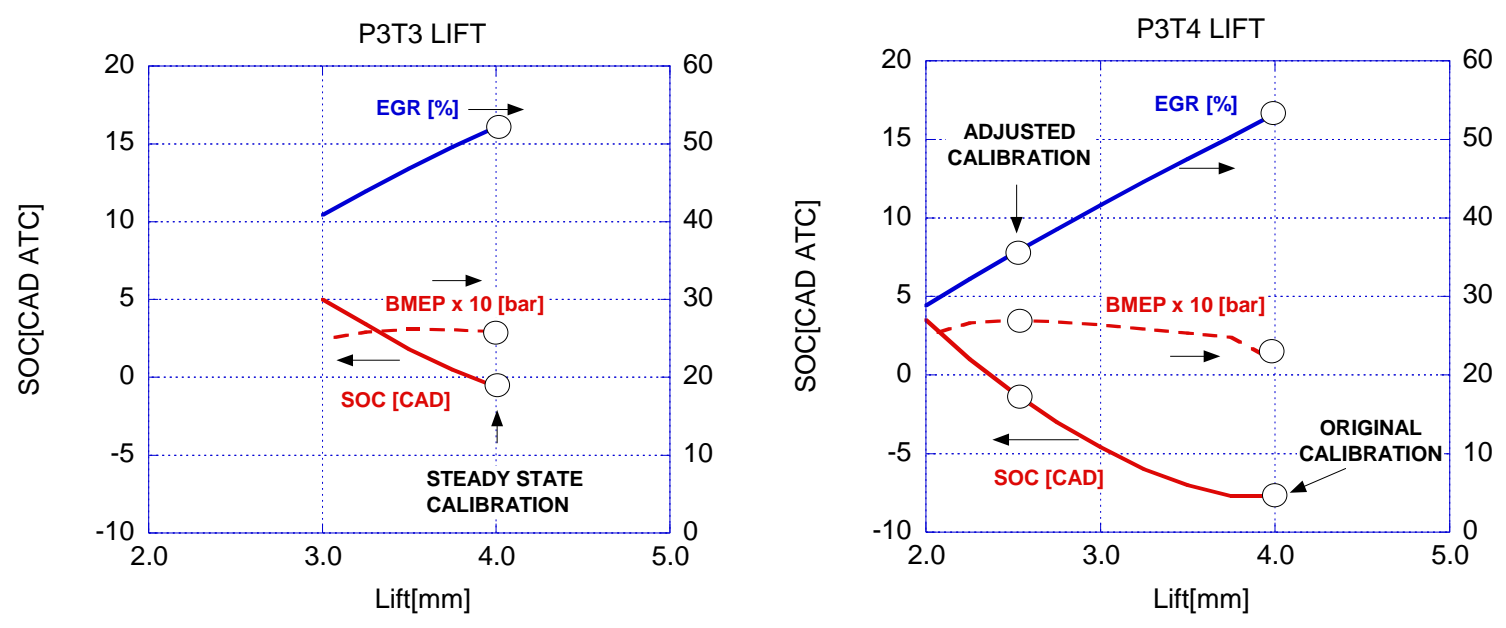

Fig. 1.25. The effect of rebreathing valve lift on the calculated SOC, EGR\% and bmep at P3T3 and P3T4. Original and adjusted calibration points are shown by the open circles.

As the lift is increased the level of internal EGR increases, and the temperature of the charge increases and the SOC advances. For all the runs shown the amount of fuel in the cylinder was fixed to approximate constant load operation. Because of this, when EGR is increased, the fresh air decreases and the A/F falls. Above about 52\% EGR, the A/F ratio falls below stoichiometric and the burn rate correlation is no longer valid. Rich operation is undesirable anyway because of the $\mathrm{CO}$ emissions and efficiency fall off. For this reason the curves show only the lean region with lift $\leq 4.0 \mathrm{~mm}$.

The left side of Fig. 1.25 shows the steady state calibration for P3 at T3. (4.0 mm lift, Pin $=0.95$ bar). This gives a good SOC of just before TDC. The right side of Fig. 1.25 shows the unsteady state point of $\mathrm{P} 3$ at the hotter T4. The original $4 \mathrm{~mm}$ calibration is shown at the right side of the figure, and gives an overly advanced SOC of $-7.5^{\circ}$ ATC. If the lift is reduced to 2.5 $\mathrm{mm}$ the EGR is reduced and SOC is retarded to a more satisfactory $-1.5^{\circ}$ ATC which also has a higher BMEP. 


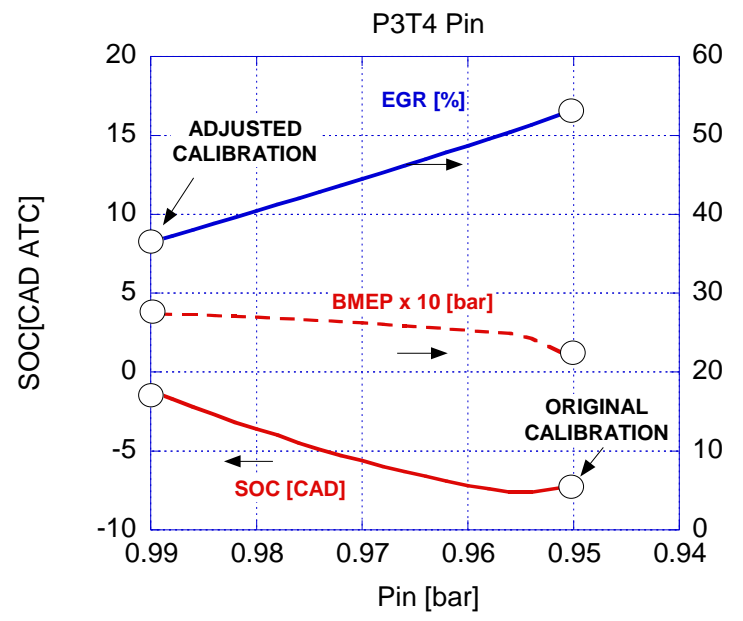

Fig. 1.26. The effect of manifold pressure variation on the calculated SOC, EGR\% and bmep at P3T3 and P3T4. Pexh $=1.05$ bar. Original and adjusted calibration points are shown by the open circles.

With a rebreathing cam, small changes in manifold pressure can cause relatively large changes in residual gas fraction. With this in mind we made a second set of calculations varying the manifold pressure from 0.95 to 0.99 bar with exhaust pressure fixed at 1.05 bar. Results shown in Fig 1.26. are similar to those shown in Fig. 1.25 and indicate that at higher manifold pressure (0.99 bar) the EGR is reduced and combustion is retarded to a satisfactory crank angle.

Fig. 1.27 shows the corresponding pressure curves. Satisfactory adjusted pressure curves are obtained by the strategy of reducing the lift or by raising the manifold pressure slightly. This suggests that the hot-to-cold transient can be managed by a rapid shift in either the lift or manifold pressure and then slowly reducing the shift to compensate for the wall temperature as it relaxes to steady state.

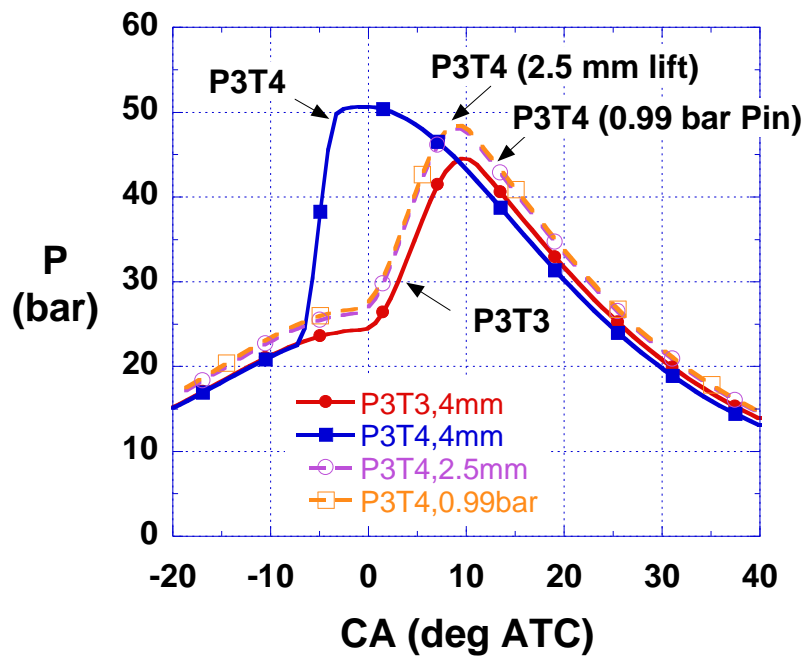

Fig. 1.27. Calculated HCCI pressure traces for point 3. Shown are the steady state case P3T3 and the transient early burning case P3T4, both with $4 \mathrm{~mm}$ lift, along with the adjusted cases with reduced lift $(2.5 \mathrm{~mm})$ and increased manifold pressure ( 0.99 bar) to retard the combustion. 
In the cold-to-hot case, P2T1, we were unable to get combustion regardless of the lift. Increasing the lift (and residual) was not sufficient to induce ignition in part because the steady state case was on the edge of the stable combustion regime. Other means of thermal management would be required to expand the range.

To explore the more general case, a more systematic study was carried out in which an optimal RGF rate was calculated over a wide range of the speed load map for three different initial conditions - hot, cold and medium wall temperatures. For some conditions HCCI combustion was not possible either because of misfire (ignition failure) or because of knock (combustion was too rapid). The results are shown in Figures 1.28.
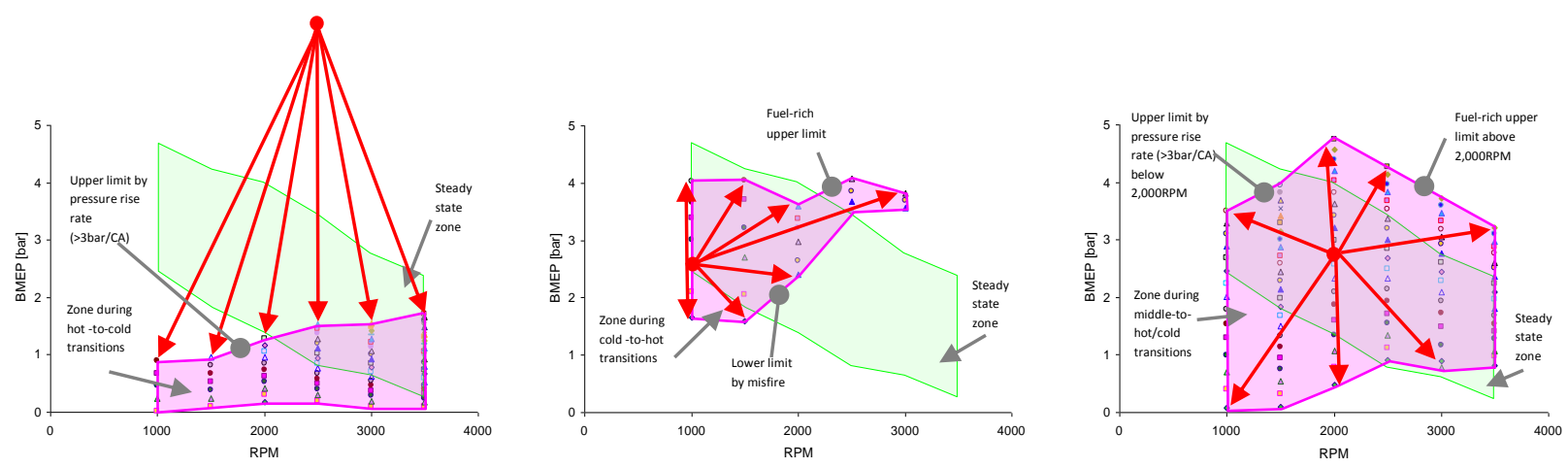

Figure 1.28. Shifted HCCI operating zone during engine transitions from three initial thermal conditions: a) hot, b) cold, c) mid range. Green indicates steady state stable HCCI range while pink indicates the transient stable range.

The stable range of HCCI operation is significantly affected by the initial condition and that in some cases the range is actually expanded from steady state. Within the steady state region and for hot-to-cold transitions this effect can be reduced or eliminated by changing the residual fraction on the same time scale as the thermal transient, either by valve lift changes or by small changes in inlet pressure. In the case of cold-to-hot transitions, this is not always possible. It will then be necessary to temporarily extend the SI mode well into the steady state HCCI regime, until the wall temperature increases. In the case of hot-to-cold transitions it may also be possible to extend the HCCI mode into low load regions not normally accessible under steady state conditions. Given the transient nature of normal driving cycles, this may be a substantial fraction of the time and may compensate for any losses experienced in cold-to-hot transitions. A full investigation into the fuel economy implications of this effect has not yet been carried out. 


\section{Multi-cylinder Engine Control (UCB)}

\section{Numerical and Experimental investigation of ion current sensor}

Ion sensors are an attractive potential combustion timing sensor for use in HCCI control systems. To investigate this possibility experiments were carried out as well as an analytical study modeling the production of ions in the combustion gases. A typical experimental result demonstrating the existence of ion current in an HCCI engine is shown in Figure 1.29. The ion signal can be seen to capture the Start of Combustion (SOC) as well as the pressure transducer. The ion sensor is substantially less expensive than pressure transducers used in research engines and it has been shown in the previous reports that the ion sensors work as well as pressure transducers. The experimental results are taken on a VW TDI 1.9 liter 4 Cylinder diesel engine converted to run in HCCI mode. The ion sensor is installed in the glow plug hole of the engine. The ion sensor circuit is shown in figure 1.30.

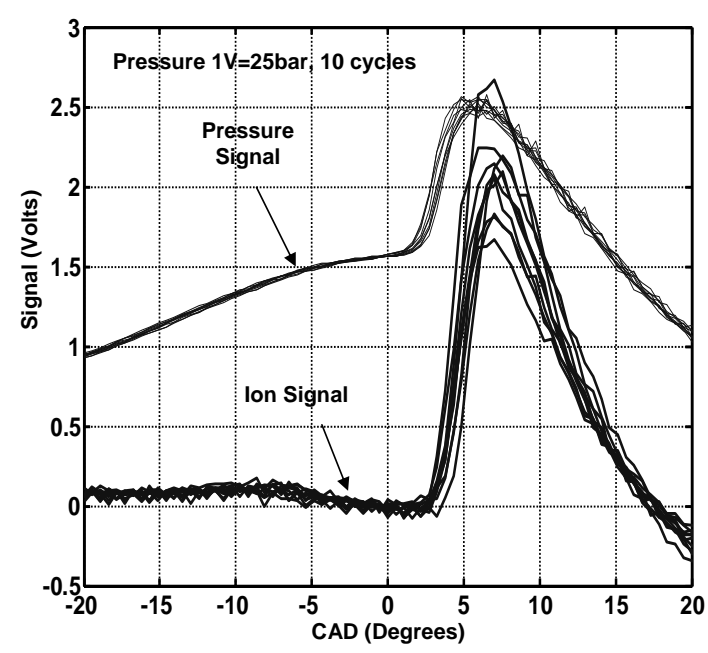

Figure 1.29. Ion signal (volts*100) and pressure transducer signal $(1 \mathrm{~V}=25 \mathrm{bar})$ for 10 consecutive cycles, $\Phi=0.38$.

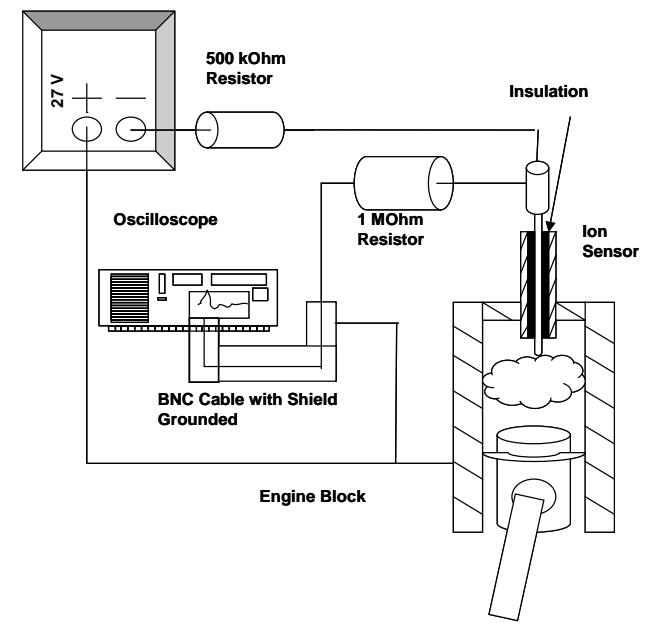

Figure 1.30. Schematic of an ion sensor in an HCCI engine. 
Along with the experimental investigations, a single zone model with detailed chemistry of ion generation was utilized to numerically investigate the process of ion generation and origins of ion in HCCI combustion. Efforts were directed to use the insight provided by the numerical model to improve the working range of ion sensor. A detailed discussion of our ionization model is given in previous reports and in the $30^{\text {th }}$ International Combustion Symposium (Mehresh et al., 2004). In brief, the ionization model contains 34 reactions involving 9 ionic species $\left(\mathrm{NO}^{+}, \mathrm{N}^{+}, \mathrm{N}_{2}{ }^{+}, \mathrm{O}_{2}{ }^{+}, \mathrm{OH}^{+}, \mathrm{O}^{+}, \mathrm{H}_{3} \mathrm{O}^{+}, \mathrm{HCO}^{+}\right.$, and electron). By far, the 5 most dominant reactions are presented in Table I. These reactions focus on the formation and consumption of $\mathrm{H}_{3} \mathrm{O}^{+}$as the main ion species. The initiation reaction for ionization is reaction (R1) in Table 1.4, which leads to the formation of $\mathrm{CHO}^{+}$. Here, the rapid formation of $\mathrm{H}_{3} \mathrm{O}^{+}$in the $\mathrm{CHO}^{+}$reaction with water (R3) is considered as well as $\mathrm{H}_{3} \mathrm{O}^{+}$consumption in the neutralization reactions (R4, R5). Furthermore, reaction (R2) accounts for the loss of $\mathrm{CHO}^{+}$in the neutralization reaction with electrons

Table 1.4. Rate Coefficients of major ionization reactions.

\begin{tabular}{|l|l|l|l|l|}
\hline & Reaction & $\mathrm{A}$ & $\beta$ & $\mathrm{E}_{\mathrm{a}}$ \\
\hline 1 & $\mathrm{CH}+\mathrm{O}->\mathrm{CHO}^{+}+\mathrm{e}^{-}$ & $4.600 \mathrm{E}+08$ & 0.73 & -2.6 \\
\hline 2 & $\mathrm{CHO}^{+}+\mathrm{e}^{-}-\mathrm{CO}+\mathrm{H}$ & $1.325 \mathrm{E}+17$ & 0.00 & 0.0 \\
\hline 3 & $\mathrm{CHO}^{+}+\mathrm{H} 2 \mathrm{O}->\mathrm{H} 3 \mathrm{O}^{+}$ & $1.000 \mathrm{E}+16$ & -0.09 & 0.0 \\
\hline 4 & $\mathrm{CO}^{\mathrm{H} 3 \mathrm{O}^{+}+\mathrm{e}^{-}->\mathrm{H} 2 \mathrm{O}+}$ & $2.291 \mathrm{E}+18$ & -0.50 & 0.0 \\
\hline & $\mathrm{H}$ & & & \\
\hline 5 & $\mathrm{H} 3 \mathrm{O}^{+}+\mathrm{e}^{-}-\mathrm{OH}+\mathrm{H}$ & $7.949 \mathrm{E}+21$ & -1.37 & 0.0 \\
\hline & & & & \\
\hline
\end{tabular}

The numerical model trends agreed well with the experimental trends. Figure 1.31 shows the dependence of the experimental ion-signal on the equivalence ratio for propane. The ion signal increases rapidly with increasing equivalence ratios. At $\Phi \sim 0.32$ for propane, the measured ion signal becomes unusable as the signal-to-noise ratio approaches one. This lower value of equivalence ratio is expected to be a function of the fuel used. The main ion generation reaction is the chemi-ionization reaction which is initiated by the presence of $\mathrm{CH}$ radicals. Hence, the fuels that produce large amount of $\mathrm{CH}$ radicals should produce higher ion concentrations and a better ion signal.

We tested this hypothesis using acetylene as fuel for another set of experimental results. The results are plotted in Fig.1.32. The reason for choosing acetylene as fuel is that acetylene produces more $\mathrm{CH}$ radicals compared to propane and most of the other hydrocarbon fuels. As evident from figure 1.32 , the ion signal is indeed strong at much lower equivalence ratio $(\sim 0.22)$ 
as compared to propane $(\sim 0.32)$. Hence we can safely say that acetylene does produce higher concentrations of ion which in turn gives stronger ion signal.

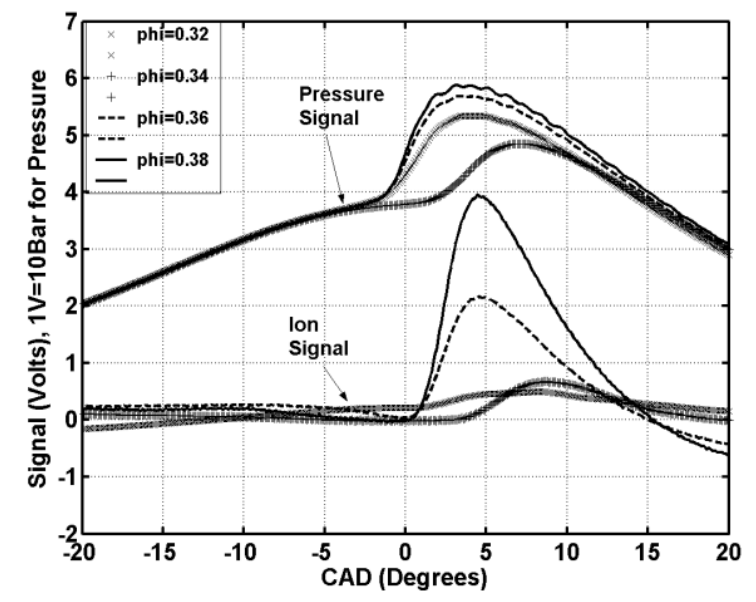

Figure 1.31. Experimental results showing a strong dependence of ion-signal on the equivalence ratio. Propane is used as the fuel for the above experiments.

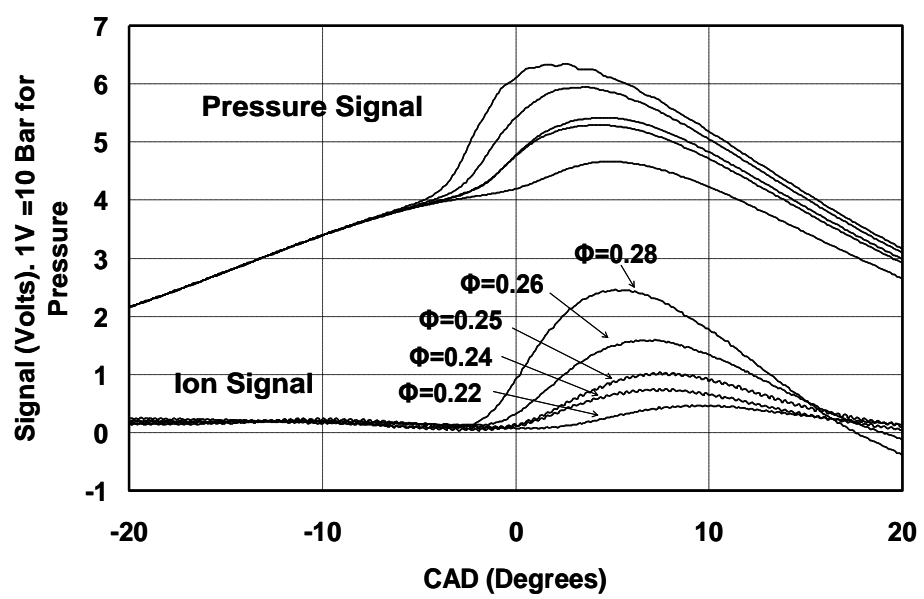

Figure 1.32. Experimental results show a strong dependence of ion-signal on the equivalence ratio. Acetylene is used as the fuel for the above experiments. Notice that ion signal is strong for low equivalence ratio $(\sim 0.22)$ unlike the propane results $(\sim 0.32)$.

The experimental investigation is further continued using various mixtures of acetylenein-propane. The acetylene mass fraction is varied from $0 \%$ to $40 \%$ in the mixture of acetylene and propane. The ion signal is measured for each mixture at similar conditions of temperature and pressure. Care is taken to keep the peak cycle temperature within $50 \mathrm{~K}$ for each run. This minimizes the effect of temperature on ion signal. The results are plotted in figure 1.33. It is seen in the figure that the ion signal become stronger as the acetylene mass fraction is increased in the acetylene propane mixture as a fuel.

A numerical model simulates the condition at the peak of combustion event. The model assumes constant temperature and constant pressure at values similar to the values measured in 
experiments. Chemical kinetic relations based on detailed propane and acetylene mechanism augmented with ionization model, are solved estimating species generation at the peak of combustion event. Figure 1.34 shows the estimated $\mathrm{CH}$ radical concentration at the peak of combustion at various conditions similar to the measured conditions in experiments. As seen from the figure, the $\mathrm{CH}$ radical concentration increases rapidly with increase in acetylene mass fraction in acetylene-in-propane mixture. This confirms that fuels producing more $\mathrm{CH}$ radicals produce higher concentration of ions and hence better ion signal. Additives which produce more $\mathrm{CH}$ radicals then help enhancing the ion signal and thus improve the usability of an ion sensor.

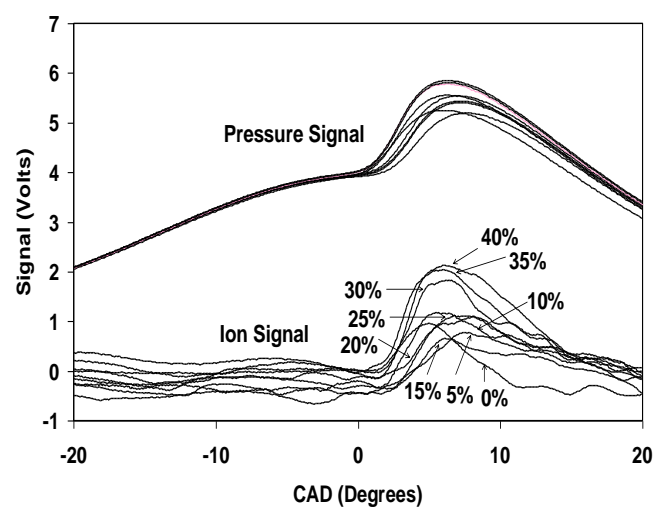

Figure 1.33. The ion signal become stronger as the acetylene mass fraction is increased in the fuel.

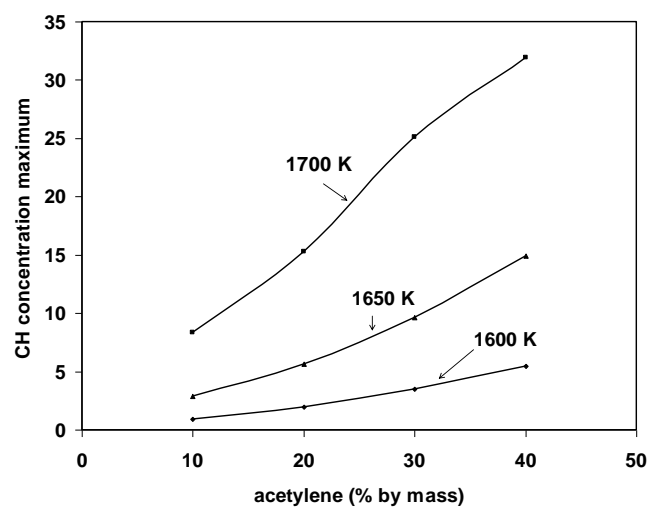

Figure 1.34. Maximum CH radical concentration (unit as mole fraction X 1011) increases rapidly with increase in acetylene mass fraction. Constant temperature, constant pressure model $(\mathrm{P}=60$ atm) results. The increase in $\mathrm{CH}$ radical concentration causes increase in ion concentration as seen in Figure 1.3.5.

\section{Multi-Cylinder HCCI model}

Another major focus of this project was a multi-cylinder HCCI model developed for use in the control design process. The model has been created in Simulink. The model was built upon 
the conservation of mass, conservation of energy, convective heat transfer, and compressible fluid flow through an orifice laws.

The model builds upon results from other members in the consortium. The start-ofcombustion model is based on the Livengood-Wu integral. The ignition delay, $\tau$, used in the Livengood-Wu integral is calculated using an Arhennius-type relation. The relation was generated by Professor J-Y. Chen and is based on the results of well-mixed reactor simulations. The heat transfer model used, a modified version of the Woschni model, was taken from the UM heat transfer work (above).

The multi-cylinder model includes an intake manifold mixing model, an exhaust manifold model, and models of the exhaust throttles. The model is comprehensive enough to capture the cylinder coupling effects seen in experiments, yet simple enough for the rapid simulations required by the control design process. Although the model could be used for controller synthesis, the model is most useful as a starting point for generating a reduced-order model, or as a plant model for evaluating potential controllers. Specifically, the model includes the dynamics for affecting the combustion timing through exhaust throttling.

A four-cylinder VW 1.9L TDI engine converted to run in HCCI mode is used for model validation. On the single cylinder Caterpillar $3401 \mathrm{HCCI}$ engine used in previous experiments, closing a throttle valve mounted in the exhaust stream increases the in-cylinder residual gases and thus the temperature when the intake valve closes, resulting in advanced combustion timing. On the VW engine, however, closing an exhaust throttle affects the combustion timing on two cylinders. These results are shown in Figure 1.35, where the exhaust throttle is closed on cylinder 3 just before $t=15$ seconds. The combustion timing on cylinder 3 advances by approximately 2 CAD. The combustion timing on cylinder 1 advances by approximately 1 CAD. A similar trend occurs when the exhaust throttles on the other cylinders are closed. Closing the exhaust throttle on a cylinder $z$ advances the combustion timing on cylinder $z$ as well as the cylinder that precedes cylinder $z$ in the firing order.

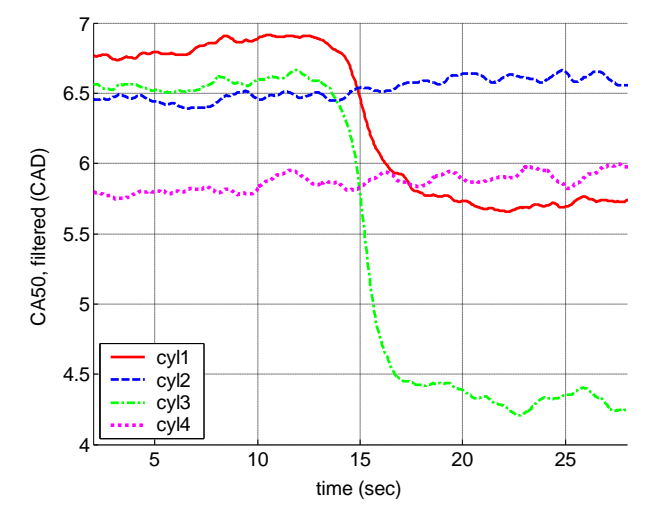

Figure 1.35. Experimental results showing that closing of an exhaust throttle on cylinder 3 advanced combustion timing on cylinder 1 and cylinder 3 . 


\section{Multi-variable Controller Design (UCB)}

In the course of the engine work cylinder-to-cylinder cross-talk was discovered and documented. Briefly cross talk occurs when an exhaust throttlet that is used to control residual gas in one cylinder, affects a neighboring cylinder because of manifold pressure dynamics. In this quarter, the multi-cylinder combustion timing control problem was addressed in detail on the VW 1.9L HCCI engine. A comprehensive model was developed to explore cross-talk and its implications on multi-cylinder control. Because of cross-talk, the single-cylinder closed-loop PID controller previously used cannot be directly extended to the multi-cylinder HCCI engine.

A $7^{\text {th }}$-order linear time-invariant (LTI) model was developed using subspace system identification. The model is of the form:

$$
\begin{aligned}
& x_{k+1}=A x_{k}+B u_{k}+K e_{k} \\
& y_{k}=C x_{k}+e_{k}
\end{aligned}
$$

A linear quadratic Gaussian (LQG) optimal CA50 tracking controller was designed. The LQG controller performed poorly on the multi-cylinder HCCI engine due to slowly-varying engine and cylinder wall temperature disturbances. Integral action was added to the LQG controller and the performance improves significantly. The resulting 11-state controller is not included here because of its complexity. The simulation results in Fig. 1.36 show a significant improvement in performance over the standard LQG controller. There is a small overshoot when the controller is first turned on, but the combustion timing quickly converges to the desired value.

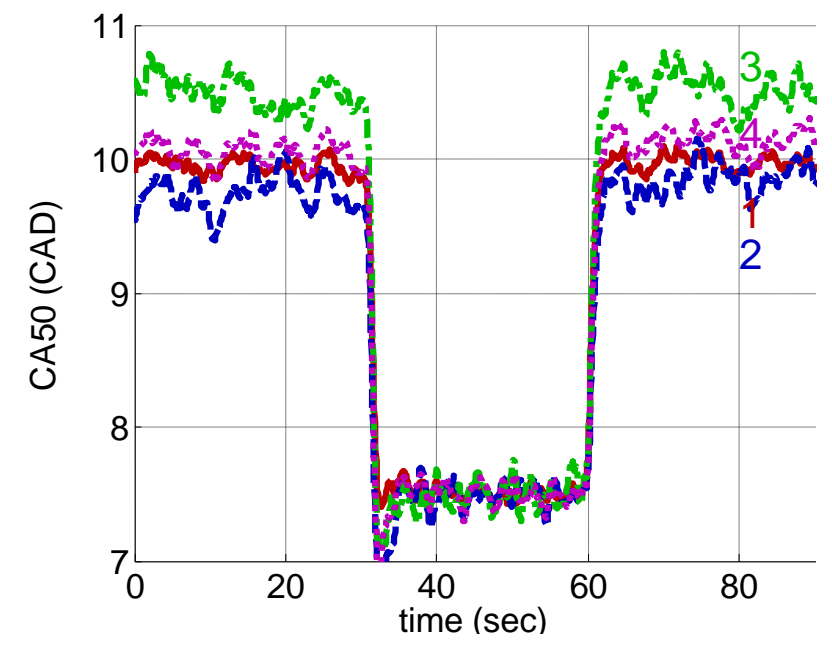

Figure 1.36. Simulation Results: LQG-Integral Controller Response, Plant Input Disturbance. Open-Loop 0-30, 60-90 sec. Desired CA50: 8 CAD 30-60 sec. (Adapted from Souder, 2004)

Figure 1.37 shows the equivalent experimental results. When the controller is turned on at $\mathrm{t}=30$ seconds, the combustion timing is balanced among all the cylinders and the tracking is excellent. Figure 1.4.2 shows another important feature of the multi-cylinder HCCI engine controller. The ' $x$ ' denotes misfires. Cylinders 2 and 3 are misfiring often when the engine 
controller is off, yet cylinders 1 and 4 fire regularly. The misfire limit for a particular cylinder depends on many factors: engine geometry, compression ratio, and coolant flow. Thus, each cylinder of a multi-cylinder HCCI engine behaves differently and controlling each cylinder independently is justified.

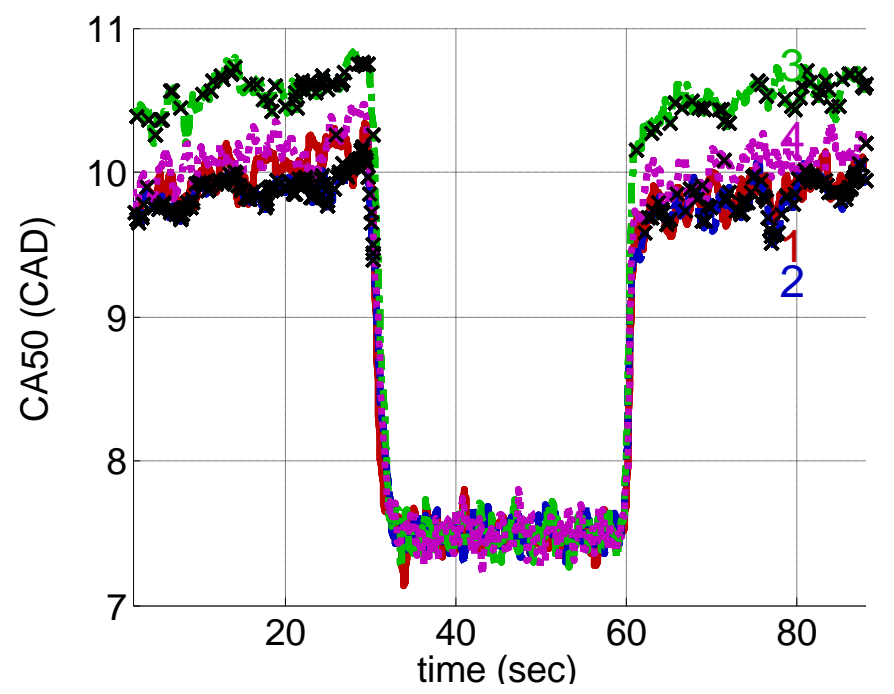

Figure 1.37. Experimental Results: LQG-Integral Controller Response, Plant Input Disturbance. Open-Loop 0-30, 60-90 sec. Desired CA50: 8 CAD 30-60 sec. ' $x$ ' Denotes a Misfire. (Adapted from Souder, 2004)

Figure 1.38 shows the LQG-integral controller's step response. The response and tracking are excellent. Figure 1.39 shows the control input for this experiment.

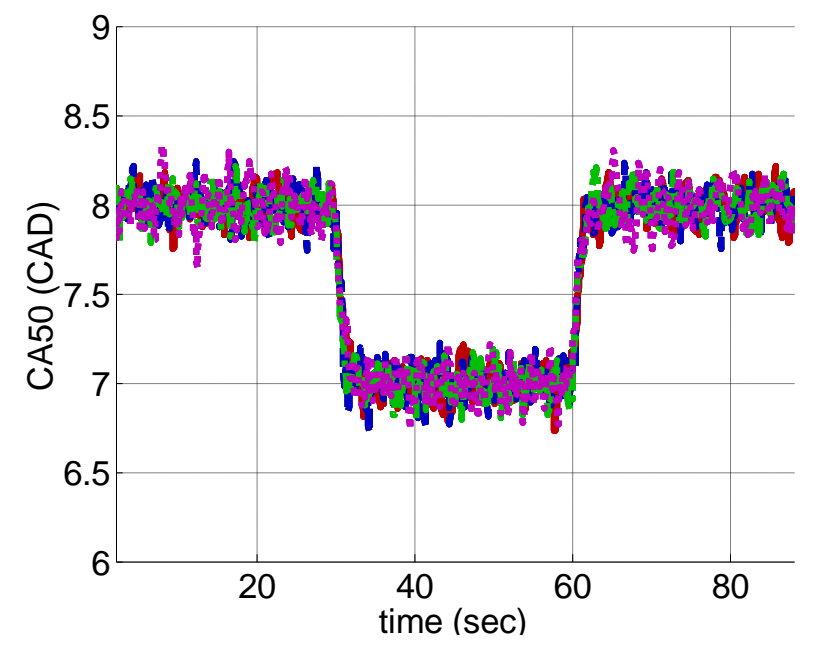

Figure 1.38. Experimental Results: LQG-Integral Controller Step Response, Desired CA50: 8 CAD 0-30, 60-90 sec; 7 CAD 30-60 sec. (Adapted from Souder, 2004) 


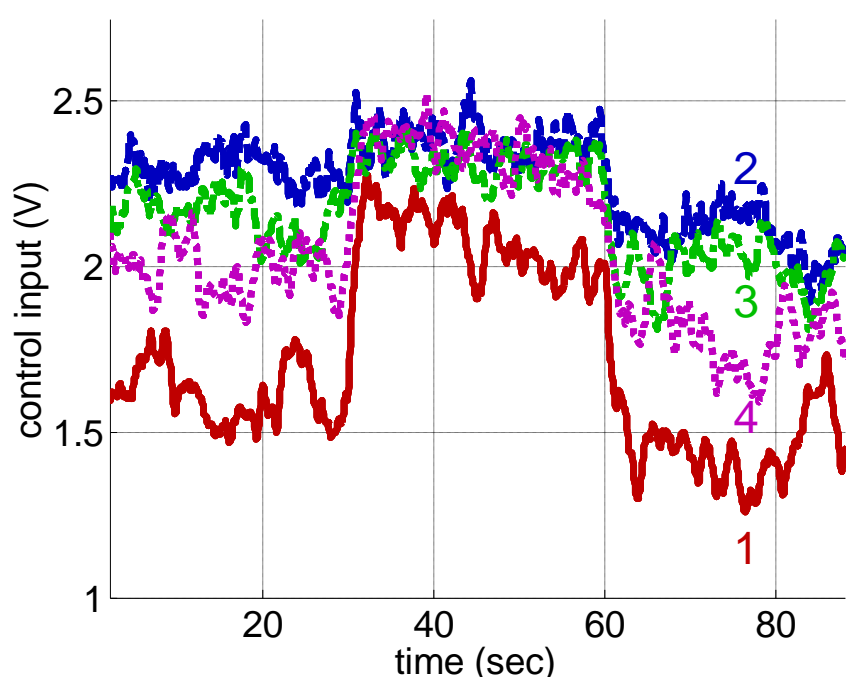

Figure 1.39. Experimental Results: Control Input for LQG-Integral Controller Step Response. (Adapted from Souder, 2004)

Figures 1.40 and 1.41 show that arbitrary combustion timing is possible. The combustion timing on each cylinder is set independently. At $t=30$ seconds, the cylinders are balanced. At $\mathrm{t}=60$ seconds, the desired combustion timings return to their initial values. Although such arbitrary combustion timing may not be desirable, the plots illustrate an important aspect of the MIMO HCCI controller: it is able to control the combustion timing on each cylinder independently via exhaust residuals, in spite of cylinder-to-cylinder cross-talk. These results also confirm that the slowly-varying engine and cylinder wall temperature effects can be treated as constant unknown disturbances and rejected by a controller with integral action.

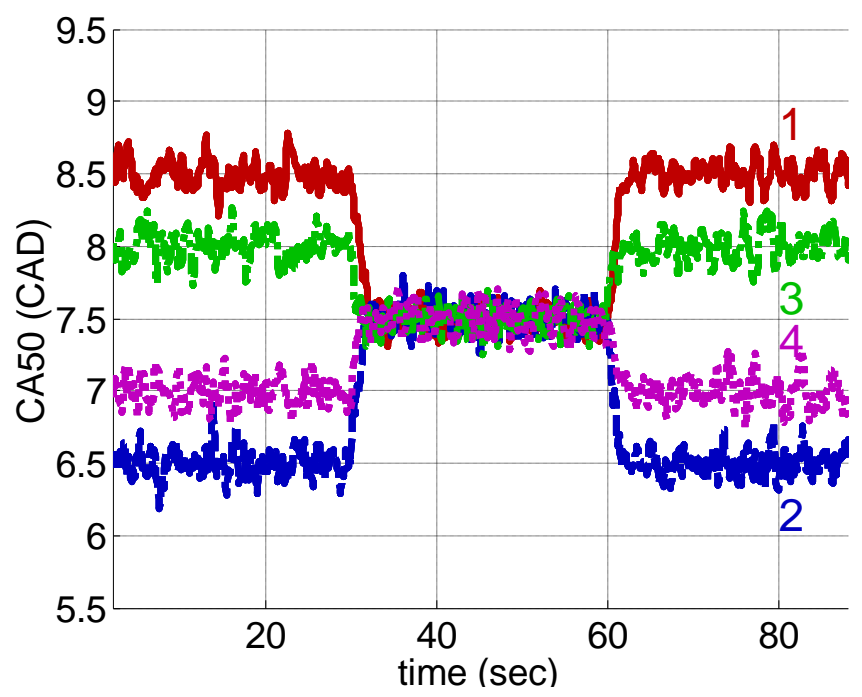

Figure 1.40. LQG-Integral Controller Experimental Results. Desired CA50:

[8.5,8,7,6.5] CAD 0-30, 60-90 sec; 7.5 CAD 30-60 sec. (Adapted from Souder, 2004) 


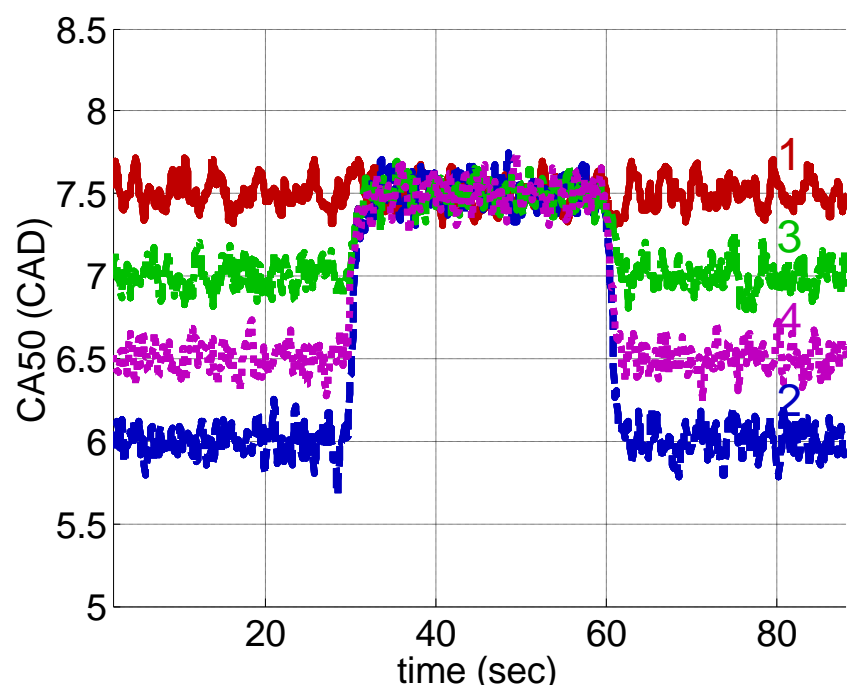

Figure 1.41. LQG-Integral Controller Experimental Results. Desired CA50:

[7.5,7,6.6,6] CAD 0-30, 60-90 sec; 7.5 CAD 30-60 sec. (Adapted from Souder, 2004)

The LQG-integral controller is guaranteed to be stable, from a result known as the separation theorem. However, even though the Kalman estimator has robustness properties similar to the LQ controller, the combination of an LQ optimal controller and Kalman filter has no robustness guarantees. To add robustness to the controller, $\mathrm{H}_{\infty}$ optimal control and $\mu$ synthesis are used. Even though the subspace-identified plant model could be used, a new plant model is derived using spectral analysis since it is more natural to obtain a frequency-domain uncertainty description from this model.

Performance weighting functions are chosen and used with the model to synthesize a mixed-sensitivity $\mathrm{H}_{\infty}$ optimal controller. While the $\mathrm{H}_{\infty}$ optimal controller significantly improves upon the robustness properties of the LQG-integral controller, it does not provide a method for directly incorporating model uncertainty into the control design. Given the high amount of uncertainty surrounding the HCCI combustion process, a method directly incorporating model uncertainty is desirable. To further improve robustness, spectral analysis and a multiplicative plant input uncertainty description are used to derive a "set" of models from experimental data. The model set includes a nominal plant and a group of perturbed plants that are "close" to the nominal plant. This model set is used to design $7^{\text {th }}$-order $\mu$-synthesis controllers that achieve robust performance.

Figure 1.42 shows the step response for one of the $\mu$-synthesis controllers. The response is slower than the previous controllers, though the tracking is still good. However, the controller is much more robust to model uncertainties and disturbances. Multi-cylinder HCCI engines are highly uncertain systems, so it is important to use a robust closed-loop controller. An $\mathrm{H}_{\infty}$ optimal controller, designed using a mixed-sensitivity approach, added a considerable amount of robustness to the basic LQG-integral controller, while maintaining equivalent performance. The frequency-domain system identification approach used here provides a direct estimate of the model uncertainty that can be directly incorporated into the controller design using $\mu$-synthesis to produce a robust controller that is guaranteed to meet or exceed its performance requirements. 


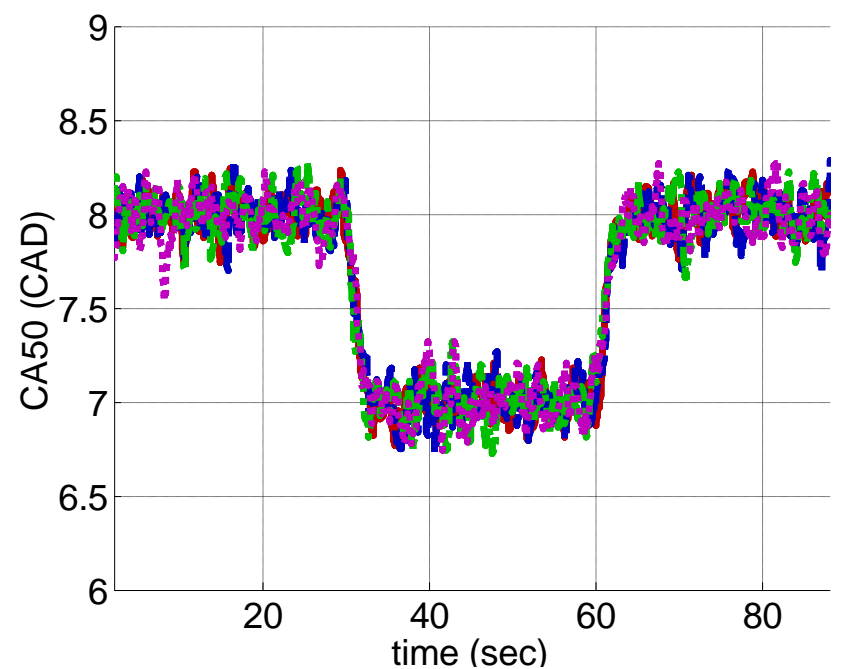

Figure 1.42. $\mu$-Synthesis Controller, Experimental Results, $\phi=0.36$. Desired CA50: 8 CAD 0-30, 60-90 sec; 7 CAD 30-60 sec. (Adapted from Souder, 2004) 


\section{VVA/DI-Based HCCI (Stanford)}

The focus of this work is to investigate the possibilities of using a fully flexible valve train or Variable Valve Actuation (VVA) to control the amount of residual gas retained in the cylinder and therefore the gas temperature and combustion timing. The Stanford single cylinder engine is camless as indicated in Figure 1.43 and is equipped with an electro-hydraulically actuated valve train which allows arbitrary valve timing and lift profiles.

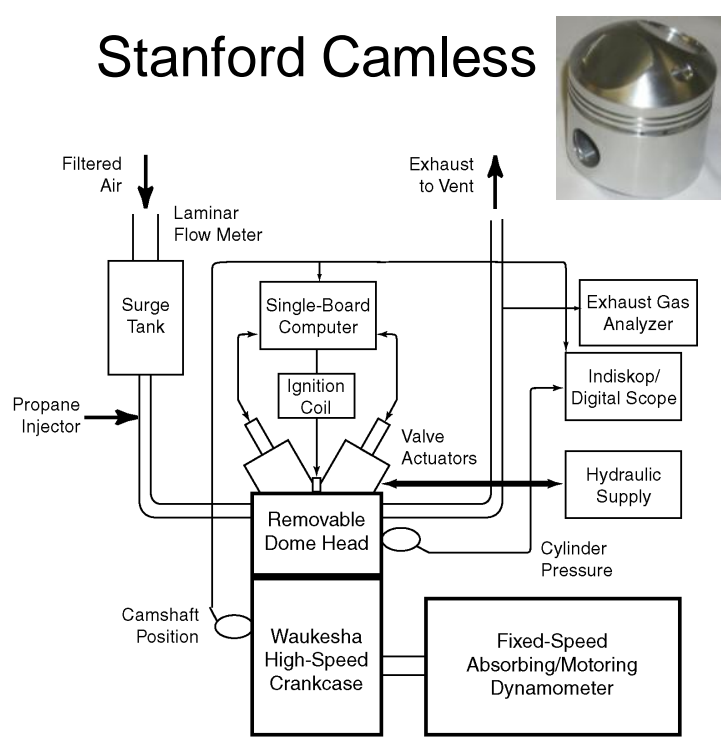

Figure 1.43. Schematic of the Stanford single cylinder CFR Removable dome head single cylinder engine equipped with an electro-hydraulically controlled Variable Valve Actuation (VVA) system.

\section{Effect of Inhomogeneities on HCCI}

Experiments with the VVA engine have assessed the effect of charge-residual inhomogeneity on HCCI performance. By altering valve profiles, the conditions that lead to mixing between fresh charge and residual are changed. In particular, using a low-lift, longduration intake valve profile instead of a full-lift, short-duration profile would tend to improve the mixing between charge and residual. The full-lift, short-duration profile forces all of the fresh charge induction to occur during a small fraction of the total intake stroke; a low-lift, longduration strategy spreads out the induction of fresh charge over the entire intake stroke. While results in the literature vary as to how charge-residual inhomogeneity changes combustion phasing, our experiments have shown that combustion phasing advances with flow conditions that would tend to enhance mixing between charge and residual. Figure 1.44 shows that little effect occurs until a lift-duration combination of approximately $0.31 \mathrm{~cm} / 180 \mathrm{CAD}$, at which point phasing advances and peak pressure rises. These changes are consistent with an improvement in charge-residual homogeneity. 
The low-lift, long-duration intake profiles also allow a wider load range with no other changes in engine configuration, and allow operation at lower loads/higher dilution. Figure 1.45 shows calculated residual fraction for two different intake valve profiles: a low-lift, long-duration profile with variable lift and a full-lift, short-duration intake profile with variable duration. Note that the low-lift profile, which would tend to improve mixedness between charge and residual, shows a greater range of dilution tolerance, and therefore, a wider operating load range that extends to lower loads (higher dilution).

\section{Independent Phasing and Load Control}

In order to independently control both phasing and load, at least two degrees of freedom are required. Using VVA technology to affect valve profiles, we have investigated two successful choices for these degrees of freedom. By using a fixed and delayed exhaust valve profile, the intake valve opening (IVO) time and the intake valve closing time (IVC) may be used to control load and phasing independently. With delayed IVC, the charge-residual mixture is intentionally forced back in the intake port, which has the additional affect of lowering the effective compression ratio relative to the expansion ratio The IVO time determines the amount of induced charge, and thus sets the composition by controlling the ratio of charge to residual (residual fraction). By using IVO and IVC to control the residual fraction and effective compression ratio, respectively, load variation was demonstrated independently of combustion phasing, which was held fixed (Figs. 1.46-1.47).

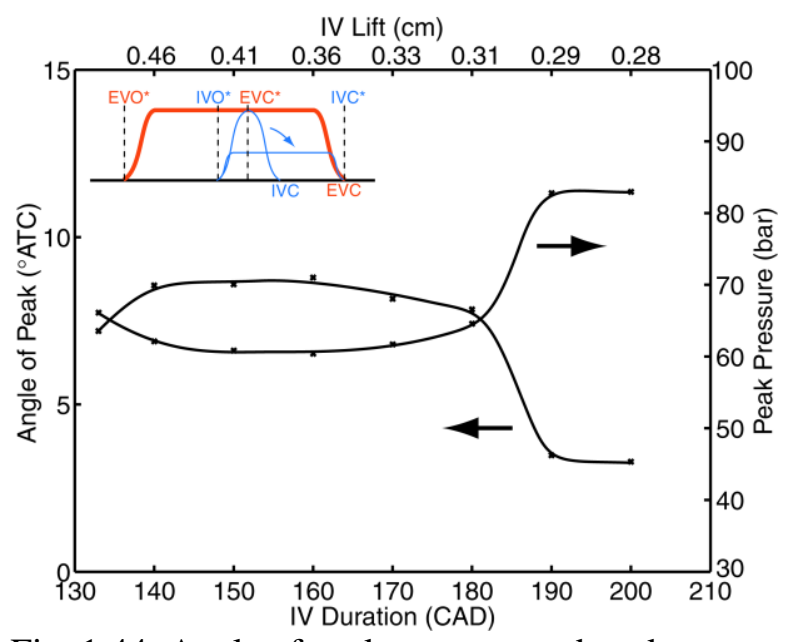

Fig. 1.44: Angle of peak pressure and peak pressure for an intake valve profile that transforms from a full-lift, short-duration profile into a lowlift, long-duration profile.

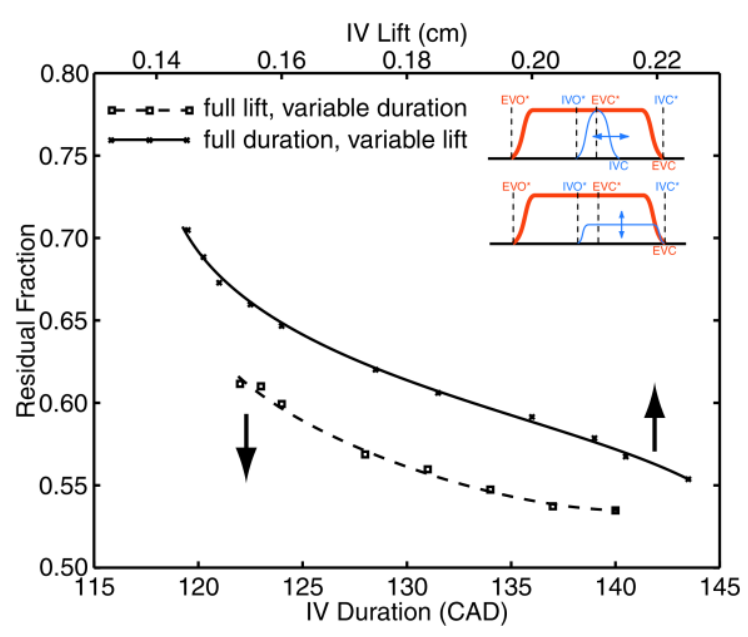

Fig.1.45. Calculated residual fraction for two different intake valve profiles. Low lift profile shows greater range of dilution tolerance. 


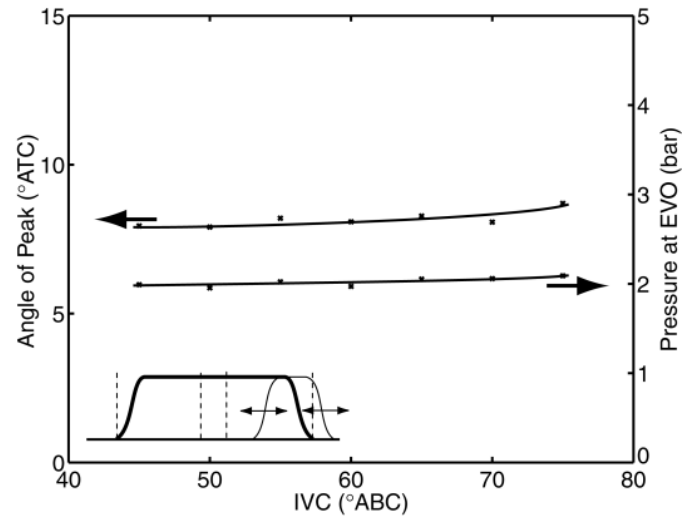

Fig. 1.46: Angle of peak pressure and pressure at exhaust valve opening (EVO) with variable IVO (controlling residual fraction) and IVC (controlling effective compression ratio). As IVC was delayed, the IVO was adjusted to hold the angle of peak pressure fixed at approximately $8^{\circ}$ ATC.

Another choice for control parameters is delayed IVC and delayed EVC. Using a model-based LQR control scheme, control of peak pressure has been previously demonstrated (Shaver et al., 2005a, 2005b). Peak pressure scales closely with load, therefore, current control efforts have focused on peak pressure as a reasonable surrogate for load. Control of peak pressure and phasing has been recently demonstrated by using a decoupled scheme, in which peak pressure is controlled on a cycleto-cycle basis while phasing is controlled on an intentionally slower time scale. Figure 1.48 shows the experimental results. It is interesting to note that, as expected, at later phasing the peak pressure has more cycle-tocycle variation as the process get harder to control. In order to control phasing more quickly, the peak pressure portion of the controller would need to take into account information about cycle-to-cycle changes in combustion phasing.

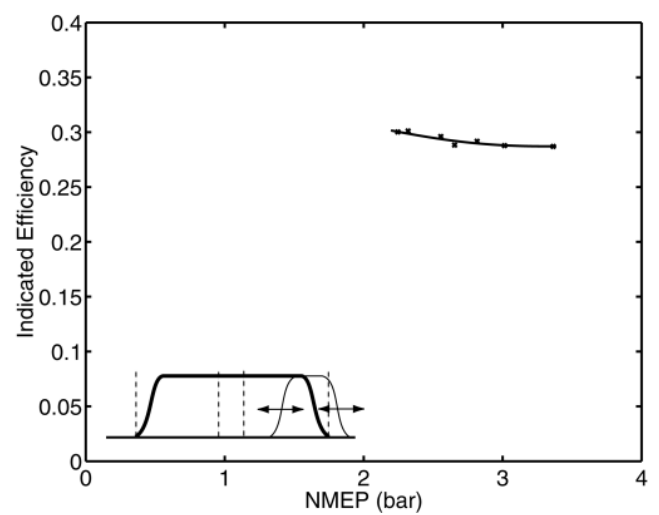

Fig. 1.47: Indicated efficiency as a function of load for the operating points shown in Fig. 1.46. Note that, while phasing is being held fixed, the engine operates across a significant load range (2.2 bar to 3.4 bar) while maintaining relatively constant efficiency.

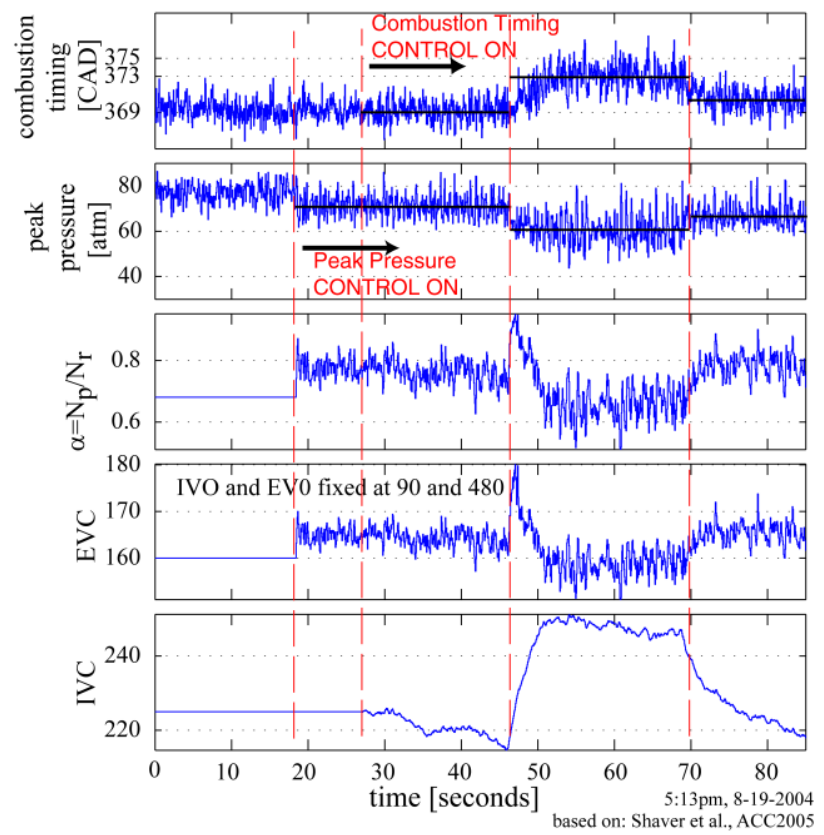

Fig. 1.48: Combustion timing and peak pressure shown as a function of time using delayed IVC and EVC valve timings. The changes in IVC and EVC are also shown along with $\alpha$, an internally calculated control parameter representing the molecular ratio of residual to fresh charge. The controller is able to achieve independent tracking of peak pressure and combustion timing. 


\section{Task 2: Full Cycle and System Modeling Tools (UM)}

\section{System Modeling Tools}

\section{RPM scaling effects on ignition timing}

Because of the strong coupling between temperature and chemistry, there is an inherent difficulty in trying to distinguish between the thermodynamic and the chemical effects in an HCCI engine. Interpretation of experiments and models is complicated by this coupling. Recently we have attempted to isolate the chemical kinetics effects in the framework of a zerodimensional, thermo-kinetic model. The goal of this study was to explore and identify the factors determining combustion phasing in HCCI engines; in particular it is hoped that by recasting the conditions to those encountered at TDC, simplifications and scaling effects will be found.

In order to focus on chemistry and timing around TDC, we used the Chemkin subroutines with fixed composition and under adiabatic conditions in a time varying volume controlled by the slider crank equation. The cases considered are based on two engines: the Sandia engine (Sjoberg and Dec, 2003), which achieves ignition by high compression ratio and inlet heating, and the UM engine (Chang et al., 2004) which utilizes a rebreathing exhaust valve cam to reinduct large quantities of sensible heat from the exhaust gases. The composition and thermodynamic conditions for these two cases were determined with a single zone full cycle simulation tool (HCCIES) developed at UM. This tool takes into account the real world effects of breathing and heat transfer and was matched to the experimental engine operating points with conditions in Table 2.1. Once this was done all further calculations were carried out in a separate single zone code for the closed cycle. As indicated in Table 2.2, composition and pressure at TDC were fixed for each engine, while the initial temperature was varied. The temperature at TDC used for reference was calculated by a parallel adiabatic computation with fixed composition and no reaction. The kinetic calculations used the detailed mechanism for isooctane developed by Curran et al., (2002).

Table 2.1. Model parameters for base conditions

\begin{tabular}{|l|l|l|}
\hline Engine & Sandia & UM \\
\hline Combustion Initiation & CR, Tinlet & Rebreathing \\
\hline Bore (mm) & 102.0 & 86.0 \\
\hline Stroke (mm) & 120.0 & 94.6 \\
\hline CR & 16.6 & 13.0 \\
\hline L/R & 3.2 & 3.22 \\
\hline RPM & 1200 & 2000 \\
\hline Fuel & isooctane & isooctane \\
\hline Pinlet, Pexhaust & 1 bar & 1 bar \\
\hline $\begin{array}{l}\text { Tinlet for TDC comb. } \\
(\mathrm{K})\end{array}$ & 422 & 390 \\
\hline
\end{tabular}


Table 2.2 Input conditions for Adiabatic Model runs

\begin{tabular}{|l|l|l|}
\hline Engine Case & Sandia (S40) & UM (UM23) \\
\hline Phi & 0.26 & 0.784 \\
\hline EGR (mass \%) & 4.9 & 36.3 \\
\hline IC8H18 (mole frac.) & 0.00413 & 0.00815 \\
\hline O2 & 0.20627 & 0.14587 \\
\hline N2 & 0.78598 & 0.76706 \\
\hline CO2 & 0.00170 & 0.03714 \\
\hline H2O & 0.00192 & 0.04178 \\
\hline P_270 (bar) & 1.80 & 1.52 \\
\hline RPM & 1200 & 2000 \\
\hline P_TDC (bar) & 40 & 23 \\
\hline
\end{tabular}

The base temperature sweep results are shown in Fig. 2.1 for the Sandia engine. Similar plots were obtained for the UM engine.
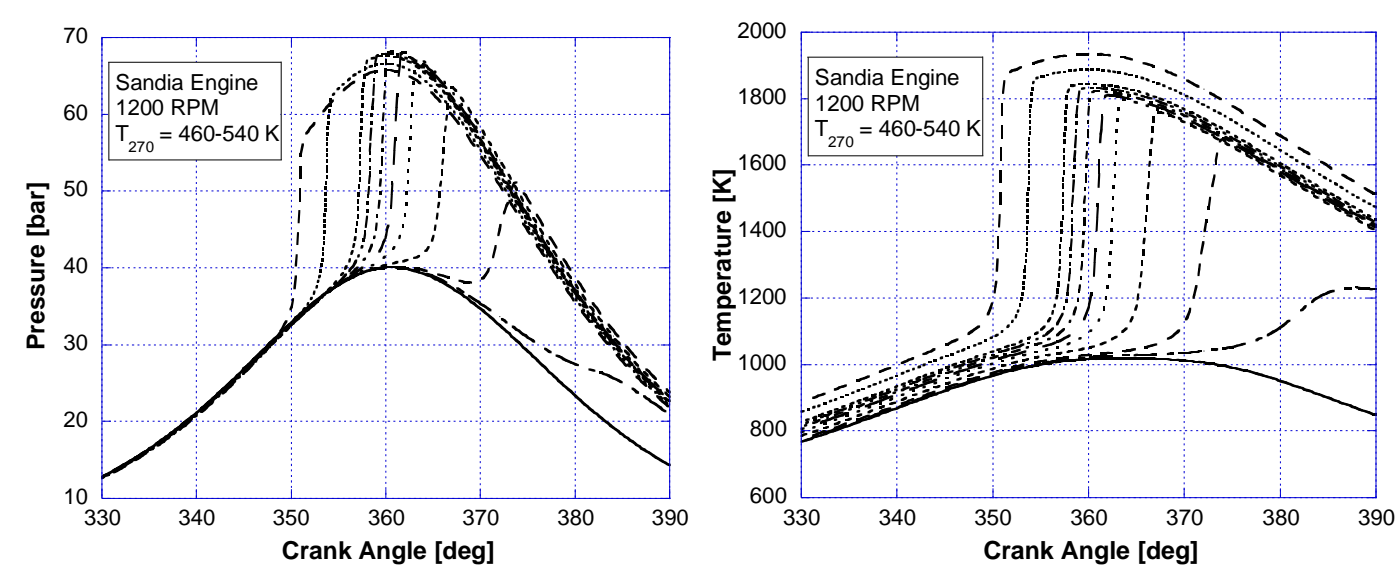

Fig. 2.1. Pressure and Temperature traces from a single zone model over a temperature sweep for Sandia engine at 1200 RPM.

Calculated combustion phasing results for both engines are shown in Fig. 2.2. Plotted is computed motoring temperature at $\mathrm{TDC}, \mathrm{T}_{\mathrm{TDC}}$, vs. the $10 \%$ mass fraction burned crank angle denoted by $\theta_{10}$. Sweeps for four speeds are shown from 1200 to $8000 \mathrm{rpm}$. 


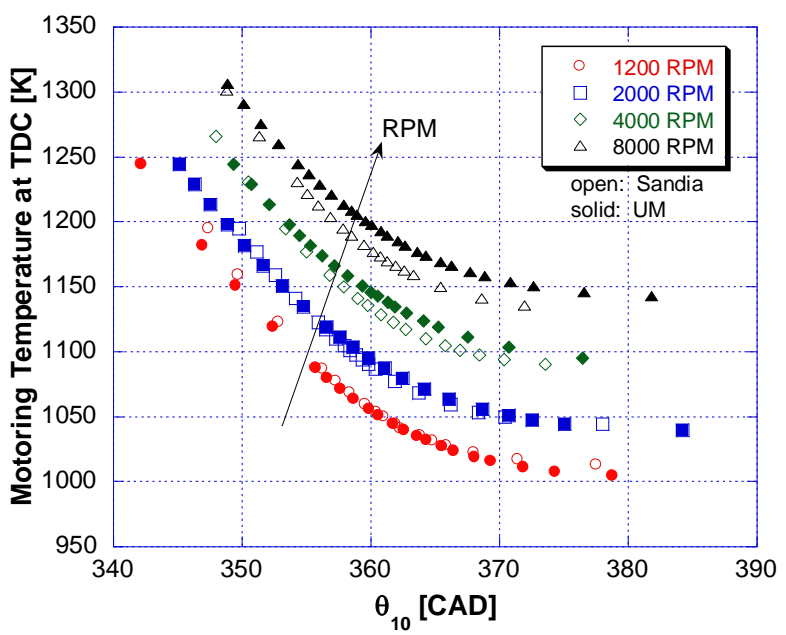

Fig. 2.2. Motoring temperature at TDC as a function of $\theta_{10}$ for Sandia and UM engine at different RPM.

The curves show the expected decrease in temperature with later combustion phasing, and flatten out after TDC as combustion becomes slow and eventually incomplete. For the higher RPM's, higher temperature is required in order to complete the ignition process in the diminished time available. The two engines show similar trends. However the UM engine requires a greater boost in TDC temperature than the Sandia engine as speed is raised.

The results of Fig. 2. 2 are replotted in Fig. 2.3; however instead of the motoring temperature at TDC, the corresponding constant volume ignition delay time is plotted in crank angle degrees. (This ignition delay time was evaluated using the same computer program and kinetics but with a fixed volume and corresponds to typical kinetics benchmark data obtained in a shock tube or rapid compression machine).

The curves at different RPM collapse into one curve for each engine and show that the equivalent ignition delay time at TDC conditions necessary for TDC ignition is about 9 degrees for the Sandia engine and 11 degrees for the UM engine.

In supporting work not shown here

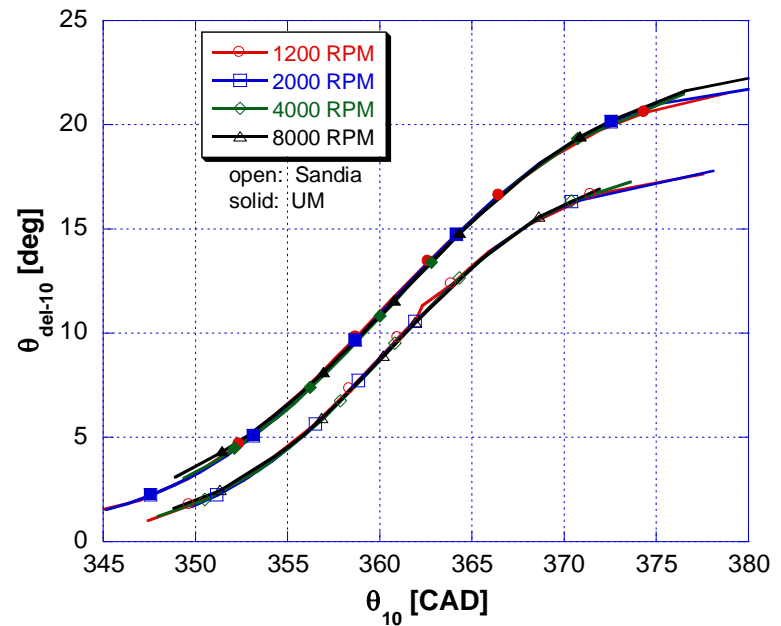

Fig. 2.3. Ignition delay (tau10) expressed in $\mathrm{CAD}$ as a function of $\theta_{10}$ for the Sandia and UM engines at different RPM. we have found that the exact position of the curves depends on compression ratio, polytropic exponent (heat transfer) and to a lesser extent the effective activation energy of the ignition delay kinetics. Thus the UM engine requires a longer ignition delay due to the longer residence time near TDC conditions afforded by the lower 
compression ration (13 vs.16.6). The required ignition delay, expressed in crank angle terms, appears to scale with RPM for a given combustion phasing.

Combustion efficiency however does not scale with RPM as shown in Fig. 2.4. Once partial burning sets in, higher RPM's give lower combustion efficiency for a given ignition timing.

Finally we made a preliminary validation of these predictions against available data for the two engines using premixed isooctane fuel. Limited experimental data sets at 1200 RPM for the Sandia engine and 2000 RPM for the UM engine were carefully analyzed with GT-power to obtain an estimate of average motoring temperature at TDC. A maximum gas temperature was estimated to be $6 \%$ higher than the average cylinder temperature based on CFD simulation results for the Sandia engine (Sjoberg et al., 2004). Fig. 2.5 shows the predicted $\theta_{10}$ curves along with the corresponding experimentally derived points.

The results are plausible and show good agreement over a limited range and within the accuracy of the estimated TDC temperatures. Recently we replicated these results using a knock integral approach (Babajimopoulos et al., 2007). This validates the widespread use of knock-integral methods for predicting ignition timing in fast HCCI engine and system models.

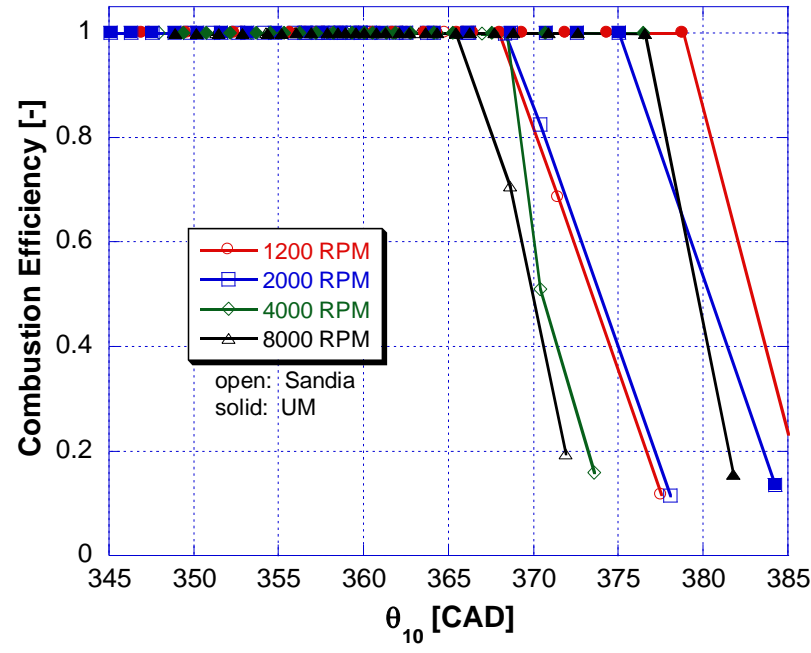

Fig. 2.4. Combustion efficiency as a function of $\theta_{10}$ for Sandia and UM engine at different RPM.

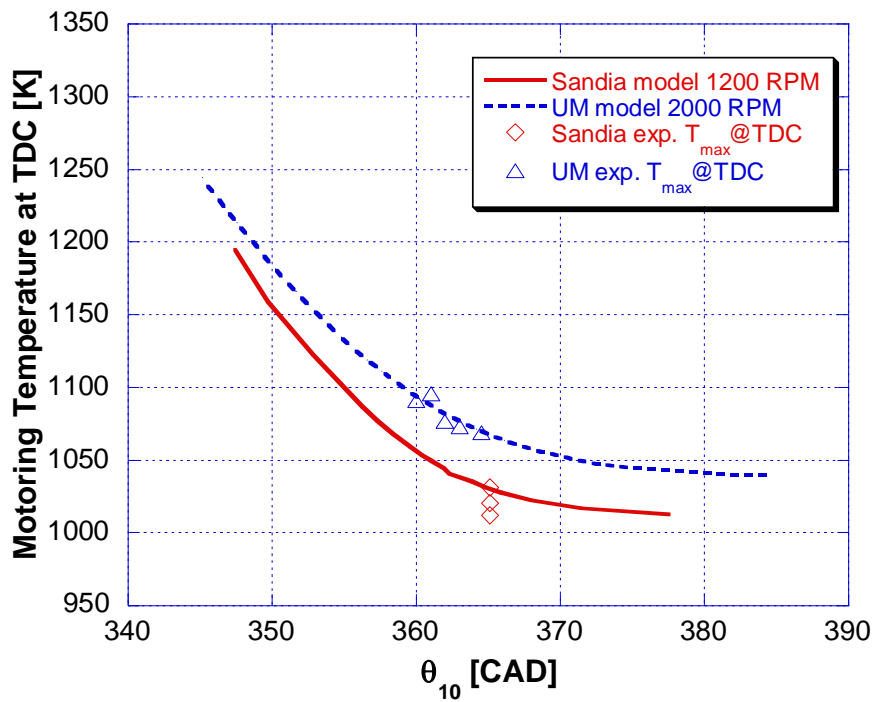

Fig. 2.5. Motoring temperature at TDC as a function of $\theta_{10}$ for Sandia engine (1200 RPM) and UM engine (2000 RPM). Lines: kinetics results; Points: Estimated experimental maximum temperature in cylinder. 


\section{Knock-Integral in GT Power Engine Model}

As the basis of our GT Power engine system model we have employed a knock integral method (see above) to predict ignition timing. In early testing we were able to demonstrate good agreement with the isooctane engine data of Sjoberg and Dec (2003) for trends in inlet temperature and equivalence ratio. The ignition delay expression was taken directly from the work of the consortium kinetics team reported in Section 4, and is based on experimental shock tube and RCM data for isooctane:

$$
\tau=2.766 \times 10^{-3} \cdot P^{-0.99} \cdot \phi^{-0.87} \cdot \chi_{O_{2}}^{-1.57} \cdot \exp \left(28200 / R_{[\text {call mol K K] }} T\right)
$$

where $\tau$ is the ignition delay time in [ms], $\mathrm{P}$ is pressure [atm], $\mathrm{T}$ is temperature $[\mathrm{K}], \phi$ is the equivalence ratio, and $\chi_{O 2}$ is the oxygen mole fraction in [\%].

The GT model was tested against experimental transient HCCI data obtained from the MIT colleagues working with a VVT system (see Task 1). The variable valve timing was relatively simple to simulate within GT Power. The start of combustion was calculated by integrating $1 / \tau$ beginning at intake valve closing. When this integral reached 1, ignition was assumed to have occurred and combustion took place based on the prescribed Wiebe function. The burn duration defining the Wiebe function was taken from the experimental data and shown in Fig 2.6. Combustion efficiency was approximately constant at $95 \%$ over the range studied. This simple combustion model has been refined considerably since this study (see below) but the general features of

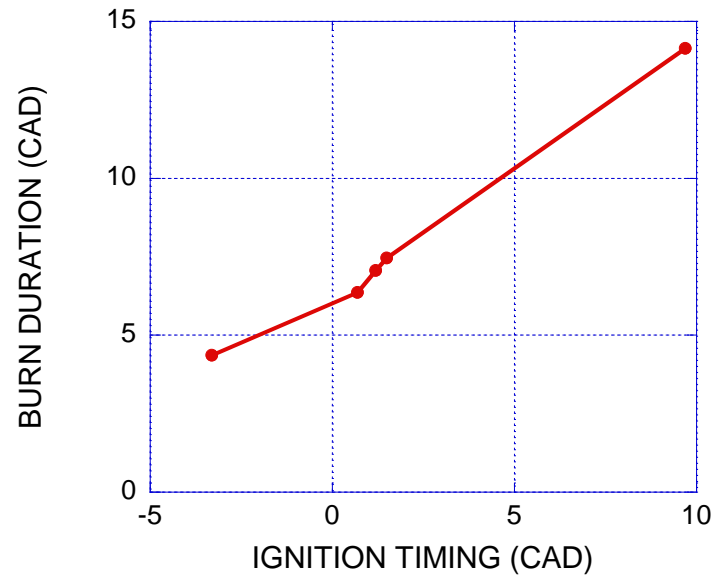

Fig. 2.6 preliminary burn duration correlation used for GT Power simulation. Data from UM test engine. its behavior are similar to those displayed here.

The case modeled was a transition from SI to HCCI operation. In spark ignited mode the engine was operated with zero overlap near TDC but with late intake valve closing. To initiate the transition, negative overlap was introduced to increase EGR while the IVC was advanced to maintain approximately constant fresh charge intake and constant load. The spark was kept on for one cycle after transition. The valve timing used is shown in figure 2.7. In the simulation the combustion timing was fixed up to and including cycle 15 . For cycle 16 and above, the knock integral was used along with the correlation to determine the combustion phasing. 


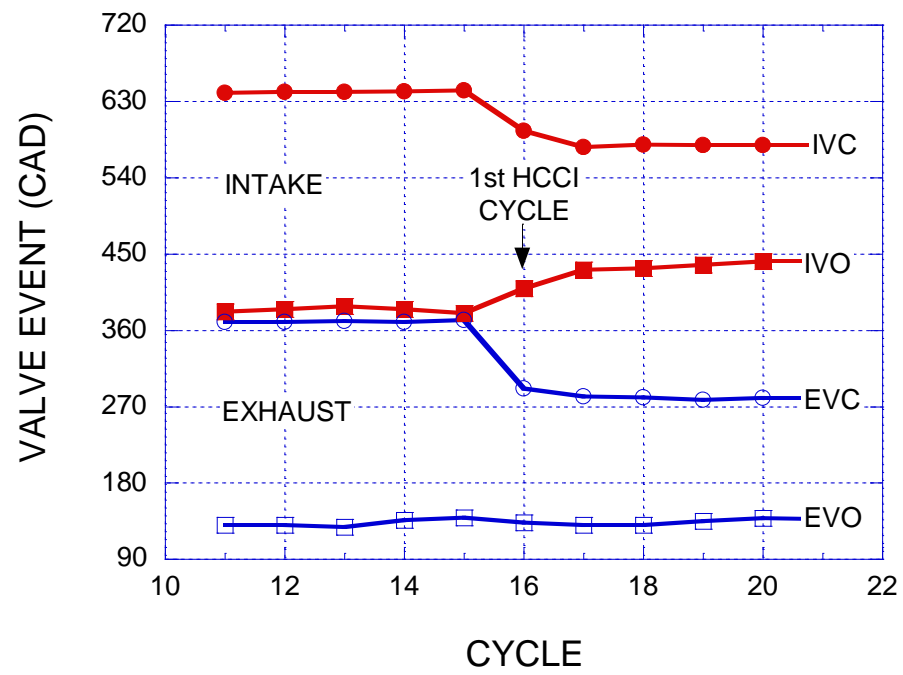

Figure 2.7. Actual valve event timing for SI-HCCI transition in MIT engine; and used as input to the GT model

The simulation results are shown in Figure 2.8 where the sequence of pressure curves is displayed, the experimental set on the left and the modeled curves on the right. Note that the cycle number sequences are offset in the figures; however the first curve shown in each case is the last SI cycle before transition begins.

EXPERIMENT (MIT)

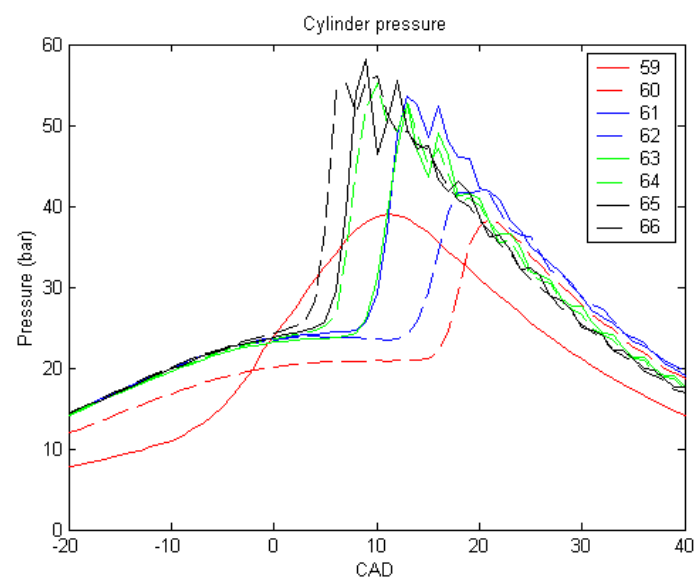

MODEL (UM)

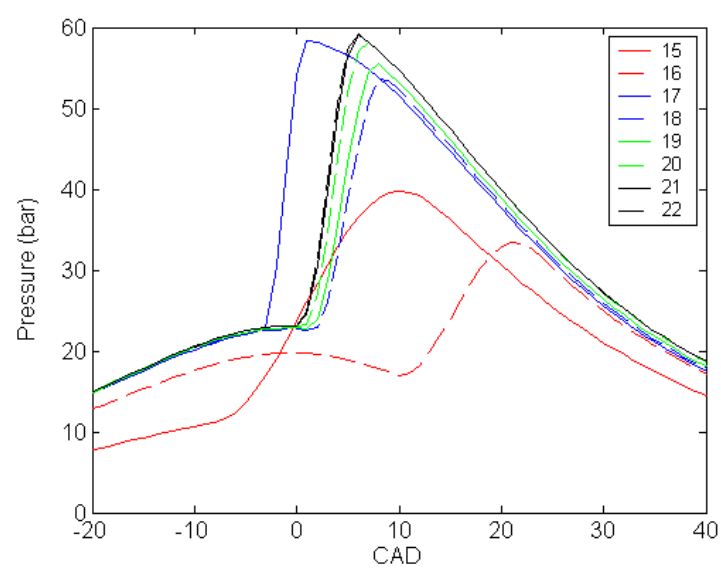

Figure 2.8. Pressure curves for SI-HCCI transition in the MIT engine. Experimental curves on left, GT knock integral model on the right. In each case, the first cycle displayed is the last before the transition.

The model is remarkably similar to the experiment, duplicating the late combustion of the first HCCI cycle, and the eventual stabilization of combustion phasing near TDC. This effect is due to the emptying and filing time scale affecting the residual fraction and is on the order of 310 cycles. 
In Task 1, the MIT team observed sensitivity of the SI-HCCI transition to valve timing change strategies. To explore this with our model we set up three transitions, requiring one, two and three cycles (1 STEP, 2 STEP and 3 STEPs) to fully shift from SI to HCCI phasing. The valve strategies and resulting calculated IMEP's for each cycle are shown in Figure 2.9
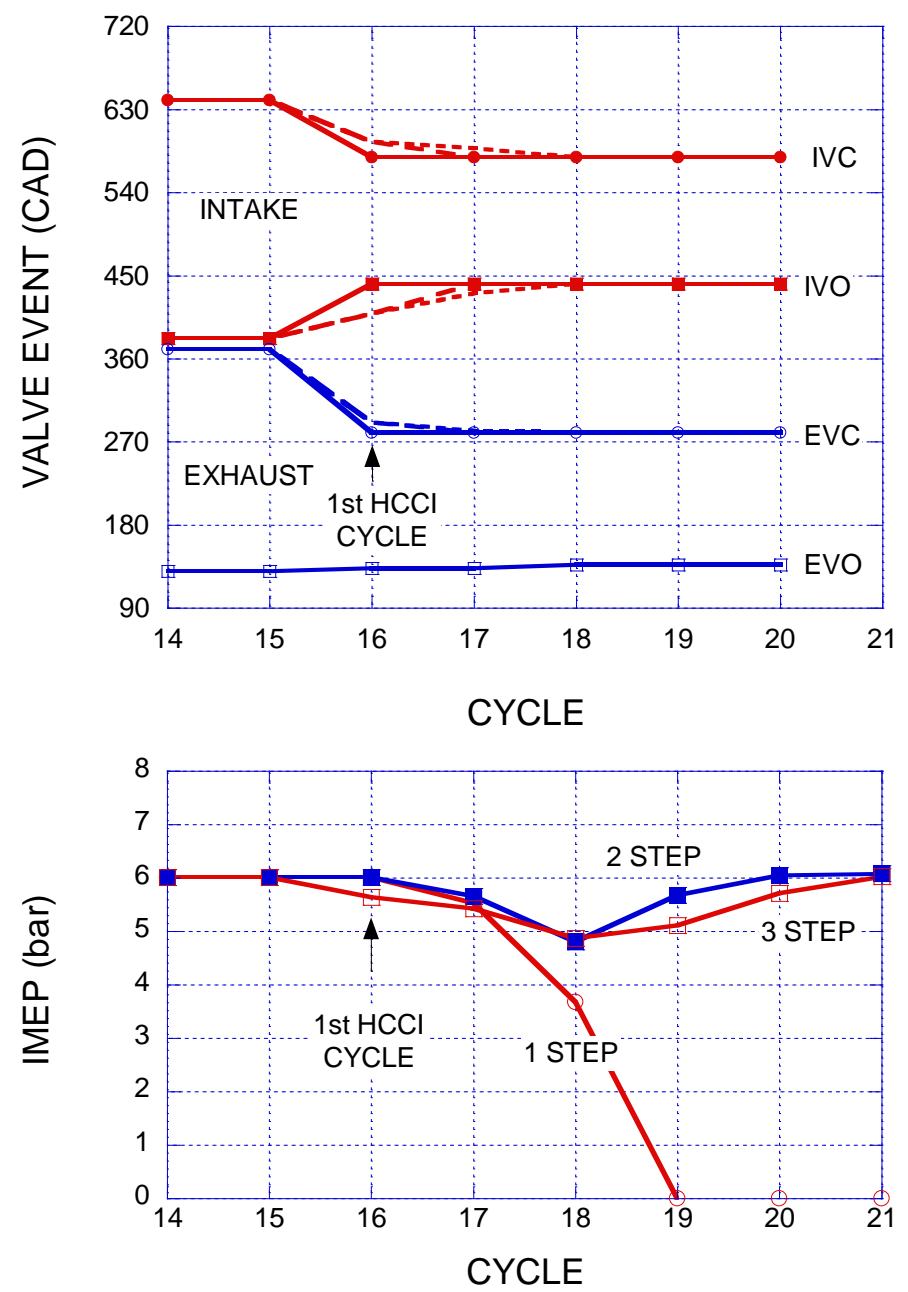

Fig. 2.9 Three valve change strategies (upper figure) used in simulation of SI-HCCI transition with GTpower model, and resulting IMEP's (lower figure) for each cycle.

The results are qualitatively similar to the observations noted in Section 1.1. It is interesting that the most abrupt strategy (1 STEP) results in the earliest effect at cycle 16, however it is unstable and eventually misfires. The more gradual valve change strategies are both stable. The reason for this behavior appears to depend on the rebreathing dynamics since wall temperatures were held constant for all cycles. 


\section{Improved burn rate correlations}

In order to develop a more realistic correlation for burn rates for use in the system level simulations we took a set of 28 points shown in figure 2.10 obtained from the UM HCCI engine with premixed gasoline over a range of parameters varied around a base point. These variables include speed, load, inlet temperature, coolant temperature, and $\mathrm{A} / \mathrm{F}$ at constant load. Although the range was not large for these variables, it was sufficient to cover a range of combustion phasing from overly advanced to late enough to cause COV of IMEP to rise to $3 \%$.
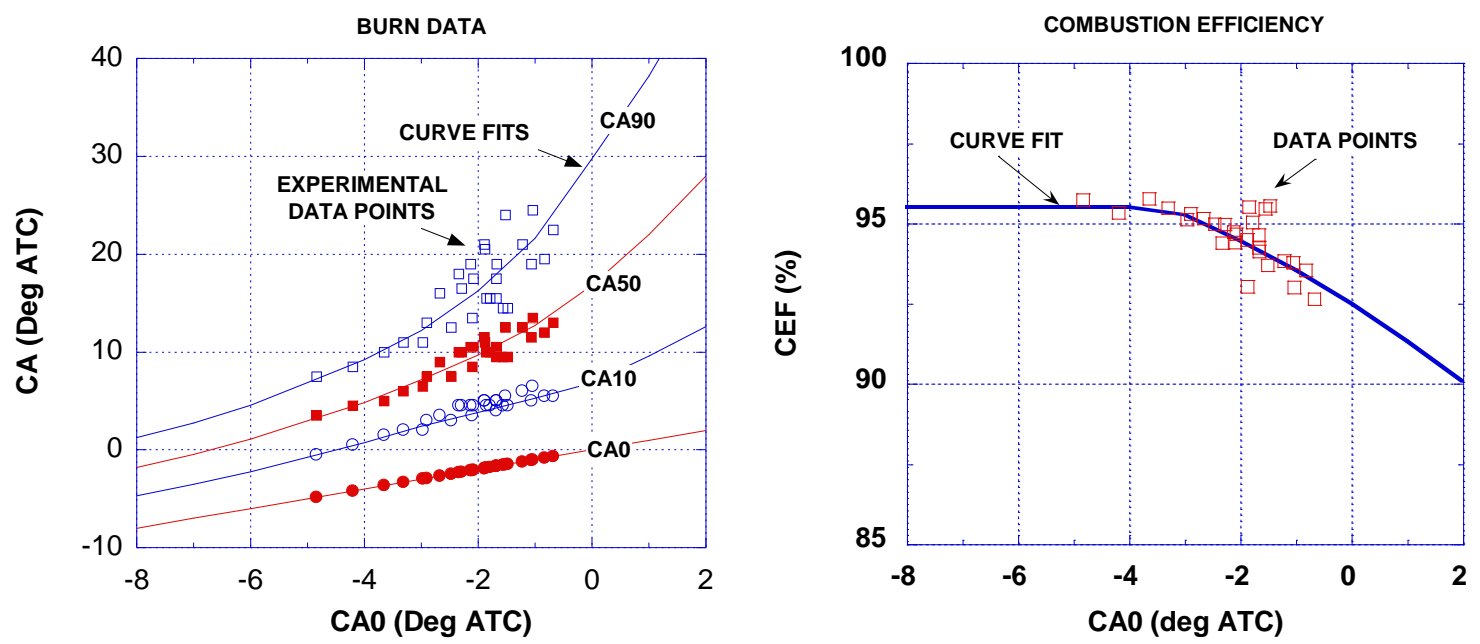

Fig. 2.10. Experimental burn data (LEFT) and combustion efficiency (RIGHT) for the UM engine.

Average pressure traces at each point were analyzed by a mass fraction burned program to reveal start of combustion, $10 \%, 50 \%$ and $90 \%$ burned (denoted by CA0, CA10, CA50, and CA90). These percentages are relative to the total energy released which is less than $100 \%$. Combustion efficiencies were determined by exhaust gas analysis.

Fig. 2.10 shows the experimental values of $0,10,50$, and 90 percent burned for all the points plotted against the 0 percent burned crank angle and the combustion efficiency data. Also shown are curve fits to the data. Although there is some scatter in the data it is clear that the most important variable determining the burn parameters is the combustion phasing.

The curve fit lines in Fig. 2.10 were derived from a Wiebe function with parameters, $\Delta \theta$ and $\mathrm{w}$ chosen to best represent the data. The Wiebe function used here for normalized mass fraction burned $\mathrm{x}$ is:

$$
x_{N O R M}=1-\exp \left[-\left(\left(\theta-\theta_{0}\right) / \Delta \theta\right)^{w+1}\right]
$$


where $x_{\text {NORM }}$ is multiplied by the combustion efficiency to obtain absolute values. $\Delta \theta$ has dimensions of crank angle degrees and generally characterizes the duration of burn while the exponent $\mathrm{w}$ is a shape factor which affects primarily the tail of the burn curve.

The values used for $\Delta \theta$ and $\mathrm{w}$ are given in Fig. 2.11. It is interesting that the shape factor changes significantly over the central range of Fig. 2.10 where actual data is available. Beyond that we have set $\mathrm{w}$ constant for lack of any better data. The decrease in $\mathrm{w}$ for the later burns indicates a greater amount of time is spent in the 50$90 \%$ part of the burn curve. This may be due to increased cooled gas in the boundary

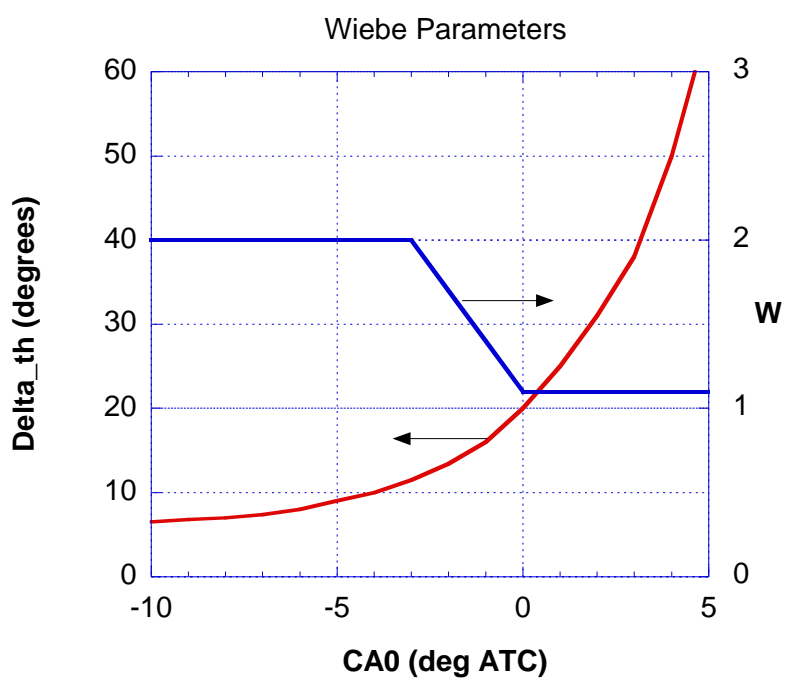

Fig. 2.11. Wiebe parameters chosen to give the best fit to the combustion data in Fig. 2.10. layers later in the cycle.

The curve fit for combustion efficiency CEF in Fig. 2.11 is given by:

$$
\begin{aligned}
\operatorname{CEF}(\%) & =95.5 ; & \text { CA0 }<-3.33 \\
& =92.5-1.1(\mathrm{CA} 0)-0.06(\mathrm{CA} 0)^{2} ; & \mathrm{CA} 0 \geq-3.33
\end{aligned}
$$

Together, these parameterizations allow the determination of heat release rate as a function of start of combustion. The expressions have been incorporated into our engine-invehicle system model which was used for the transient wall temperature simulations described in Task 1 above.

\section{Full Cycle Thermo-Kinetic Multi-Zone Simulation}

A new simulation tool for HCCI combustion analysis was developed in collaboration with LLNL (Babajimopoulos et al., 2005). A CFD code (KIVA-3V) was fully integrated with a multi-zone model that utilizes detailed chemical kinetics. At each computational timestep, the multi-zone model provides KIVA-3V with the necessary energy source terms for each cell, by solving the detailed chemical kinetics for groups of cells (zones) with similar properties. The methodology is an extension of previous work in that it can now account for both temperature and composition gradients in the cylinder throughout the whole cycle. To achieve that, a method for tracking the equivalence ratio in each cell during combustion and a technique for mapping the solution back and forth between KIVA-3V and the multi-zone model were developed. The advantage of the new model is that it can perform open or closed cycle CFD simulations with detailed chemistry at a reasonable computational time (10-12 hours), while maintaining good accuracy. 
The new model can be used to investigate the effect of realistic temperature and concentration gradients within the cylinder. Figure 2.12 shows temperature profiles for a typical case at $2000 \mathrm{rpm}$. The figure shows the cumulative temperature distributions for several crank angles during the combustion event $(0,10,20,30$ Deg ATC) along with the maximum temperature envelope. As expected, because of the temperature gradients, the total burn durations are much longer than a corresponding single zone model. More importantly, again because of the thermal boundary layer, a significant part of the charge never attains a local temperature sufficiently high $(1100 \mathrm{~K})$ to ignite. Consequently, the combustion efficiency for this case is only about $80 \%$.

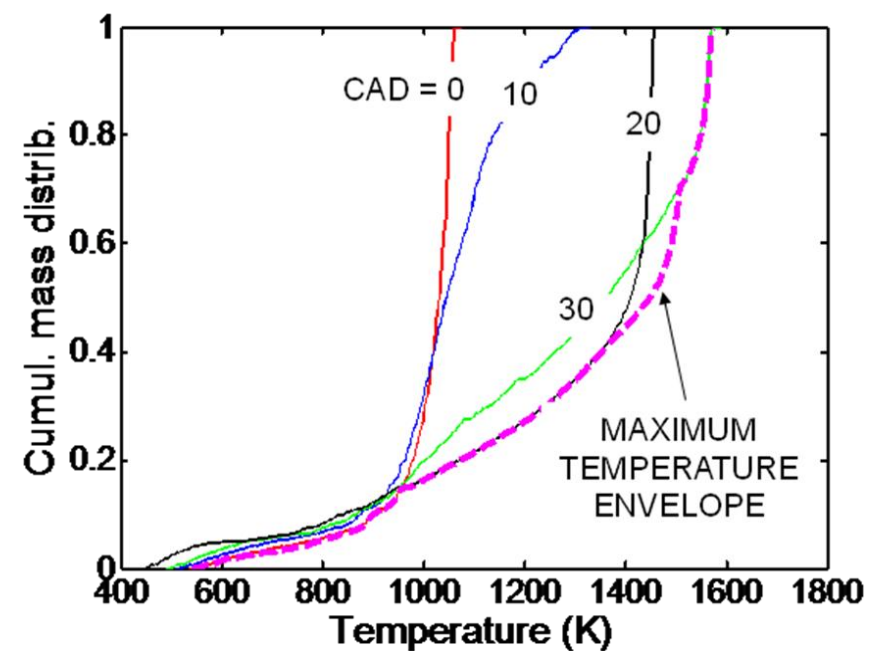

Fig. 2.12. Cumulative temperature distribution at several crank angles during the combustion event.

A practical goal of this study is to utilize the Multi-Zone model to develop realistic correlations for burn duration and combustion efficiency which can be used in systems models and control analysis. These correlations would take into account engine operating variables as parameters, but more importantly could identify parametric effects of design changes such as piston shape and compression ratio.

Initial results have been obtained for an intake temperature sweep. ( $\left.\mathrm{T}_{\mathrm{IVC}}\right)$. The KIVA$3 \mathrm{~V}$ calculations were performed on $2 \mathrm{D}$ grids to provide adequate wall layer resolution without compromising computational time. The head and the piston are flat and the topland crevice is included. Also to avoid long runtimes, the calculations only covered the closed cycle portion of the event. The runs start at IVC $\left(150^{\circ} \mathrm{BTDC}\right)$, at which time the composition and temperature in the cylinder are uniform, and stop 50-100 ${ }^{\circ}$ ATDC. The model parameters are given in Table 2.3. 


\section{Table 2.3. Model parameters for KIVA-3V simulations}

\begin{tabular}{|l|l|}
\hline Bore $(\mathrm{mm})$ & 90.0 \\
\hline Stroke $(\mathrm{mm})$ & 105.0 \\
\hline CR & 16.0 \\
\hline L/R & 3.2 \\
\hline Crevice width $(\mathrm{mm})$ & 0.5 \\
\hline $\mathrm{V}_{\text {crevice }} \mathrm{V}_{\text {TDC }}$ & $\sim 4 \%$ \\
\hline RPM & 2000 \\
\hline Fuel & Isooctane \\
\hline $\mathrm{P}_{\text {IVC }}$ & 1.1 bar \\
\hline $\mathrm{T}_{\text {IVC }}$ & Varied \\
\hline Equivalence Ratio & 0.26 \\
\hline EGR & $5 \%$ \\
\hline
\end{tabular}

The temperature sweep results are presented in Figures 2.13 and 2.14. Figure 2.13 shows how the ignition timing, expressed as location of $1 \%$ mass fraction burned $\left(\mathrm{CA}_{01}\right)$, is advanced with increased $\mathrm{T}_{\mathrm{IVC}}$. In the same figure, it can be seen how combustion efficiency deteriorates as ignition timing is retarded. If ignition starts after $2^{\circ}$ BTDC, the combustion efficiency drops rapidly.

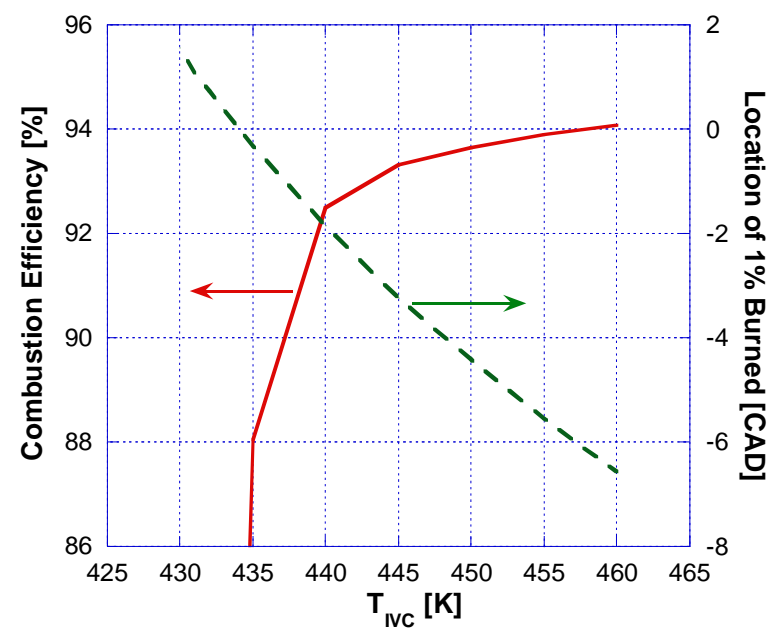

Fig. 2.13. Combustion efficiency and location of $1 \%$ mass fraction burned as a function of temperature at intake valve closing. 


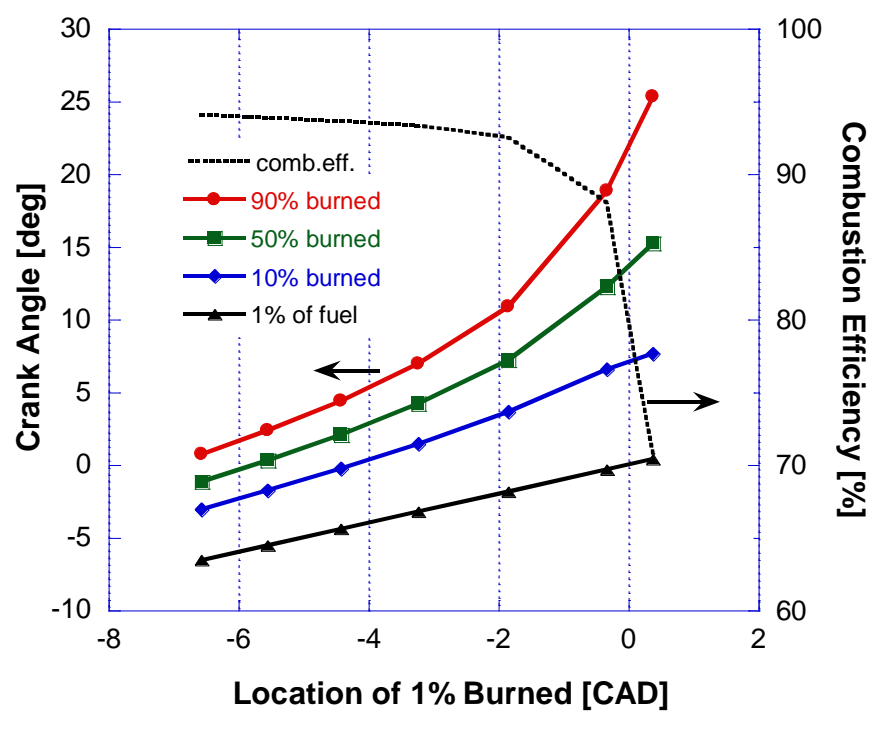

Fig. 2.14. Location of $10 \%, 50 \%$ and $90 \%$ mass fraction burned and combustion efficiency as a function of the location of $1 \%$ mass fraction burned.

Figure 2.14 shows the location of $10 \%, 50 \%$ and $90 \%$ mass fraction burned $\left(\mathrm{CA}_{10}, \mathrm{CA}_{50}\right.$, $\mathrm{CA}_{90}$ respectively) and combustion efficiency as a function of $\mathrm{CA}_{01}$, the crank angle when $1 \%$ of the total fuel is burned and where we define ignition. The other crank angles refer to the percentages burned relative to the total chemical energy actually released. Figure 2.14 also shows that the burn duration gets longer as ignition is retarded.

These results represent quite well the observed experimental trends (see Fig. 2.10) and demonstrate the effect of ignition timing on burn rate and combustion efficiency under a specific set of operating conditions. As more parametric studies are performed, the effect of other factors such as load, speed, etc., will also be explored as well as design parameters. The goal is to produce a large pool of "numerical experiment results", which will be used to provide understanding of the complex interactions among variables and for the development of better correlations. 


\section{Task 3: Investigation of Chemical Kinetics to Model the HCCI Combustion Process (UM, Stanford, Berkeley, MIT)}

\section{Development of reduced kinetics for HCCI Engine Combustion (MIT)}

\section{Reduced Chemical Kinetic Models}

The goal of this task was to develop chemical kinetic models for HCCI along with methods that would allow one to use the existing large $(\sim 5000$ chemical reactions $)$ chemical kinetic models for fuel combustion in HCCI engine simulations. We addressed this challenging task from three angles:

1) Improve the chemistry;

2) Automate the model reduction step; and

3) Improve the numerical solvers.

To improve the chemistry, we started from the existing fuel chemistry models (e.g. the LLNL PRF model of Curran et al., 2002) and used sensitivity analysis to identify key reaction steps leading to ignition. We added additional reactions of the key intermediates (peroxide radicals), and refined the rate estimates and thermochemistry using quantum mechanical calculations. These improvements to the models fixed some known problems in the models, but did not significantly improve the model's reliability for predicting ignition delays. We believe this is because the literature models were tweaked to reproduce all of the limited number of existing data; additional data will be required to assess whether any of these chemistry models is really predictive.

For computer-aided engine design, one needs to be able to rapidly assess how a proposed new design will work. This is quite challenging for HCCI strategies which rely on hot residuals, since one must simulate many engine cycles before the simulation reaches a steady state; at each cycle one must solve the full chemistry as well as doing several flow and transfer calculations. Using conventional solvers and methods (e.g. CHEMKIN) it takes more than 1 CPU-hour for each engine cycle, so it would take a week to simulate 6 seconds of engine run time at $1500 \mathrm{rpm}$. Note that this task is not easy to parallelize over multiple processors, since the computation is essentially sequential: the outcome of the first cycle simulations sets the initial conditions for the second cycle and so on. The conventional approach to deal with this is to reduce the complexity of the chemistry model. However, this model-reduction task is quite challenging when the original model contains $\sim 5000$ reactions and more than 1000 species. We therefore automated the model reduction process, writing a Web-accessible software package (RIOT) which performs the reduction to the user's error tolerances.

Although model-reduction is the conventional approach, we found we could obtain equally dramatic CPU-time reductions while retaining all the accuracy of the full-chemistry engine simulations by rewriting the governing equations in a way that made the chemistry Jacobian extremely sparse. We were then able to take advantage of this sparsity using software developed at MIT by our collaborators. This reduces the CPU time required for an HCCI engine simulation by 1-2 orders of magnitude, so we can run single-cycle simulations in a few minutes, 
and a 100-cycle full-chemistry transient simulation overnight on a PC. With this dramatic reduction in the CPU-time required for the chemistry calculations, we find that most of the CPU time is now used computing the flows in the engine; further improvements in computational efficiency and "model reduction" should be aimed at the fluid flow calculations rather than at the chemistry. For more information see Schuchardt, K., et al. (2005), Green (2004) and Green et al. (2004)

\section{Computation of HCCI engine behavior}

We have developed an HCCI engine simulator suitable for full-chemistry transient or steady-state simulations. As part of the simulator development we created new software for chemistry model reduction and fast algorithms for full-chemistry engine simulations. We have used the simulator primarily to understand what limits the operating range of HCCI, both at the high-load and low-load end of the range, and to understand the response of HCCI engines to transients in load, speed, air/fuel ratio, and the transients induced by switching between SI and HCCI modes. Our focus has been on engines which can switch between SI and HCCI modes, and which use negative valve overlap to provide the heat to sustain combustion. We have also studied the effects of fuel composition on the HCCI operating range, and we have used quantum chemistry to study several types of chemical reactions hypothesized to be important in HCCI engine operation.

The overall conclusions from this work:

1) For naturally aspirated HCCI engines, the high-load limit is usually controlled by "HCCI knock", loud operation correlated with high rates of pressure rise. This limit can be significantly raised by use of supercharging or turbocharging, but this can lead to very high peak pressures in the cylinder, and consequent materials limitations. In some situations, the peak load is also limited by misfire. Within the normal market gasoline range of fuels, the highload limit is not very fuel-sensitive.

2) The low-load limit is controlled by the difficulty in providing the heat needed to ignite fuel/air mixtures which have been heavily diluted with residual gases. Again, the low-load limit is not very sensitive to fuel variations within the range typical of market gasolines, particularly at high engine speeds. At low engine speeds operation is more sensitive to fuel composition.

3) One can make a good zero-order model for SI/HCCI engines using existing mass/heat flow models and approximate fuel (e.g. PRF) chemistry models. These models are sufficient for getting an initial understanding of the system. However, close scrutiny reveals many discrepancies between the model predictions and experiment that limit the utility of the models for design of either fuels or of engines.

4) Existing fuel chemistry models have many deficiencies (and they also do not model realistic fuel compositions). The MIT group has been working hard to improve the chemistry models, with limited success. Existing models for heat 
transfer and mass flows in engines are also not accurate enough to provide quantitative predictions of HCCI engine operation. In many cases, it is difficult to determine whether discrepancies between the simulator and experiment are due to errors in the simulations of heat/mass transport or to errors in the simulations of fuel chemistry.

For additional information see Angelos et al. (2007), Chesa Rocafort et al. (2005), Yelvington et al. (2003, 2004)

\section{Developing Reduced and Skeletal Kinetic Models (UCB)}

This goal of this project was to reduce chemical kinetic computational times significantly. It employed two approaches: the first developed new reduced and skeletal models for isooctane based on the accepted LLNL model of Curran, et al., (2002); the second approach used an Artificial Neural Network to develop extremely fast algorithms to compute ignition delay times for use in CFD and system models.

\section{Isooctane Skeletal Mechanisms Based on LLNL Detailed Mechanism}

Our previous development has led to a skeletal mechanism with 199 species and 383 steps (Aceves et al. 2002). The CPU time is reduced by roughly a factor of 25-30 for HCCI applications. A more accurate skeletal mechanism with 259 species was also developed and its performance is shown in Figure 3.1-3.2. Overall the agreement with LLNL detailed mechanism is satisfactory for temperatures above $850 \mathrm{~K}$. The skeletal mechanism predicts slightly longer ignition delays for low temperature regime below about $850 \mathrm{~K}$. The time saving on CPU time over LLNL is about a factor of 20-25 depending on the conditions.

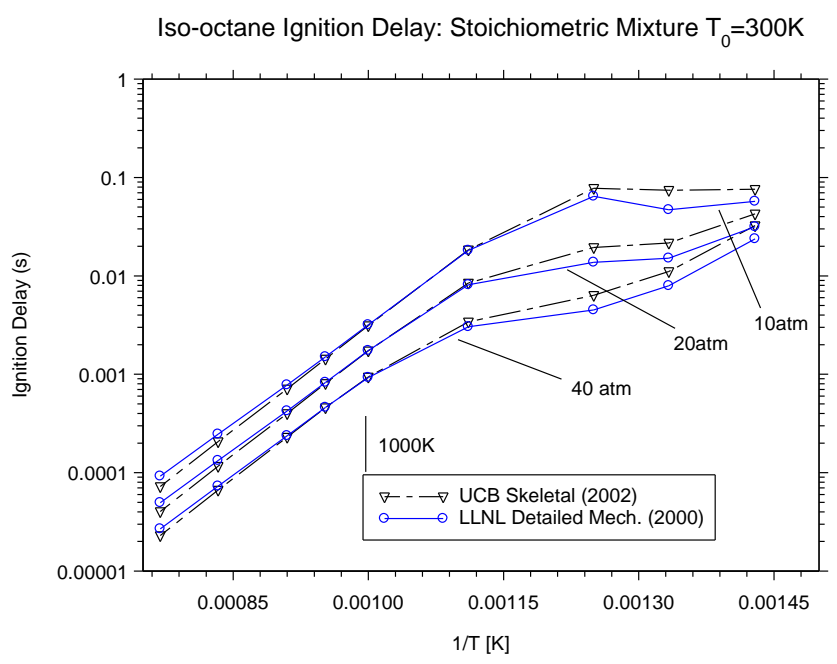

Figure 3.1: Comparisons of predicted ignition delay times for a constant volume time dependent reactor with a skeletal chemistry by University of California at Berkeley and detailed LLNL mechanisms. Conditions: stoichiometric isooctane and air mixture, initial temperature $300 \mathrm{~K}$, initial pressures: 10-40 atm. 


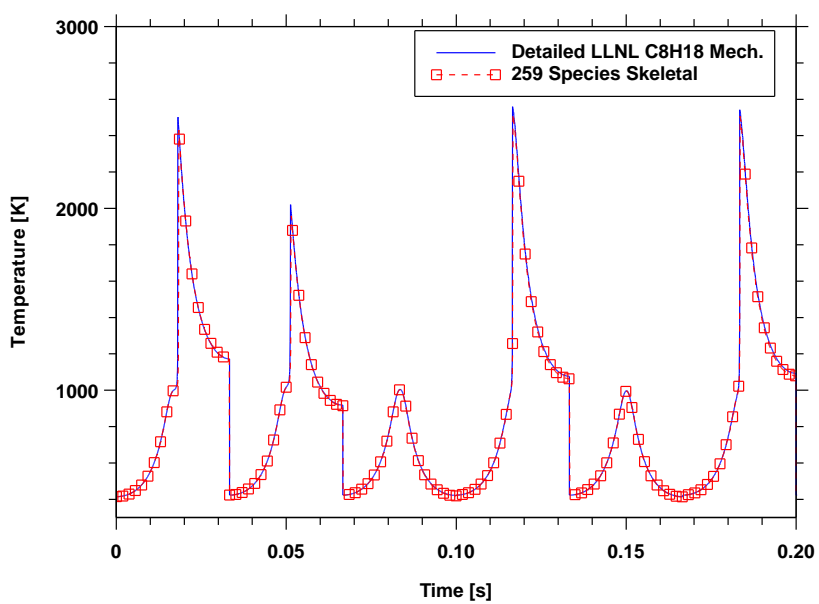

Fig. 3.2. Comparison of predicted results obtained from the detailed LLNL mechanism and the skeletal mechanism for isooctane with ignition followed by misfire. The simulation was conducted with a well-mixed reactor with stoichiometric isooctane-air mixture with exhaust gas

Recently a new reduced mechanism for Iso-octane has been developed using a QuasiSteady State Analysis (QSSA) Low Temperature Combustion (LTC) search algorithm. This search algorithm is directed by choosing operating points important to LTC combustion. This process reduces the number of species from the full LLNL mechanism (857) to a skeletal mechanism (215) and further down to a reduced mechanism of only 63 species. This mechanism was then validated by comparing autoignition times, transient well-mixed reactor temperatures and emissions, and Kiva3v. The validation runs in Kiva3v show a 6 fold reduction in running time when comparing the reduced (63) and skeletal (215) mechanisms. The 63 species reduced chemistry for isooctane developed in last quarter has been implemented into the Kiva3v computer code supported by Los Alamos National Laboratory (Chen et al., 2007 and Bisetti et al., 2007).

\section{ANN model for ignition delay}

An artificial neural network (ANN) based combustion model has been developed and has been integrated it into a fluid mechanics code (KIVA3V) to produce a new analysis tool (titled KIVA3V-ANN) that can yield accurate HCCI predictions at very low computational cost (Choi and Chen, 2005 and Aceves et al., 2007). The neural network predicts ignition delay as a function of operating parameters (temperature, pressure equivalence ratio and residual gas fraction). KIVA3VANN keeps track of the time history of the ignition delay during the engine cycle to evaluate the ignition integral and predict ignition for each computational cell. After a cell ignites, chemistry becomes active, and a two-step chemical kinetic mechanism predicts composition and heat generation in the ignited cells. KIVA3V-ANN has been validated by comparison with isooctane HCCI experiments in two different engines. The neural network provides reasonable predictions for HCCI combustion and emissions that, although typically not as good as obtained with the more physically representative multi-zone model, are obtained at a 
much reduced computational cost. KIVA3V-ANN can perform reasonably accurate HCCI calculations while requiring only $10 \%$ more computational effort than a motored KIVA3V run. It is therefore considered a valuable tool for evaluation of engine maps or other performance analysis tasks requiring multiple individual runs.

\section{Experimental Verification of Chemical Mechanisms (UM and Stanford)}

\section{Lean Iso-octane/Air Ignition Delay Time Measurements and Modeling}

To investigate the ignition delay time behavior at extreme lean HCCI operation, ignition delay time measurements were performed at low equivalence ratios using the Rapid Compression Facility (RCF) at the UM and the High-Pressure Shock Tube Facility (HPSTF) at SU. Complementary experimental regimes relevant to HCCI engines (with an emphasis on lean operation) were studied using the two devices.

Ignition times for lean $(\phi=0.25)$ iso-octane/air mixtures were measured behind reflected shock waves at the Stanford high-pressure shock tube facility (high-purity, unheated, $5 \mathrm{~cm}$ diameter, helium-driven, turbo molecular pumped shock tube). In the current work, ignition times were measured using sidewall $\mathrm{OH}^{*}$ emissions. Due to the lean conditions of these experiments and gradual pressure rise before ignition, pressure is not a good indicator for quantitative measurements of ignition times. Shock wave experiments were performed for a wide range of temperatures and pressures: 1021-1302 K and 21-51 atm. Measurements for the same fuel at higher $\phi$ values have previously been conducted by Davidson et al., (2004) under this contract. The results of the two studies are summarized in Fig. 3.3.

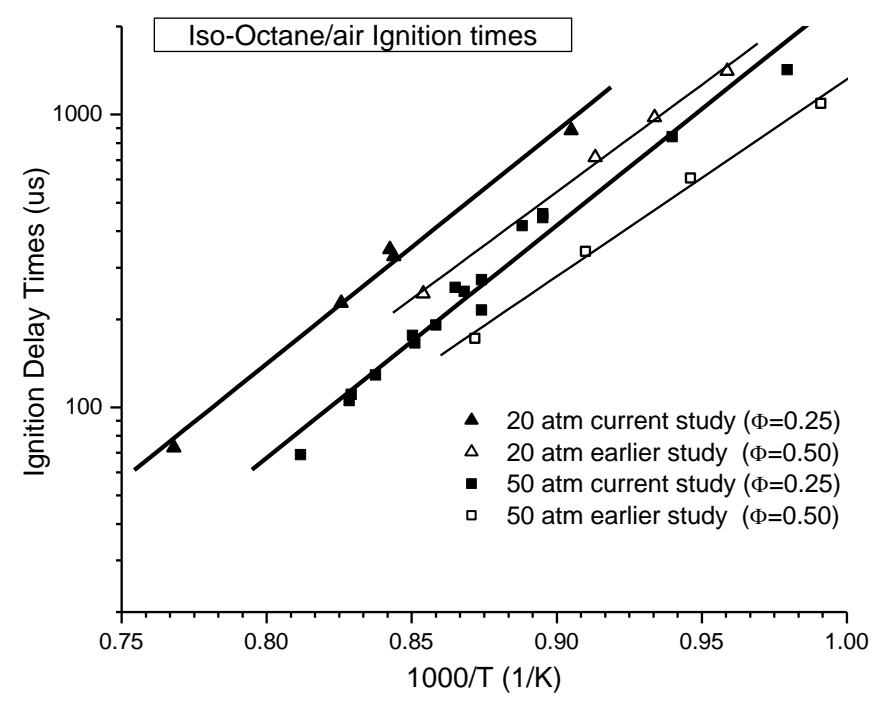

Fig. 3.3. Iso-octane/air ignition delay time for different $\Phi$ and pressure values. 


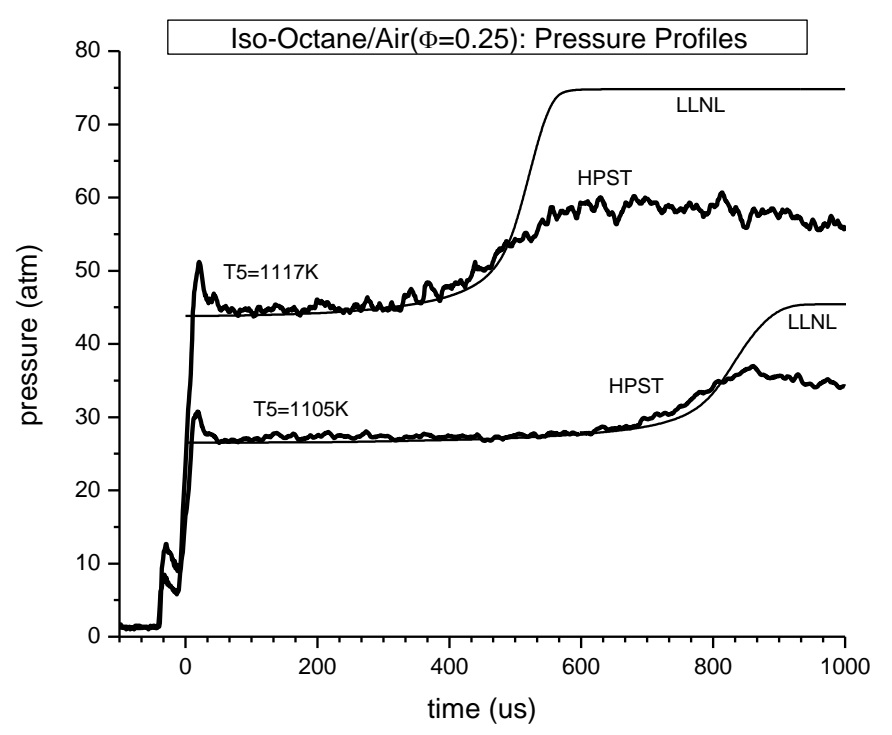

Fig. 3.4. Iso-octane/air experimental pressure profiles with LLNL model.

Pressure time-history profiles show significant pre-ignition energy release. Example pressure traces are presented in Fig. 3.4. Very little $\mathrm{CH}^{*}$ emission occurred in the lean experiments when compared to the significant emission seen in the $\phi=1$ study.

Regression analysis shows ignition time correlation with pressure, temperature and $\phi$ as over the conditions of the current and previous SU studies as:

$$
\tau_{\text {ign }}(u s)=0.0722 \times P(\text { atm })^{-0.90} \times \phi^{-0.60} \times \exp [12370 / T(K)]
$$

Modeling of the ignition process was conducted using several different mechanisms including those of LLNL (Curran et al., 2002) and Chalmers University (Ogink and Golovitchev). A comparison of the model predictions using the LLNL mechanism and the measured pressure time-history profiles are show in Fig. 3.4. The LLNL mechanism successfully models the pressure traces over the portion of the ignition where the shock tube is behaving like a constant volume reactor. Rate of production and sensitivity analysis work comparing the reaction pathways and identifying key species for iso-octane oxidation at various equivalence ratios is currently underway at SU and UM.

Ignition delay time measurements were conducted in the UM RCF over a range of conditions ratios $(\phi=0.2-1.0)$, pressures $(P=5.1-23$ atm $)$, temperatures $(T=943-1047 \mathrm{~K})$, oxygen mole fractions $\left(\chi_{\mathrm{O} 2}=9-21 \%\right)$, and with the addition of trace amounts of combustion product gases $\left(\mathrm{CO}_{2}\right.$ and $\left.\mathrm{H}_{2} \mathrm{O}\right)$. Pressure was used to determine the ignition delay time, as well emission time histories. The ignition delay time determined using the two measurements were found to be in excellent agreement throughout the range of conditions studied.

In order to provide a more rigorous benchmark for the kinetics modeling, absolute quantitative $\mathrm{OH}$ absorption time-histories have also been obtained in UM RCF studies over a 
range of experimental conditions. Typical results are shown in Fig. 3.5. The peak in the $\mathrm{OH}$ time- histories systematically occurs after the ignition (as defined based on pressure).
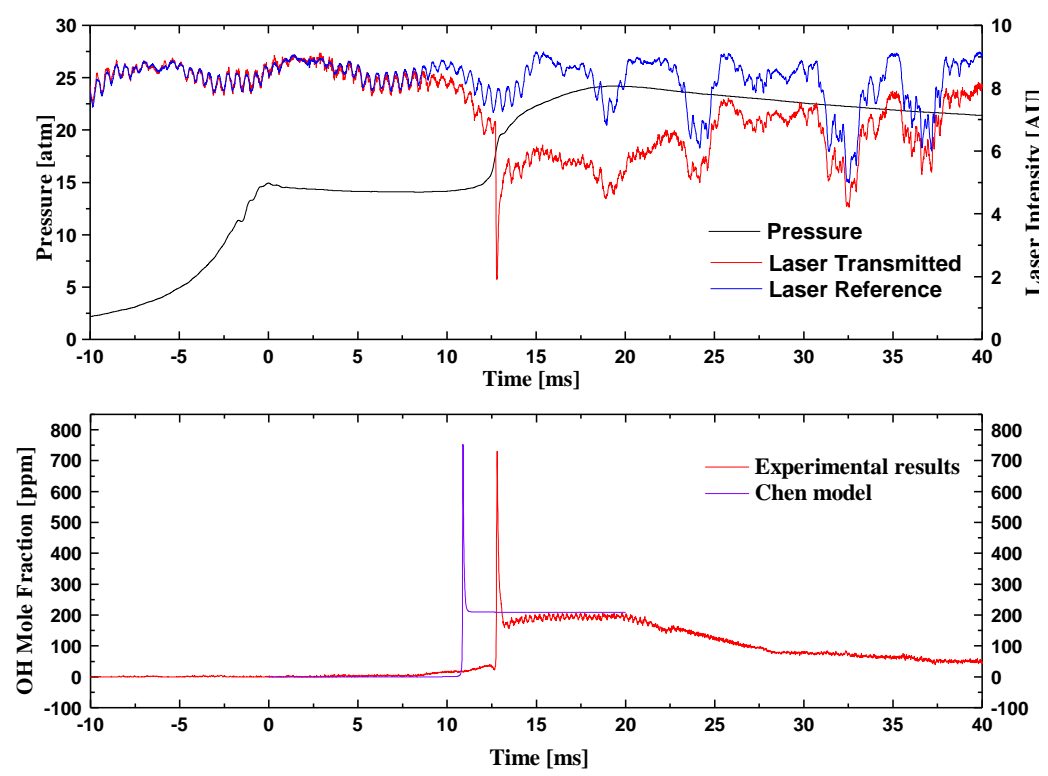

Fig. 3.5. OH mole fraction time-history obtained using differential laser absorption spectroscopy in an isooctane ignition experiment conducted in the UM RCF. The experimental conditions are provided in the figure.

The performance of several isooctane reaction mechanisms at reproducing the experimentally measured $\mathrm{OH}$ mole fraction profiles are shown in Fig. 3.6.
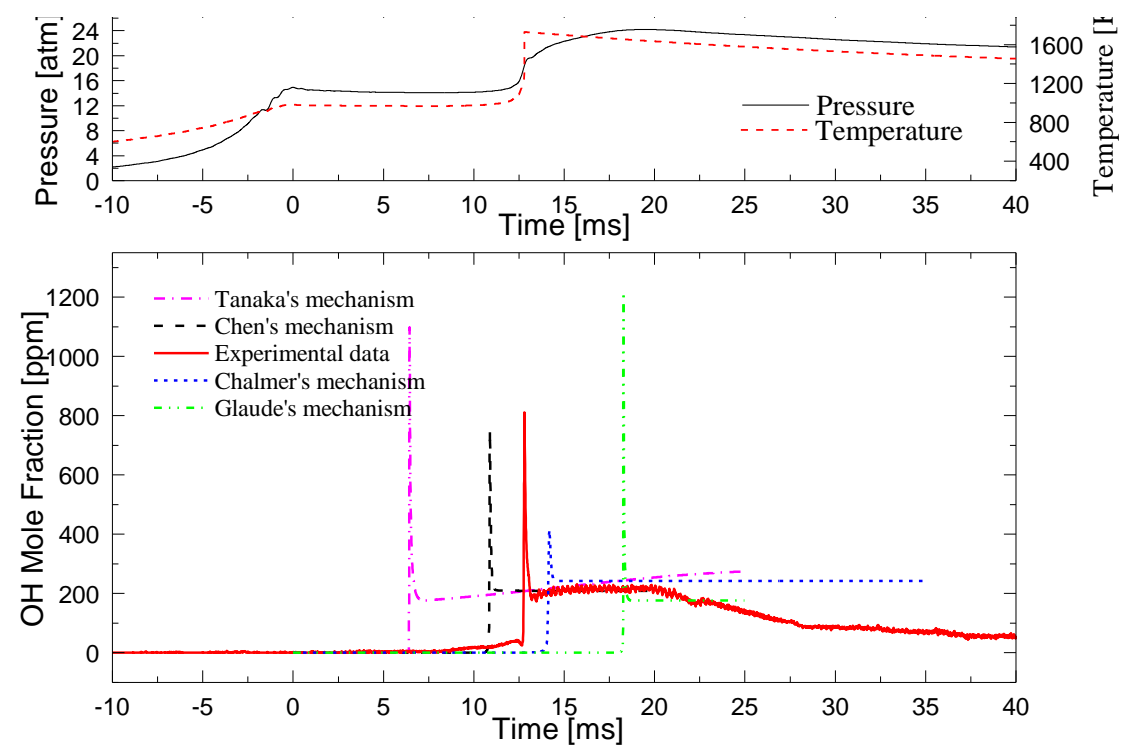

Fig. 3.6 Comparison of measured $\mathrm{OH}$ mole fraction data of Fig. 3.5 with predicted $\mathrm{OH}$ mole fraction time-histories using various isooctane mechanisms. The UM RCF experimental conditions are provided in the figure. 
Additionally, sensitivity analysis has been conducted to identify the reactions with the greatest impact on the $\mathrm{OH}$ time-histories. Corresponding sensitivity and rate of production results corresponding to the $\mathrm{OH}$ data shown in Fig. 3.5 are shown in Figs. 3.7 and 3.8.

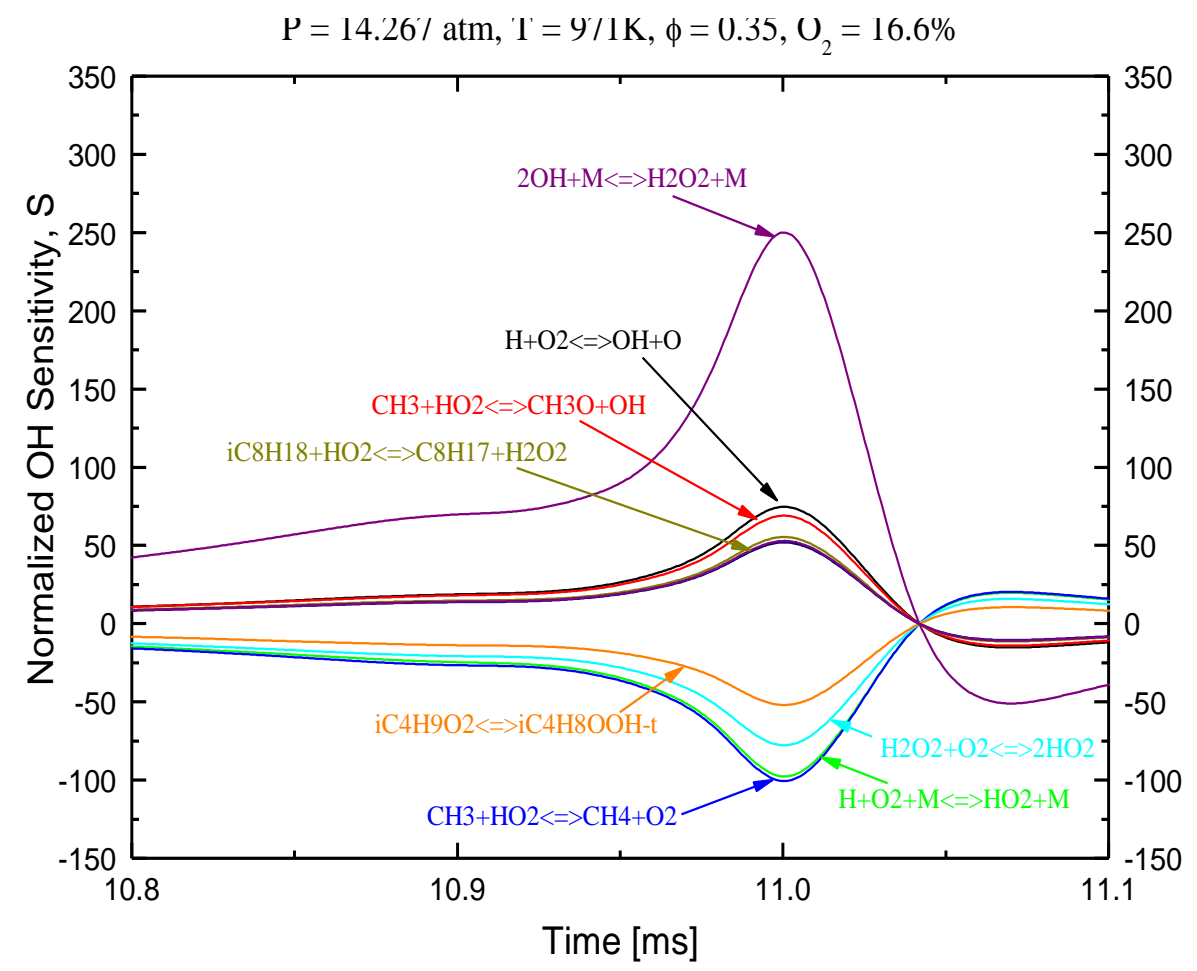

Fig. 3.7. $\mathrm{OH}$ sensitivity analysis corresponding to the UM RCF isooctane ignition data shown in Fig. 3.5.

The sensitivity data are critical for assessing the relative importance of the detailed reactions represented in the models. As seen in Fig. 3.7, the sensitivity analysis indicates the importance of the hydroxyl self-reaction (recombination) to form hydrogen peroxide at these lean conditions. Sensitivity analysis at higher equivalence ratios shows increased importance of the carbon chemistry in affecting $\mathrm{OH}$ profiles. By identifying the key reactions, the sensitivity data also provide a means to assess the uncertainty in the predictive capabilities of the detailed isooctane reaction mechanisms attributable to the uncertainties in the individual rate coefficient data used in the various isooctane mechanisms. 


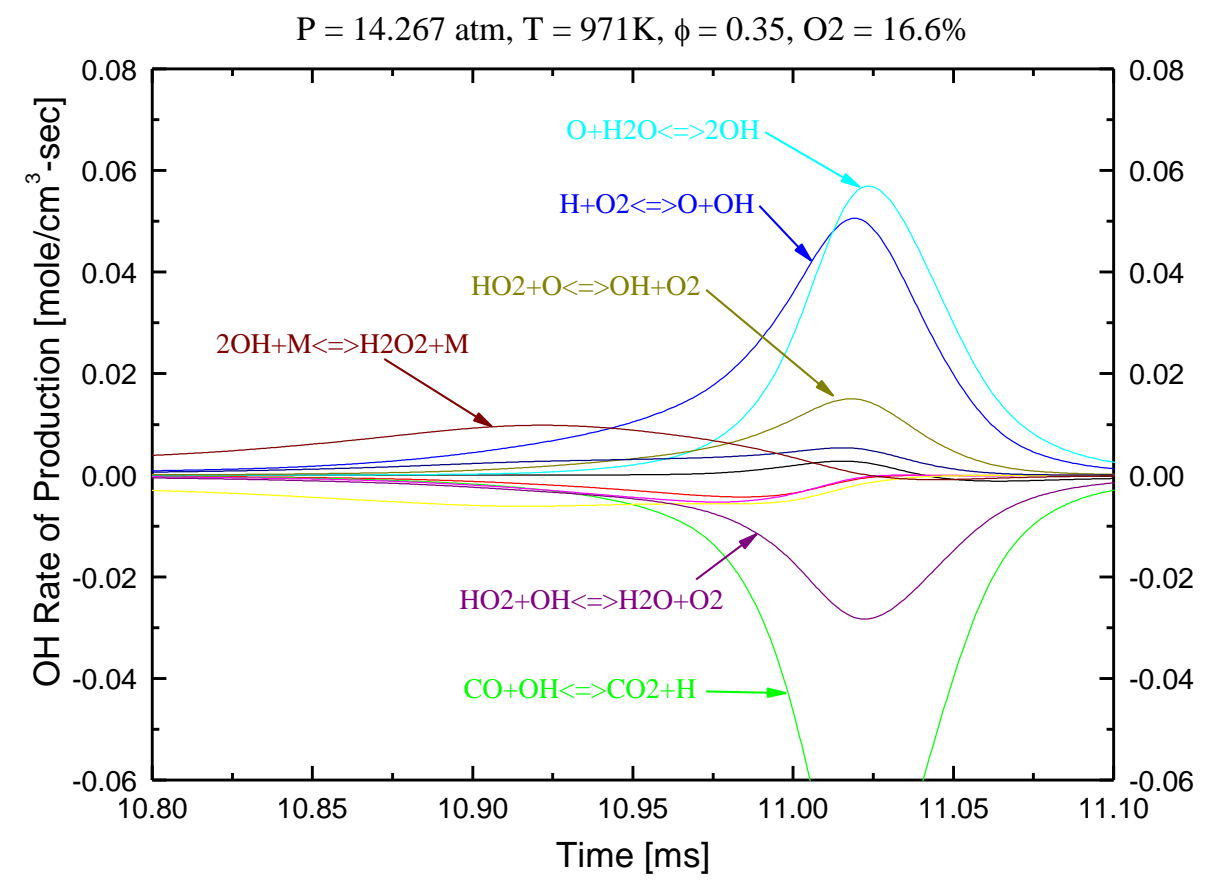

Fig. 3.8 OH rates of production corresponding to the UM RCF isooctane ignition data shown in Fig. 3.5. 


\section{Ignition Delay Time Correlation}

A summary of the $\tau_{\text {ign }}$ data from the UM RCF and the SU HPSTF studies is shown in Arrhenius coordinates in Fig. 3.9. When the data from both studies (excluding the SU $\phi=1.0$ data) are considered, the following expression for $\tau_{\text {ign }}(\mathrm{ms})$ was obtained:

$$
\tau_{\text {ign }}(m s)=2.385 \times 10^{-4} \cdot P^{-1.0} \cdot \phi^{-0.83} \cdot \chi_{O_{2}}^{-1.51} \cdot \exp \left(32700 / R_{[\text {cal } / \mathrm{mol} / K]} T\right)
$$

Additional UM RCF studies are currently underway investigating additional fuels of interest including real fuels.

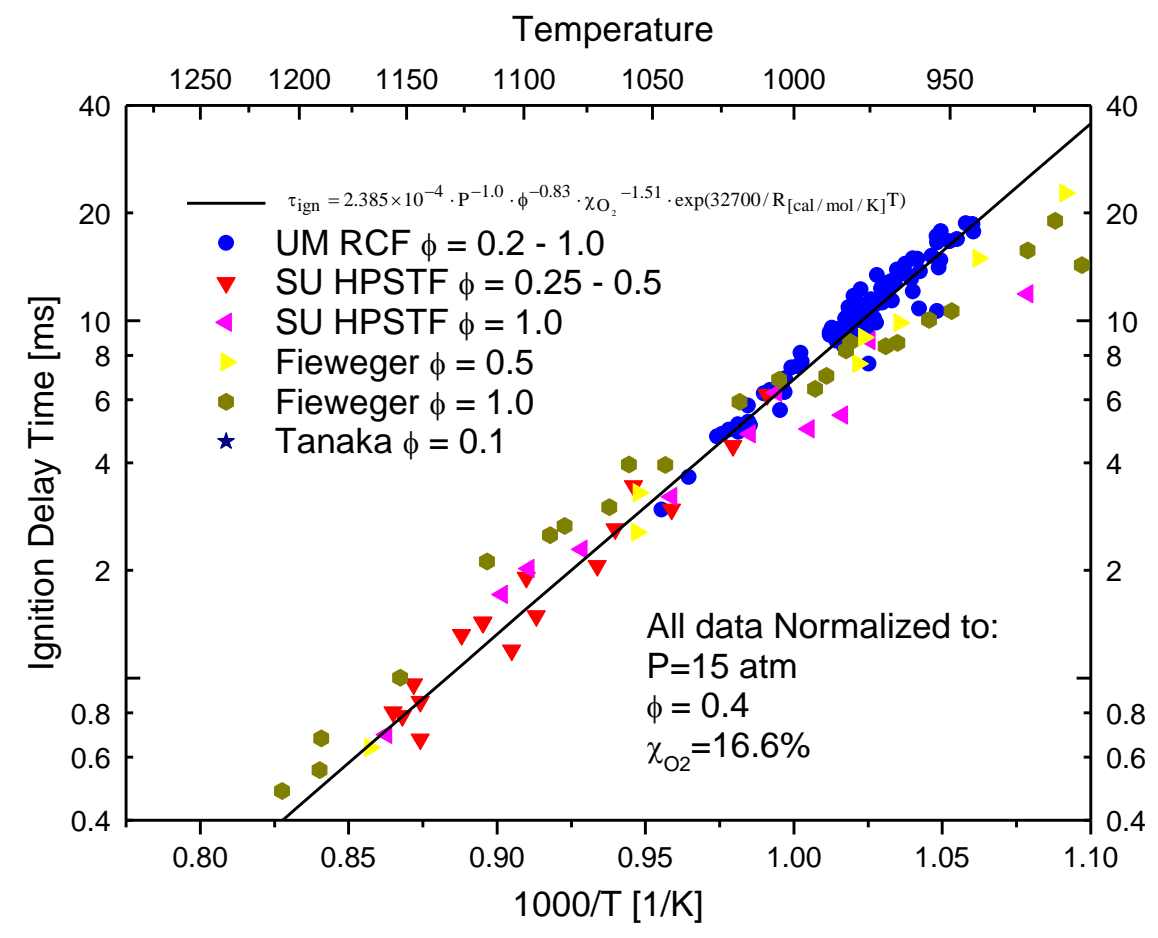

Fig. 3.9. Summary of UM and SU experimental data on $\tau_{\text {ign }}$ for isooctane. Data from other studies are also included $\mathrm{f} 4.3 \mathrm{a}$ Computational and Experimental Verification of Chemical Mechanisms (UM)

\section{Reaction Front Propagation}

Additional UM RCF experiments conducted to obtain high-speed imaging data indicate that the concentration of $\mathrm{O}_{2}$ is a key parameter that indicates the ability to sustain a reaction front in the mixtures. For example, Figs. 3.10 and 3.11 show time sequences of conditions where reaction front propagation cannot be sustained (Fig. 3.10) and where reaction from propagation sustained (Fig. 3.11). 


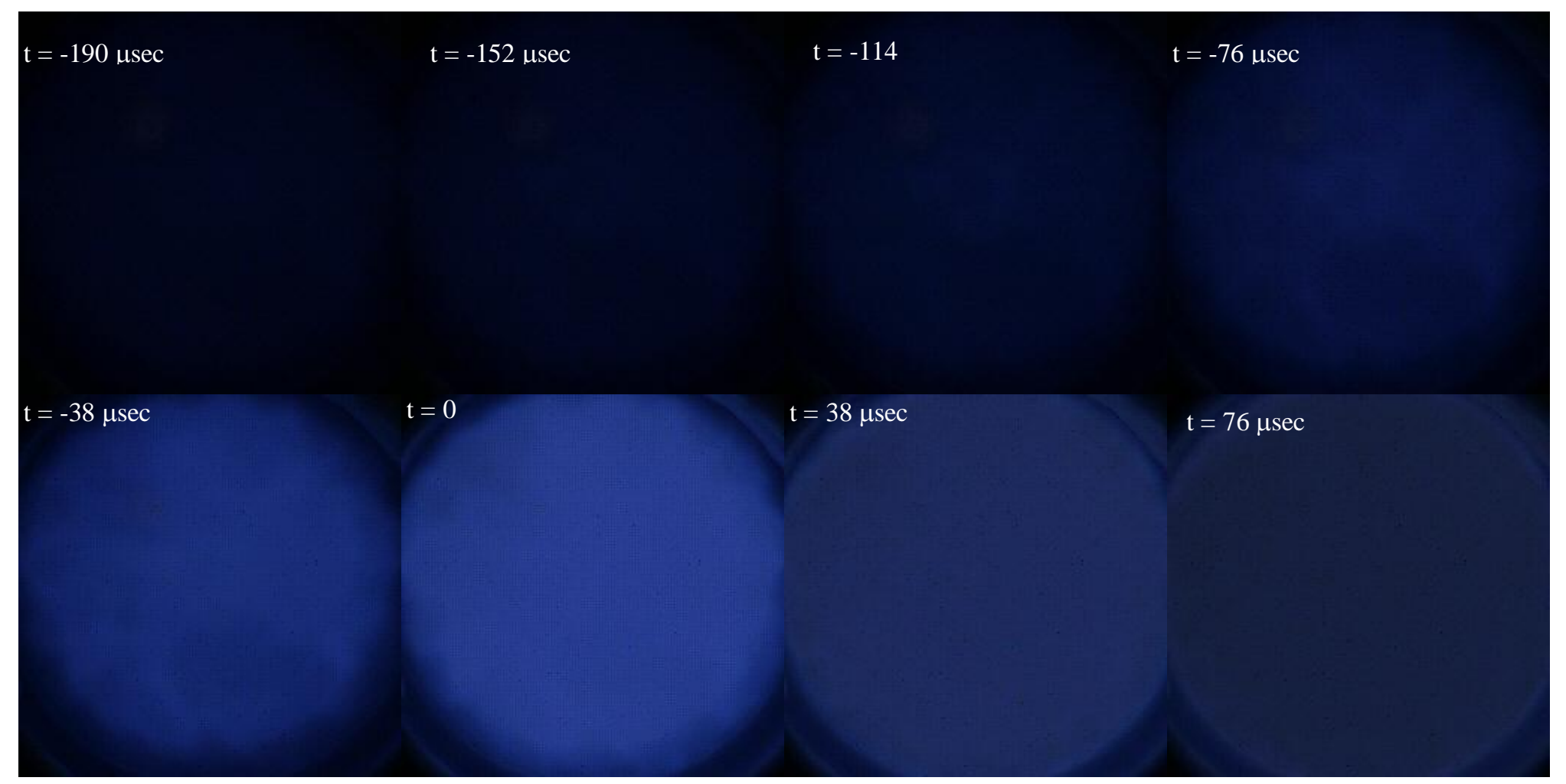

Fig. 3.10. Reaction front not sustained. High-speed digital imaging sequence obtained in RCF studies of isooctane/air ignition, experimental conditions of $\phi=0.3, \mathrm{~T}_{\text {eff }}=1015 \mathrm{~K}, \mathrm{P}_{\text {eff }}=8.8 \mathrm{~atm}$, inert gas: $\mathrm{O}_{2}$ ratio $=5$, sample acquisition rate of 26,000 frames per second $(38.5 \mu \mathrm{s}), 33 \mu \mathrm{s}$ exposure time. 


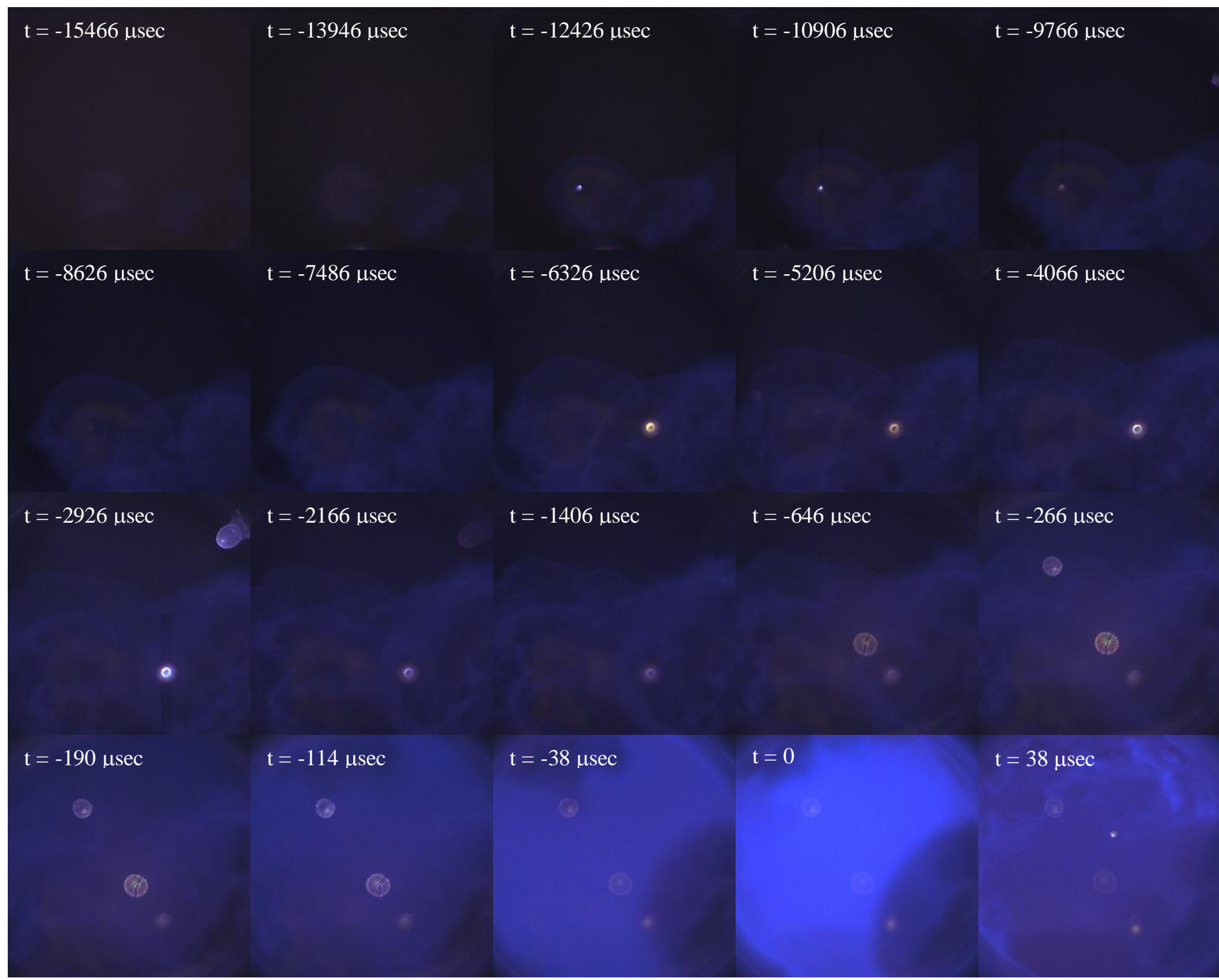

Fig. 3.11 Reaction front sustained. High-speed digital imaging sequence obtained in RCF studies of isooctane/air ignition, experimental conditions of $\phi=0.2, \mathrm{~T}_{\text {eff }}=903 \mathrm{~K}, \mathrm{P}_{\text {eff }}=149 \mathrm{psi}$, inert gas: $\mathrm{O}_{2}$ ratio $=1.38$, sample acquisition rate of 26,000 frames per second ( $\left.38.5 \mu \mathrm{s}\right), 20$ $\mu$ s exposure time. 


\section{Investigation of chemical kinetics for gasoline HCCI}

Ignition delay measurements were carried out in the UM Rapid Compression Facility (RCF) and in shock tubes at Stanford. These included gasoline and gasoline surrogates in addition to isooctane. Figure 3.12 shows shock tube results from Stanford (Gauthier, et al. 2004) comparing gasoline and a ternary mixture of isooctane/toluene/n-heptane $(63 / 20 / 17)$ by vol. \% which represents gasoline well. These findings provide a comprehensive benchmark to be used by the modeling community to assess and construct detailed kinetic mechanisms to represent gasoline.

The work also revealed that gasoline ignition delays are similar to those of isooctane. Further, the simple autoignition correlation developed at UM for isooctane, represents both isooctane and gasoline well over most of the operating zone of HCCI engines. Figure 3.13 shows the ignition delay correlation of He et al., (2005) (straight lines) compared to the data for both gasoline and isooctane. Except for the lowest temperatures and equivalence ratio of 1.0 the agreement is excellent. This means that practical use of the ignition delay correlation should be broadly applicable to gasoline for use in the GT-Power system model discussed above.

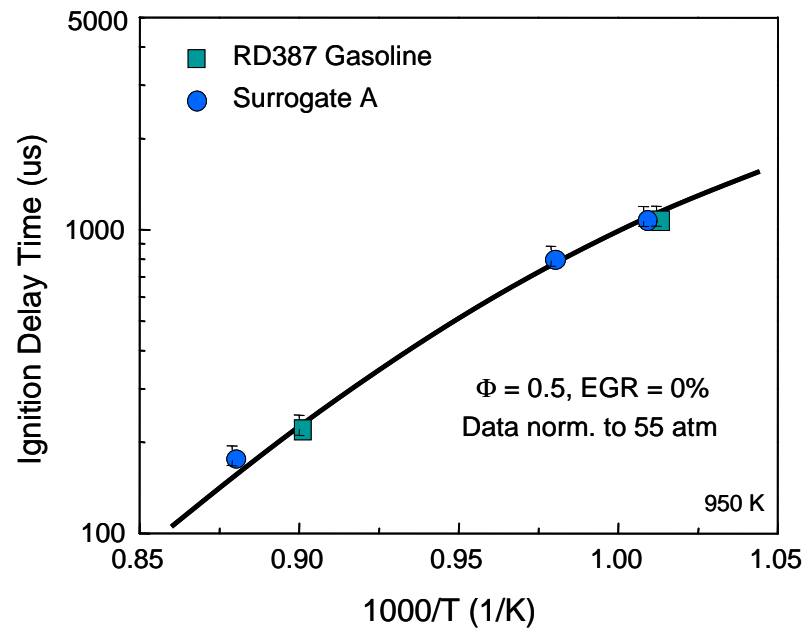

Fig. 3.12. Shock tube ignition delay measurements showing good agreement between gasoline and a proposed surrogate ternary mixture made up of isooctane/toluene/n-heptane: 63/20/17 vol. \% (Stanford).

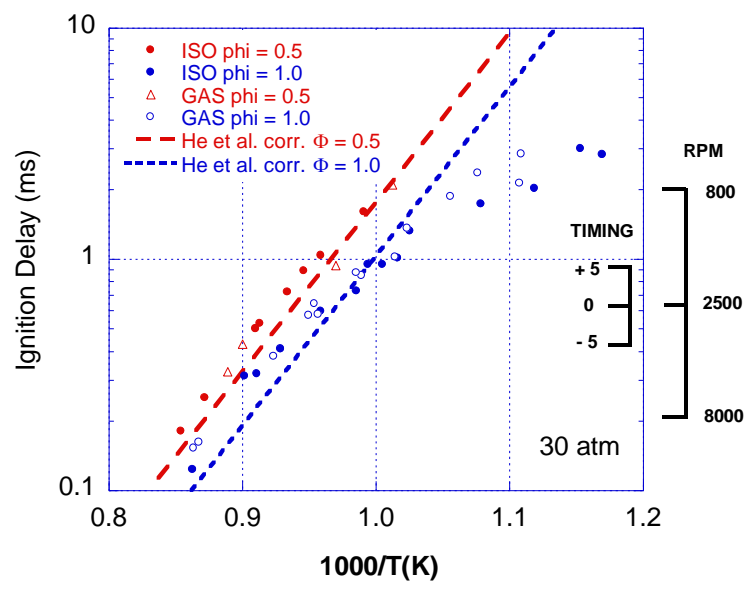

Fig. 3.13. Shock tube ignition delay data for gasoline (triangles) and isooctane (circles) for equivalence ratios 0.5 and 1.0. Dashed lines show the auto-ignition delay correlation for isooctane. (He et al., 2005). Scale on right indicates approximate delay times for a range of engine speed and combustion timing (Stanford, UM). 


\section{Using $\mathrm{H} 2$ and $\mathrm{CO}$ from Gasoline Reforming to Control the Auto-Ignition of Fuel-Air Mixture (MIT)}

The task is to examine the potential of using hydrogen, and hydrogen plus $\mathrm{CO}$ produced in a gasoline reformer, such as the plasmatron, to control the autoignition characteristics of the fuel-air mixture. Based on this analysis, hydrogen-assisted HCCI/gasoline engine concepts were developed and assessed. One promising concept is to use the octane enhancing behavior of hydrogen plus $\mathrm{CO}$ to improve knock resistance in the spark-ignition combustion mode and thereby allow a lower octane base hydrocarbon fuel to be used in the HCCI combustion mode.

Two chemical autoignition models, one developed at MIT in this DOE HCCI engine program, and the other at Lawrence Livermore Laboratory, have and are being used to examine the impacts of $\mathrm{H}_{2}$, and $\mathrm{H}_{2} \mathrm{CO}$ mixtures, on the autoignition process for different base primary reference fuels. An engine simulation model which can explore how best to combine lower octane easier-to-ignite base fuels for the HCCI operating mode, with the $\mathrm{H}_{2}$ and $\mathrm{CO}$ enhancedoctane base fuel in the spark-ignition operating mode, is being used to define in-cylinder requirements. These are the mixture conditions required to achieve controlled autoignition with a range of base fuels, and the corresponding intake temperatures and residual fractions needed to obtain these conditions. Also experiments in an existing Sloan Automotive Lab rapid compression machine designed to validate these kinetic calculations are planned. A computer code which connects the chemistry models to the in-cylinder rapid compression machine conditions is available from a prior study, and the modifications needed to use this code in this study are being made. 


\title{
Task 4: Detailed Modeling of Reaction, Mixing and Spray Dynamics Using CFD Codes and Experimental Validation
}

\author{
Detailed CFD Modeling of Combustion and Mixing and Experimental \\ Validation (UM)
}

\section{KIVA-RIF Implementation}

In an effort to develop the ignition/combustion modeling in KIVA $3 \mathrm{~V}$ based on the representative interactive flamelet (RIF) model, the mixture fraction and its variance equations have been successfully implemented and tested. The flamelet calculation will be done using Flame Master (Pitsch et al., 1996), which has been modified for the present HCCI combustion situations. In particular, the conditional cell-averaged scalar dissipation rate, $\langle\tilde{\chi} \mid \tilde{Z}\rangle$, needs to be parameterized as an input to the flamelet solver. This is done by fitting the statistical distribution obtained by the KIVA calculation with a parabolic function.

\section{KIVA-RIF-LES}

While the above flamelet approach is applied to conventional RANS turbulence modeling, RIF is applicable to more sophisticated turbulence models such as large eddy simulation (LES). Therefore, in parallel with the above approach, the turbulence model part of the existing KIVA 3V code is modified to implement the LES capability. The LES model is based on a previous work at UM (Papageorgakis, 1997) where a dynamic subgrid model following the approach of Germano (1991) has been employed. This approach has an advantage over the commonly-used Smagorinsky model by determining the subgrid model constant dynamically as a solution procedure, thereby eliminating an ad-hoc nature of the modeling. The previous LES effort at UM has been implemented to an earlier KIVA-3 version, hence it is necessary to integrate the model with the latest KIVA-3V which includes the valve motion.

\section{Parameterization of Ignition using a Counterflow Model}

As an alternative and complementary approach to the full KIVA simulation approach, a relatively simple parameterization of the ignition events in HCCI engines is proposed. A onedimensional counterflow model is adopted to investigate the effects of the key physical parameters on the ignition in an HCCI-like environment. This configuration consists of two opposing nozzles with premixed fuel/air charge flowing through one and hotter exhaust products through the other. This model can describe the effect of inhomogeneity in the cylinder due to insufficient mixing between the residual exhaust gases and the fresh charge. The model can be easily adapted to study the effects of composition inhomogeneities by modifying the boundary conditions. The mixing layer formed between these two streams can be characterized by a mixture fraction $(Z)$ and the scalar dissipation rate $(\chi)$, which represent the "mixedness" and the "rate of mixing," respectively. The mixture fraction is defined such that it varies from 1 in the fresh reactants to 0 in the hot exhaust gases. The ignition and propagation of reaction through the mixture can then be parameterized in terms of these two variables. This forms the basic framework of the flamelet approach, in which the two parameters are computed using KIVA and provides a means to predict corresponding ignition delays. 
As a preliminary study (Sankaran et al., 2003) the counterflow model was used to simulate the effect of EGR mixing on the ignition of methane-air mixture. The ignition delay of different mixtures characterized by $Z$ was computed at various nozzle velocities (which represent a $\chi$ variation). The results are shown in Figure 4.1 along with a zerodimensional model for the ignition of those mixtures. In general, the ignition delay is shorter at the region close to the exhaust stream due to the higher temperatures of the exhaust gas. Moreover, mixing is found to affect ignition significantly at higher values of $\mathrm{Z}$, where the 0 -D model predicts much slower ignition delays. Upon systematic calculations, the results can be summarized as shown in Figure 4.2 where the ignition delay of a typical HCCI system is mapped out in terms of the two parameters, $Z$ and $\chi$. An important implication of this study is that the ignition in HCCI engines can exhibit two distinct modes: (a) a spontaneous, chemically-driven homogeneous explosion mode and (b) a front propagation mode assisted by heat and radical diffusion. The transition between the two ignition modes was computed at various mixing rates as indicated by the dashed line in Figure 4.2. The results demonstrate that a significant portion of the mixture within the HCCI engine can ignite through a front propagation mode, especially for higher scalar dissipation rate as encountered in the presence of strong turbulence. Further studies are being undertaken with iso-octane chemistry to study the behavior of gasoline HCCI ignition under similar conditions. It is anticipated that the $(Z, \chi)$ parameterization approach can serve as an improved submodel for the multi-zone approach, which is discussed in the next subsection, by incorporating the effect of the mixing rate on ignition.

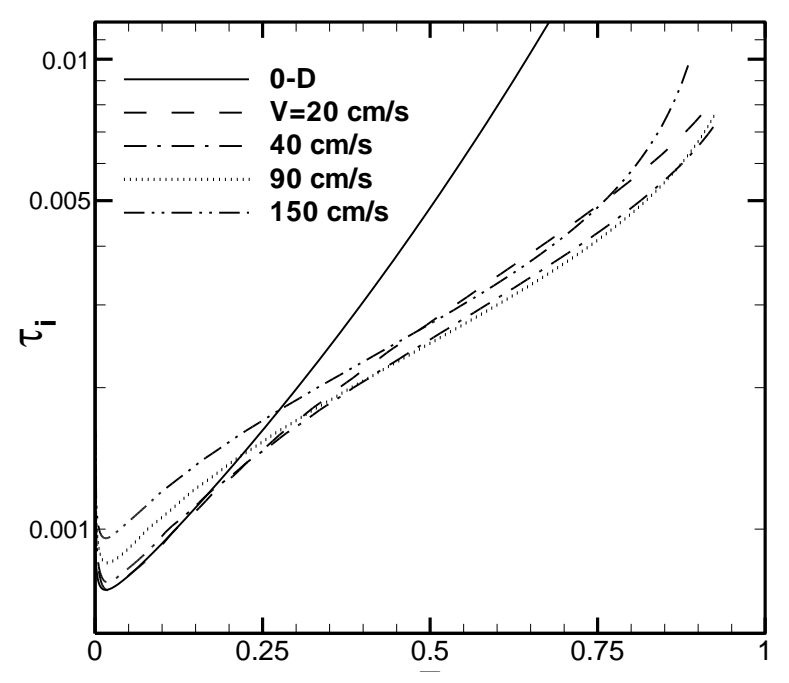

Fig. 4.1.Ignition time as a function of $\mathrm{Z}$ at increasing nozzle velocities.

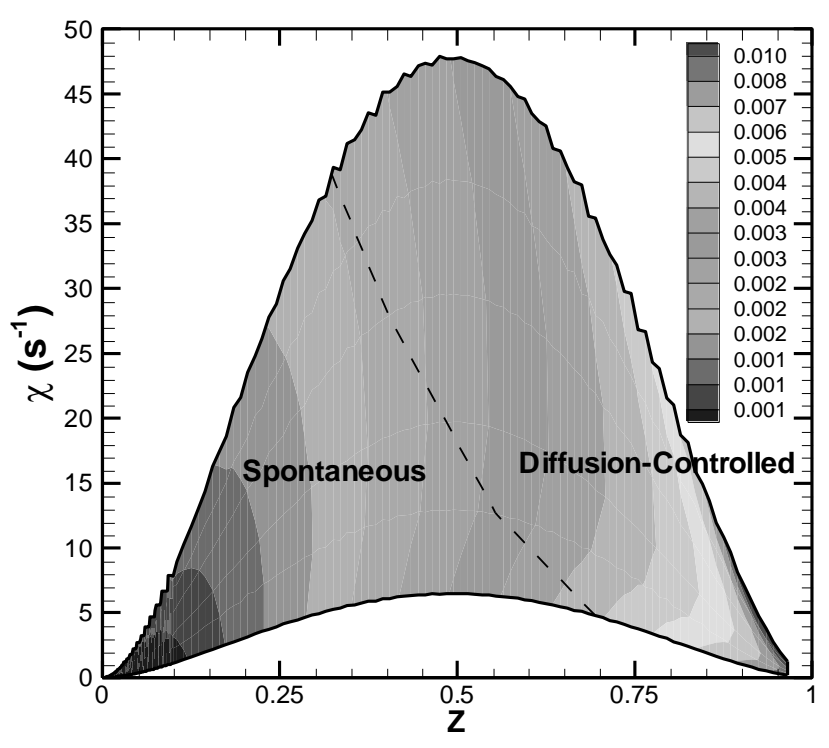

Fig. 4.2 Parametric mapping of ignition delay time (in s) as a function of local mixture composition and variance. 


\section{Effects of Non-uniform Temperature Distribution}

It is important to identify the operating condition maps of the HCCI engines over a wide range of parameters. However, due to the costs involved with such a wide range of parametric mapping, most of the previous studies were based on a singlezone model, hence did not account for the effects of stratification in a scalar variable. Therefore, a simple analytical model is developed to provide insights as to the effects of the statistical variations in the temperature distribution on the overall burn duration in the HCCI engines.

The theoretical model is constructed as follows. Consider a homogeneous mixture of reactants in the presence of turbulent temperature inhomogeneity represented by

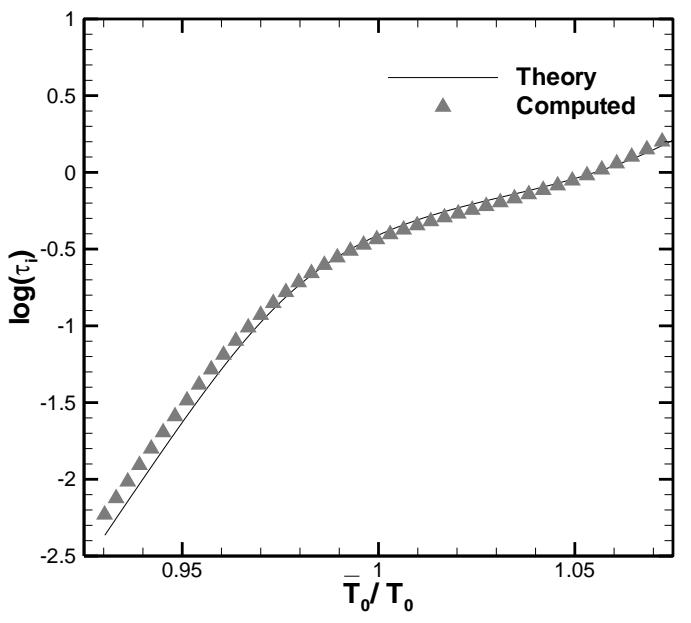

Fig 4.3. Ignition delay at various points in a stratified mixture as a function of the local temperature' the Gaussian distribution, with the mean and variance being the parameters. In this model, any given point in space is described by a homogeneous ignition without mixing and transport, yet the ignition is affected by the compression heating arising from the neighboring gases at different initial conditions. Assuming a single-step overall reaction, an analytic expression for the ignition delay can be described in terms of the initial temperature by the asymptotic analysis based on the entropy variable formulation of Linan and Williams (1995). Alternatively, a prescribed temperature pdf can be subdivided into a number of zones, and the ignition of these zones can be computed based on a multi-zone model calculation, where the pressure coupling between the zones due to compression heating was taken into account. Figure 4.3 shows the ignition delay as a function of the local temperature within the mixture with a Gaussian temperature distribution with $T^{\prime} / \bar{T}=$ 0.025 and $\left(P_{\text {final }}-P_{\text {initial }}\right) / P_{\text {final }}=0.5$. The asymptotic relation shows a very good agreement with the multi-zone model prediction. Therefore, it is confirmed that the ignition behavior can be described by the simple analytic model. The bending of the ignition delay variation shown in Fig. 4.3 represents that ignition of hotter spots in the mixture is retarded because they have to "heat up" the neighboring colder mixture pockets that will ignite subsequently.

The analytic results allow extensive parametric studies to produce various maps of operating conditions at very low cost. For example, the overall burn duration as a function of the variance of temperature fluctuation $\left(T^{\prime}\right)$ can be analyzed. Since different variance results in different ignition timing (CAD), for a fair comparison it is necessary to adjust the mean temperature as well so that the ignition pressure rise occurs always at top dead center. This involves a highly iterative process, such that even the multi-zone model calculation can be excessive for the analysis. 
Figure 4.4 shows the compiled result of the burn duration as a function of the variance of temperature fluctuation. It is clearly seen that the duration of burning increases continuously with an increase in the temperature nonuniformity, thereby potentially achieving smooth combustion in the HCCI engine. If $T^{\prime}$ becomes too large, however, excessively long duration of burning may result in a significant amount of cold end gas that is left unburned. Therefore, there must be a cut-off value of the maximum temperature variance.

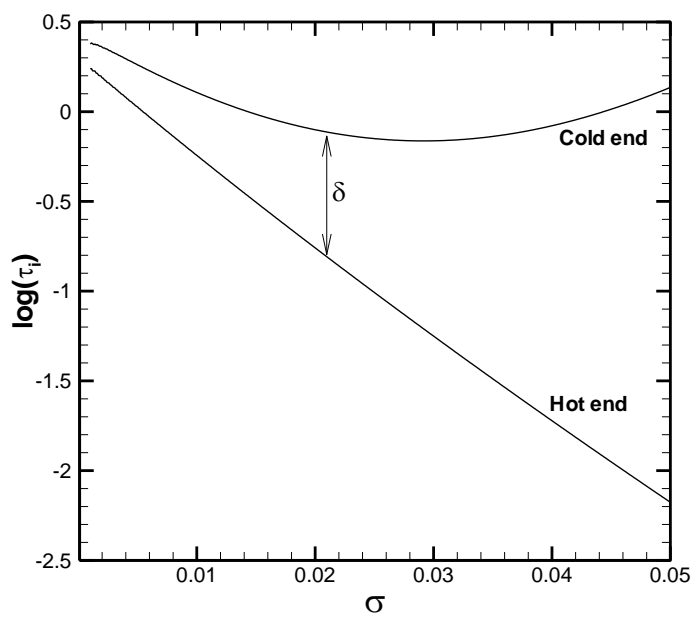

Figure 4.4 Duration of burning as a function of the variance in the temperature fluctuation based on the analytic model. At different values of the variance, the mean temperature has been adjusted such that the abrupt pressure rise occurs at TDC. 


\section{Large Scale Mixing of Residual Gases}

In addition to the flamelet work, we have carried out studies of large scale mixing of residual gases for an engine operated on premixed natural gas / air (Babajimopoulos et al., 2002, 2003). Based on an open cycle analysis with KIVA-3V it was found that at $30^{\circ} \mathrm{BTDC}$ significant variations in residual content were found in addition to the already well know temperature variations.

Figure 4.5 shows the resulting distribution of temperature - fuel/O2 equivalence ratio predicted by KIVA at $30^{\circ}$ BTDC for a case with negative overlap from $-60^{\circ}$ to $60^{\circ}$ ATDC intake. This results in a large quantity of EGR $(42 \%)$ so that the equivalence ratio is significantly lower than the supplied equivalence ratio of 0.3. As indicated in the figure, there is a large spread
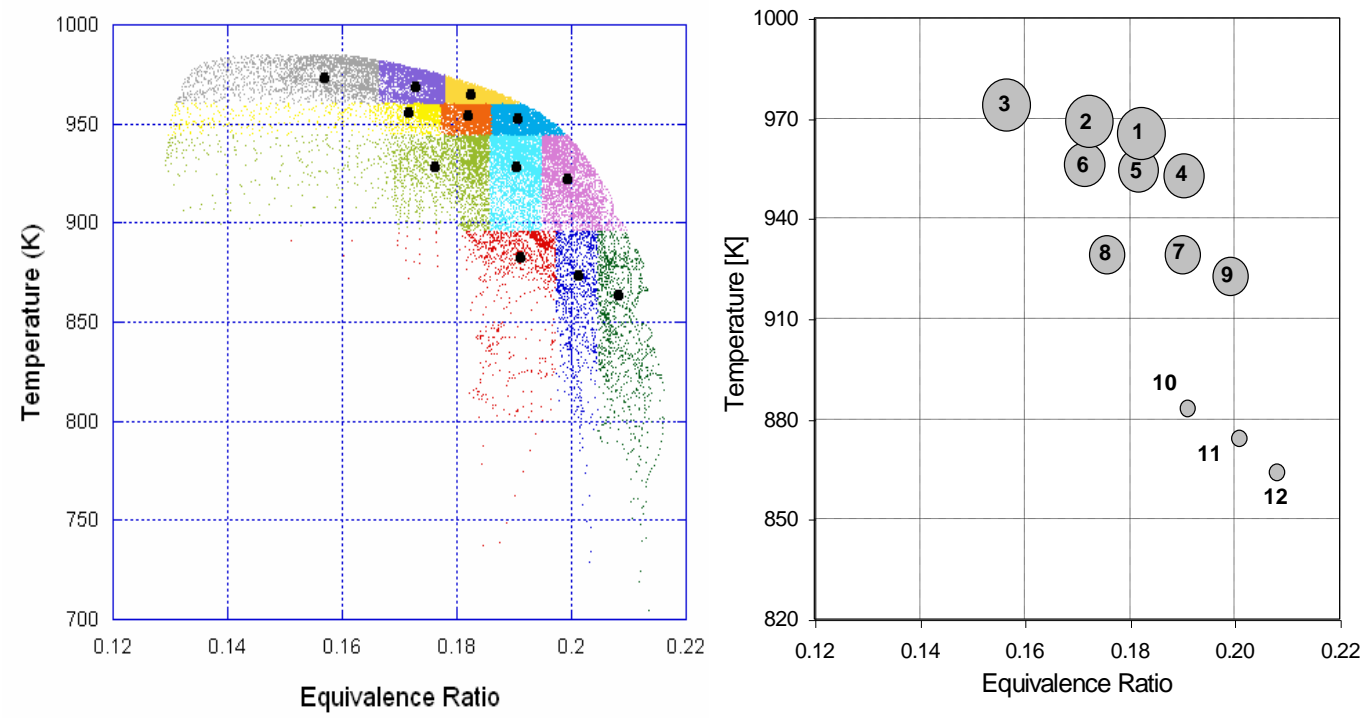

Figure 4.5. At left is shown the temperature-equivalence ratio distribution at $30^{\circ} \mathrm{BTDC}$ with mass divided into zones with centroids at the points indicated. At right the zones are shown mapped by area according to mass. The numbers refer to the order of ignition in the multizone simulation.

in temperature but also in equivalence ratio, as result of incomplete mixing of residual gases at these high residual fractions.

For input into the multi-zone model, the individual cells in Figure 4.5 were ordered first by temperature with the higher temperatures assigned more mass, and then these masses were further divided equally by equivalence ratio. The resulting 12 zones have centroids at the point indicated. The multi-zone computation revealed that the highest temperature zone did not ignite first; instead, zones with relatively high temperature and higher equivalence ratio tended to ignite first. The right side of Figure 4.5 shows the zones with areas proportional to their masses, and numbered in order of ignition.

We further explored the effect of the temperature-equivalence ratio distribution on the overall pressure. To do this we compared the results of the multi-zone combustion model for 
three cases: 1) with temperature/PHI distribution; 2) with temperature distribution only; 3) with single zone as shown in Figure 4.6. These conveniently can be referred to as "cloud", "line" and "point" representations.

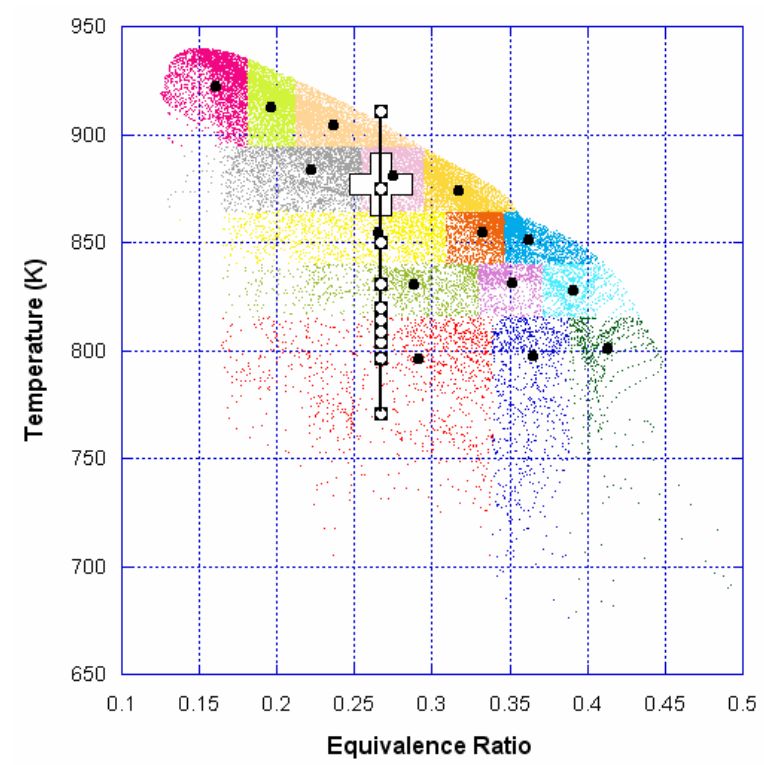

Fig. 4.6. Temperature-Equivalence ratio distributions represented as cloud (colored zones), line (vertical line), and point (cross), for a marginal combustion condition.

The results for a normal combustion event showed little difference between the methods. However, when temperature was scaled down as in Figure 4.6 a marginal combustion condition was encountered where not all the zones ignited. In this case a significant difference was found between representations. Figure 4.7 shows the results for three multi-zone calculations, in which the initial temperature at $30^{\circ}$ BTDC was adjusted upward from $867 \mathrm{~K}$ to $887 \mathrm{~K}$ in $10^{\circ}$ increments. In the three cases shown the line representation always gives an earlier ignition than either the cloud or the point. Referring to Figure 4.6, the line can be seen to extend upwards beyond the actual cloud. As a result, the hottest of the zones has an equivalence ratio equal to the average equivalence ratio of all the cells, which is higher than the actual one for this particular zone. Because of the effect of higher equivalence ratio in facilitating ignition, this point ignites earlier than any point in the cloud. 

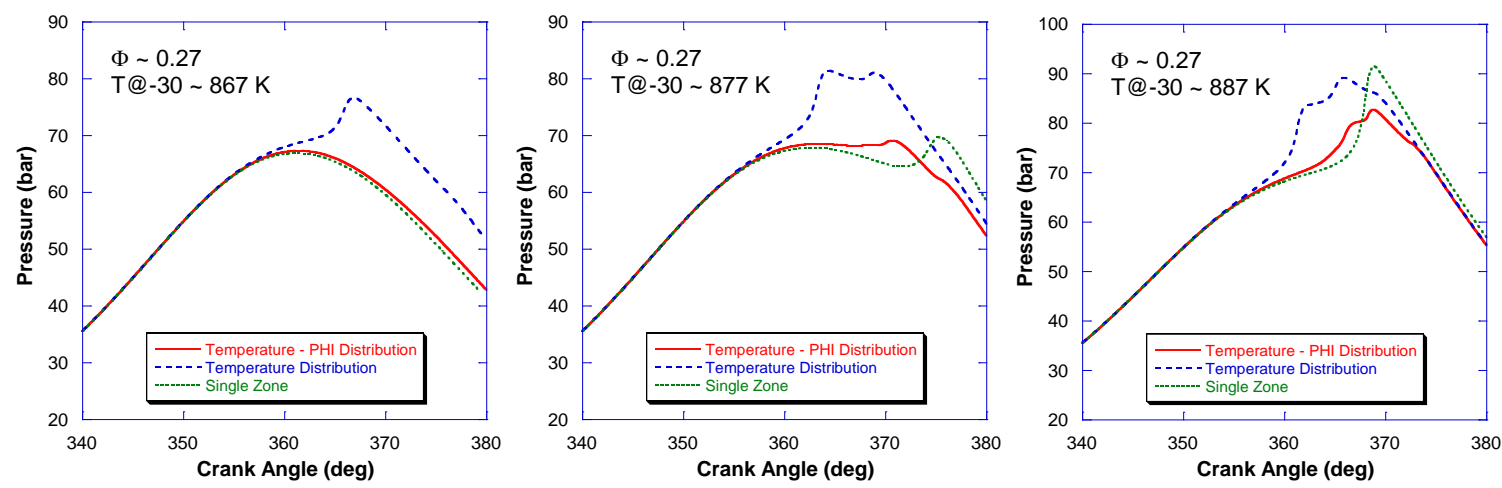

Figure 4.7 Pressure curves calculated with the multizone code under marginal combustion conditions for three different temperatures at $30^{\circ}$ BTDC $(867 \mathrm{~K}, 877 \mathrm{~K}$, and $887 \mathrm{~K})$. Curves comparing "cloud", "line", and "point" representations.

\section{Effect of Valve Strategy on Mixing at High EGR Levels}

In order to isolate the mixing effect on the final in-cylinder temperature / equivalence ratio distribution, a number of negative overlap cases were computed with KIVA-3V with the heat transfer option turned off (adiabatic). Figure 4.8 shows a comparison of the temperature / equivalence ratio distribution for a case with a residual gas fraction of $16 \%$ (negative overlap of $60^{\circ}$ ), obtained with the two different heat transfer treatments.

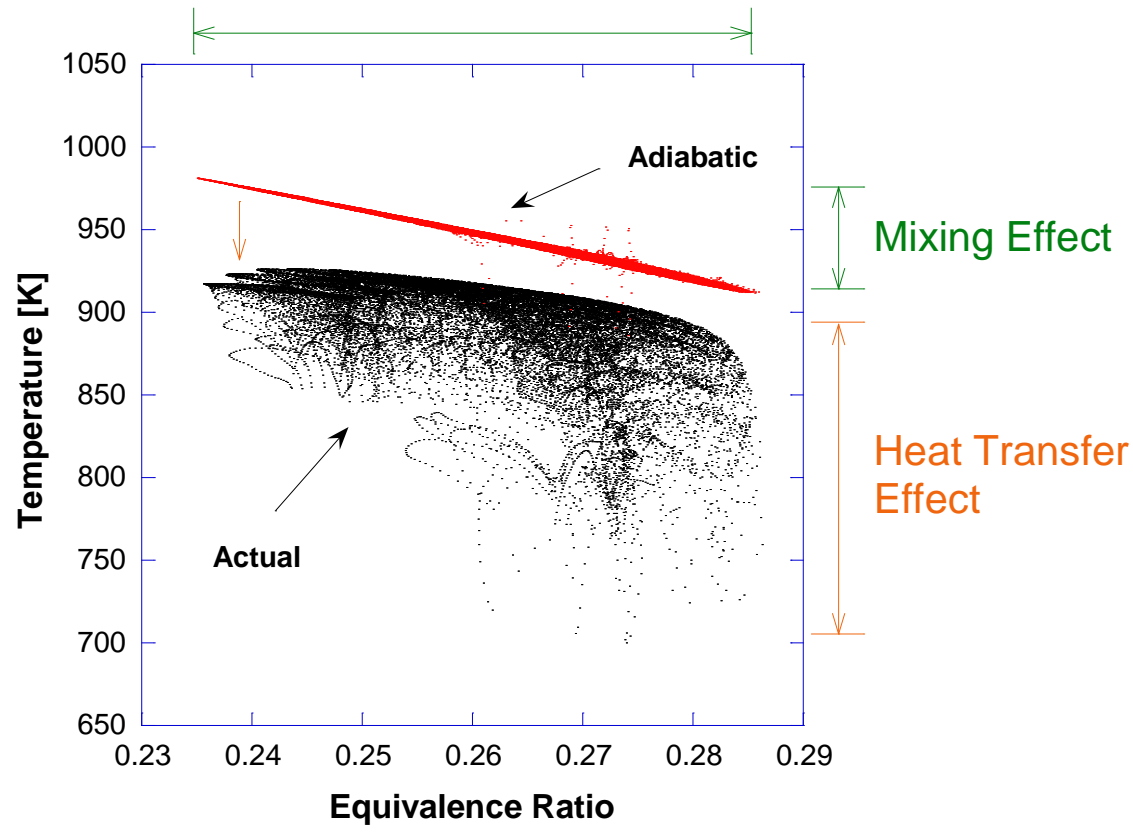

Fig. 4.8 Comparison of Temperature - Equivalence Ratio distributions at $30^{\circ}$ BTDC obtained with normal heat transfer and adiabatic KIVA-3V calculations for negative overlap of $-60^{\circ}$.

The results showed that when there are no heat losses to the cylinder walls, the "cloud" collapses to a single line and there is a linear correlation between the equivalence ratio and the 
temperature. The spread of the equivalence ratio is almost the same for both adiabatic and normal heat transfer cases and is a consequence of the insufficient mixing of the residual gas with the fresh charge. In addition it was found that insufficient mixing can be responsible for temperature differences in the cylinder of 50-150 K, depending on the residual gas fraction. Heat transfer has a dual effect: it lowers the mean (bulk) temperature and causes the deviations from the adiabatic mixing line and the spread of the cloud in the temperature direction.

In light of these findings, we compared two different VVA strategies in terms of mixing of the fresh charge with the residuals. The two strategies were negative valve overlap and rebreathing (secondary opening of the exhaust valve during the intake stroke). The valve timings for each strategy were adjusted so that the same amount of residual gas is trapped. Figure 4.9 shows the temperature / equivalence ratio distributions for a high level of residual gas fraction ( $61 \%)$ obtained with the two methods. The solid line in the plots is the normalized mass density distribution of the equivalence ratio in the cylinder. The narrower this curve, the better the mixing of the fresh charge with the residual gas. Another metric of the mixedness is the C.O.V. of the equivalence ratio $\left(\Phi_{\text {C.O.V. }}\right)$ in the cylinder, defined as the ratio of the standard deviation over the mean value of the equivalence ratio.
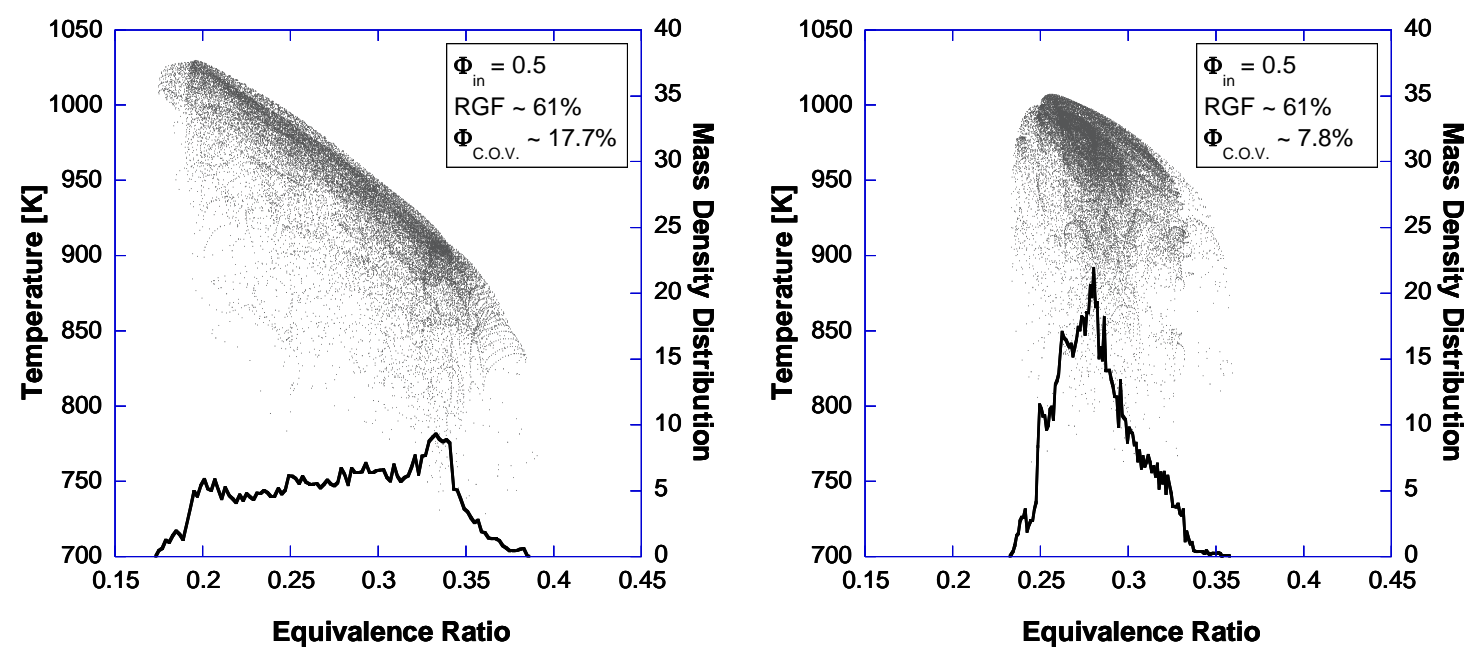

Fig. 4.9. Temperature - Equivalence Ratio distributions at $30^{\circ}$ BTDC for two cases with $61 \%$ residual gas fraction, obtained with negative overlap (left) and rebreathing (right).

It was found that the rebreathing yields more homogeneous mixtures, particularly at high levels of residual gas fraction, as evident by the shape of the clouds and the mass density distributions of the equivalence ratios in Figure 4.9. These differences can be explained by the different way that residual gas is trapped in each case. Negative overlap relies in keeping a large amount of residual gas in the cylinder and when the fresh charge enters the cylinder, it has to mix with the residual in the limited time during intake and compression. In the case of rebreathing, the exhaust gas is pushed out of the cylinder and part of it re-enters during the intake stroke. The two streams, fresh charge and exhaust gas, enter the cylinder simultaneously, having a better chance to mix and produce a more homogeneous mixture. 
The distributions obtained with the two VVA strategies for the different levels of residual gas fraction were used to model the combustion event with the multi-zone code. The zones were initialized using the "cloud" (temperature / equivalence ratio zones), "line" (temperature only zones) and "point" (single-zone) representations, as described in Babajimopoulos et al., (2002). Figure 4.10 shows the results for the two cases presented in Figure 4.9.
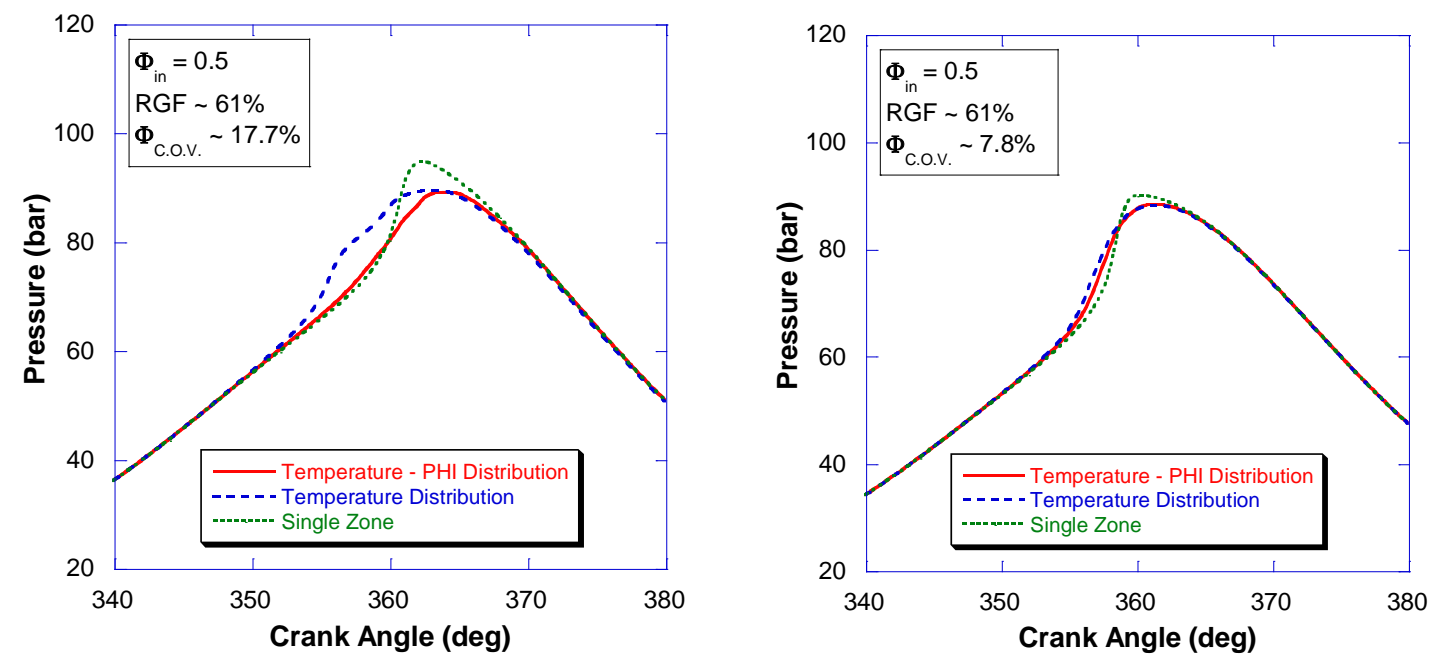

Fig. 4.10. Pressure curves calculate with the multi-zone code for two cases with $61 \%$ residual gas fraction, obtained with negative overlap (left) and rebreathing (right). Curves compare "cloud", "line" and "point" representation.

For the cases with low residual gas fraction, all three representations gave almost identical results. The mixing effect became obvious only at higher levels of residual gas fraction (Figure 4.10), and in particular in the negative overlap case, which had the largest degree of stratification. 


\section{Optical Experiments of Mixing and Reaction(UM)}

Reuss and Sick (2003) have investigated HCCI combustion of n-heptane in an optical engine. Planar laser induced fluorescence images of toluene (an indicator for unburnt fuel zones) and chemiluminescence images of $\mathrm{OH}^{*}$ radicals and emissions in the visible all show a strong dependence of the fuel consumption phasing in time and space on the in-cylinder flow conditions. The image sequences in Figure 4.11 illustrate on an averaged basis (220 consecutive cycles) how the fuel is being consumed. The observed difference in the onset of fuel consumption is reflected in the heat release analysis, shown in Figure 4.12.

\section{Toluene LIF}

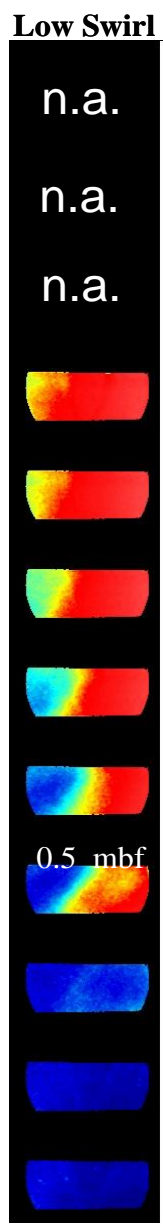

$\underline{\mathbf{a}}$

\section{High Swirl}
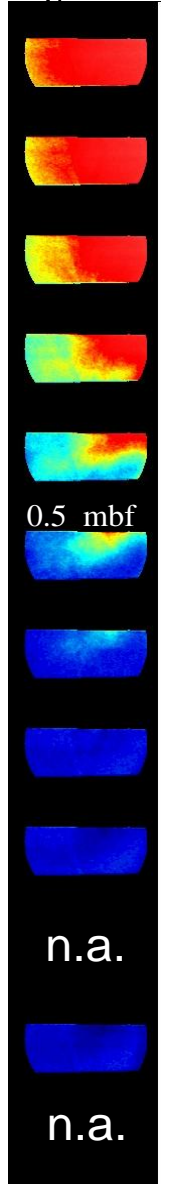

$\underline{\mathbf{b}}$
Chemiluminescence

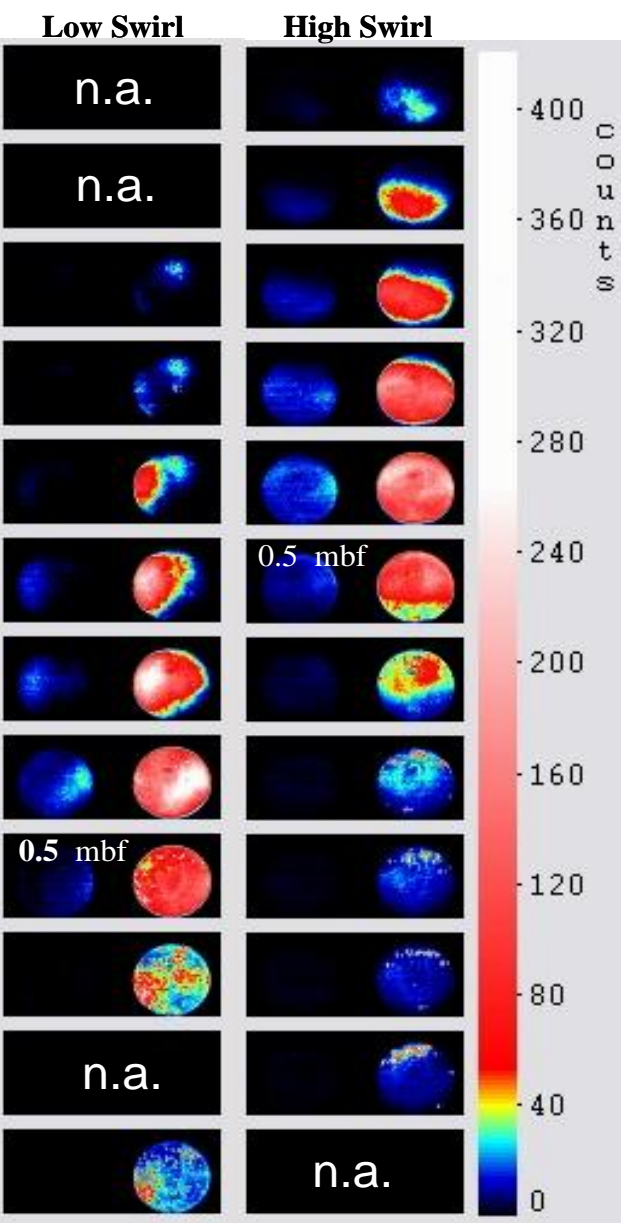

$\underline{\mathbf{c}}$

Figure 4.11: Averaged toluene PLIF (a\&b) and chemiluminescence (c\&d) images. The left of the chemiluminescence image pairs for each swirl setting shows $\mathrm{OH}^{*}$ emissions; the right image shows the emissions in the visible spectral range. (Figure taken from Reuss and sick, 2003) 


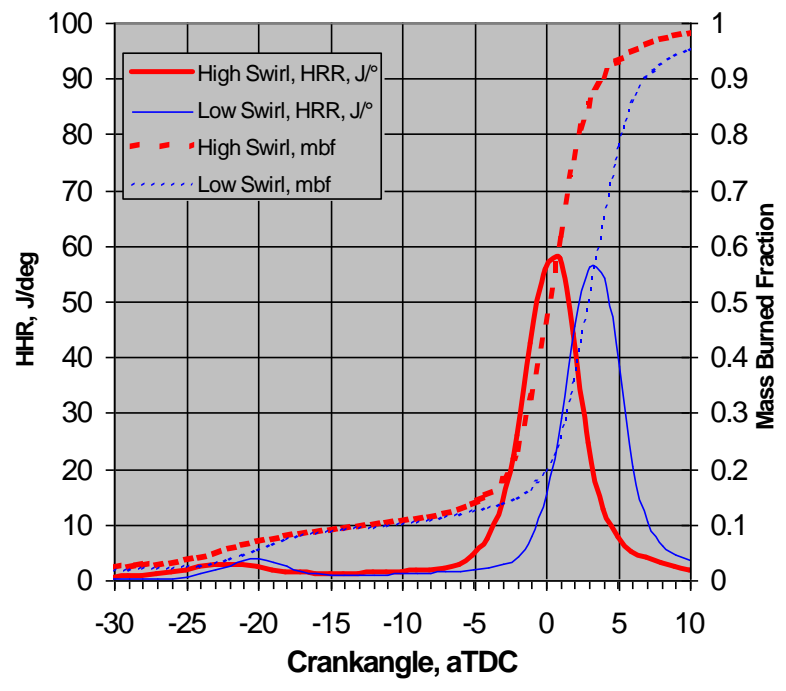

Figure 4.12: Mass burned fraction, mbf and heat release rate, HRR, with low and high swirl. (Figure taken from Reuss and sick, 2003).

Operating conditions are listed in the following.

\section{Engine operating conditions}

- $100 \mathrm{kPa}$ MAP

- Motored at 600 and 2000rpm

- Swirl

- $\operatorname{High}(\mathrm{SR}=5.5)$

- Medium (valve at 36 degrees)

- Low $(\mathrm{SR}=0.8)$

- Measurement plane at $37.2 \mathrm{~mm}$ below firedeck

220 individual vector fields were determined for each operating condition to ensure statistical significance of the results. All measurements were performed with a given setting for the laser pulse separation. While this allows for the most streamlined measurements, it does have an effect on the precision of the results. With decreasing displacement, i. e. velocity, the precision decreases. The impact that this has on derived quantities like kinetic energy and Reynolds stresses is shown in Figure 4.14 .
Information about the flowfield structure and magnitudes as well as turbulence quantities need to be known to investigate the difference in combustion behavior observed for the swirl valve settings as shown in Figures 4.11 and 4.12.

Flow fields and associated turbulence quantities have been measured with digital Particle Image Velocimetry in the optical engine at UM. This engine uses the same cylinderhead as the one reported in [8] to ensure compatibility of the results. The field of view for the images is shown in Figure 4.13.

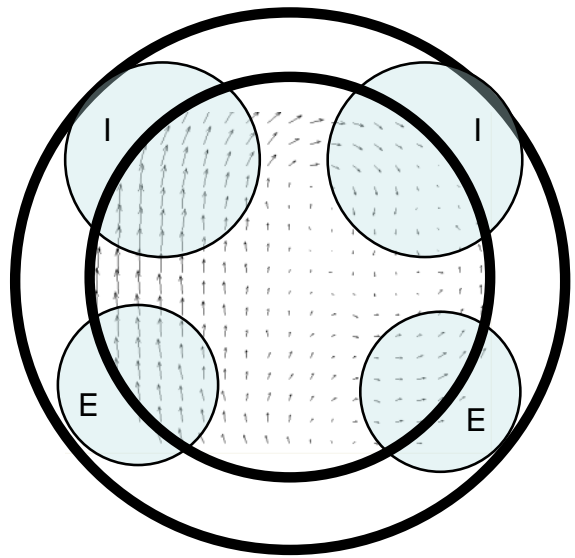

Figure 4.13: Field of view for velocity fields. Valve positions are shown as circles. Left intake can be closed. 


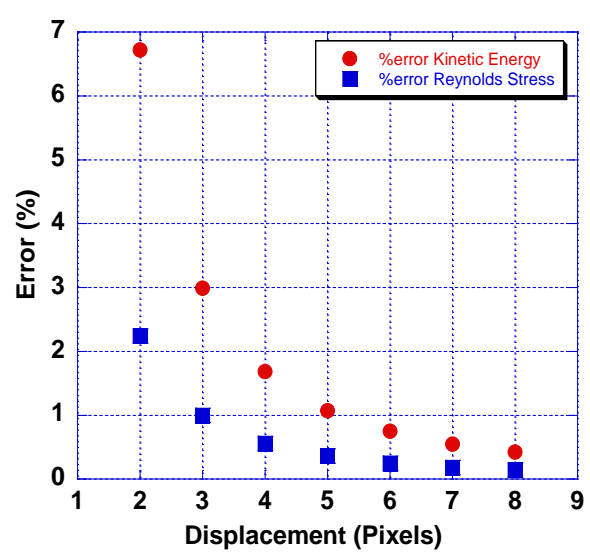

Figure 4.14: Expected changes in precision from PIV derived measurements
Averaged velocity field for engine speeds of 600 and $2000 \mathrm{rpm}$ are shown for all swirl valve settings in Figures 4.15 and 4.16 taken at $98 \mathrm{CAD}$ BTDC. Along with the substantial change in velocity when changing from high to low swirl settings, a pronounced shift of the swirl center is observed initially and at the lowest swirl setting, two swirl centers have developed. The general structure is the same for both engine speeds, however, the position of the swirl centers changes. Paired with the averaged velocity field are the spatial distributions of the mean kinetic energy. Accordingly, there are substantial changes with swirl valve settings. Note the non-linear color coding that is used to illustrate the wide range of energies.
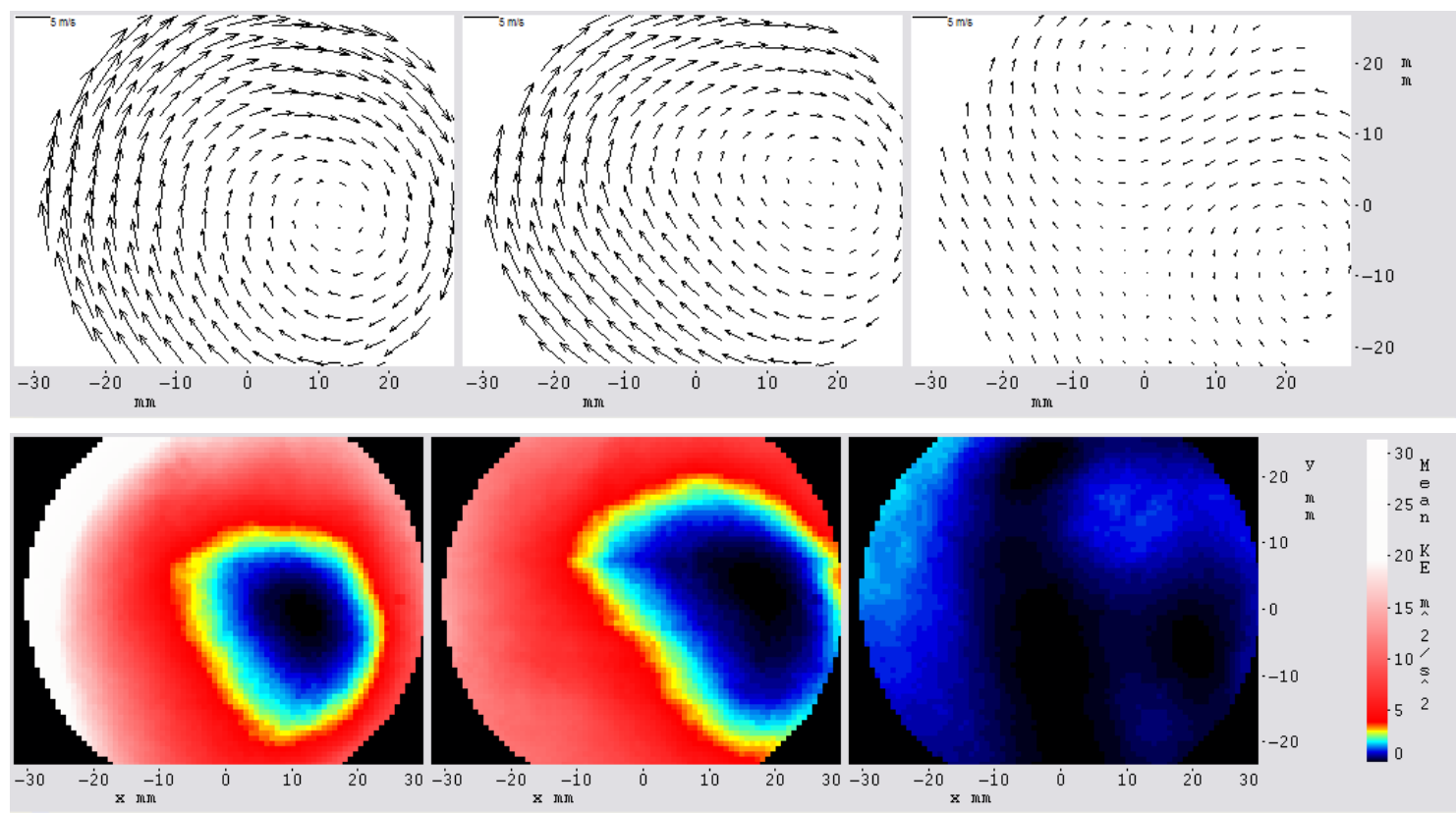

Figure 4.15: Averaged velocity fields (220 cycles) at $600 \mathrm{rpm}$. Swirl valve settings are such that swirl decreases from left to right. Mean kinetic energy distributions are shown in a nonlinear color scale to preserve the same scaling for all images for illustration of the large dynamic range. 


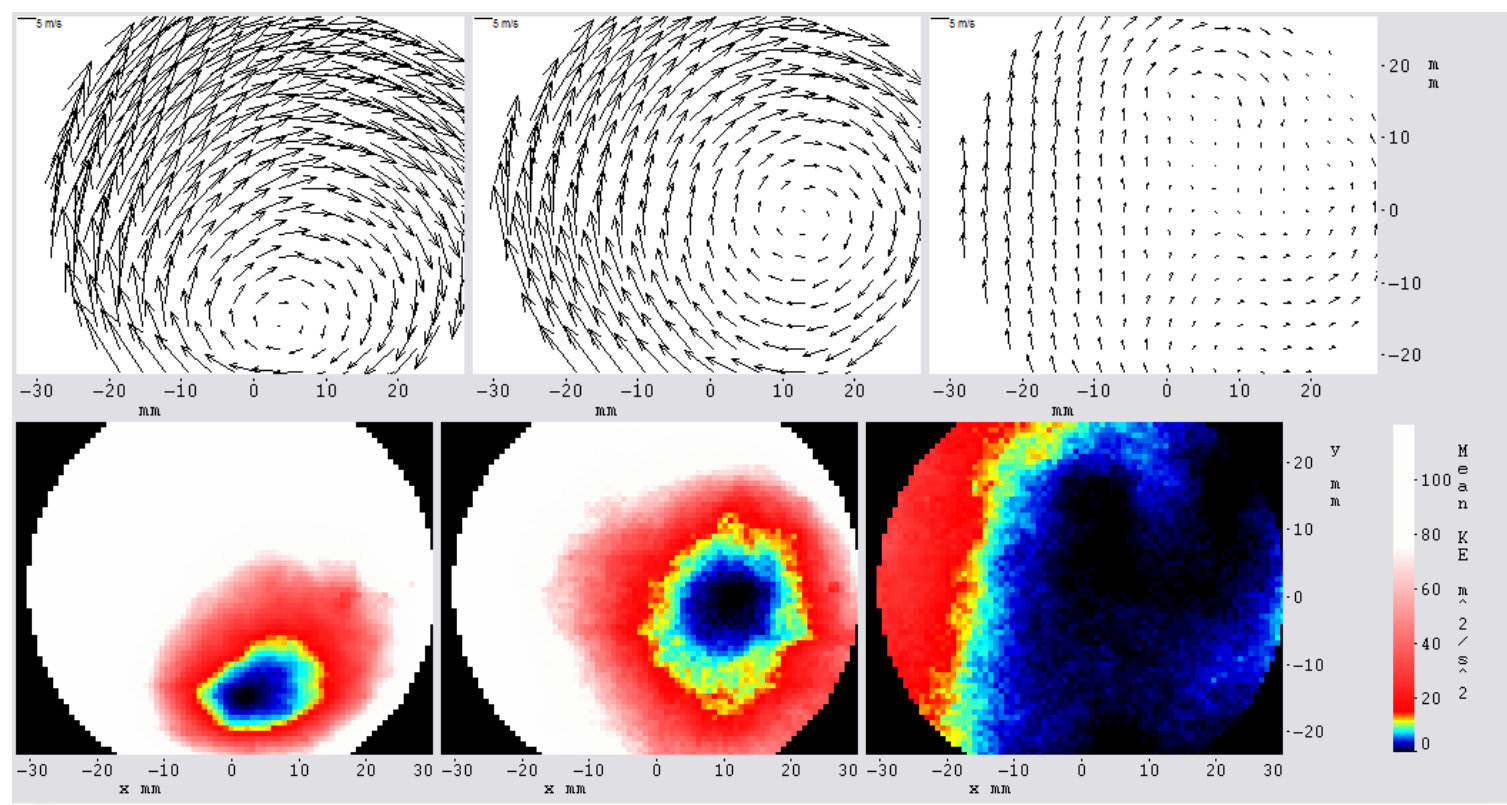

Figure 4.16: Averaged velocity fields ( 220 cycles) at $2000 \mathrm{rpm}$. Swirl valve settings are such that swirl decreases from left to right. Mean kinetic energy distributions are shown in a nonlinear color scale to preserve the same scaling for all images for illustration of the large dynamic range.

Based on heat release analysis we found that in-cylinder swirl affects combustion phasing for low residual gas operation; this is again depicted in Figure 4.17.
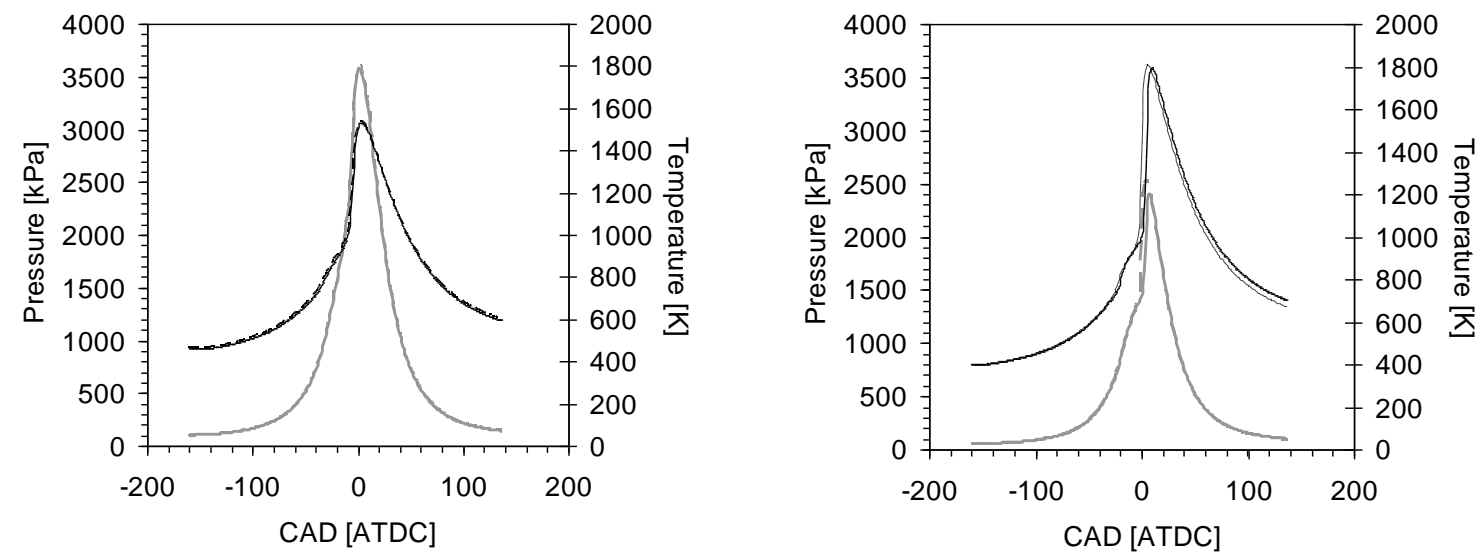

Figure 4.17: Averaged pressure and temperature evolution for high residual gas fraction (LEFT) engine operation is indistinguishable between low and high swirl conditions. For low residual gas fraction (RIGHT) engine operation advanced ignition for high swirl conditions is observed. Pressures are depicted as gray lines, the temperature profiles are shown in black. Solid lines show data for low swirl flow, dashed lines are for data from high swirl flow. 
A more robust image processing strategy was assessed and toluene PLIF images were converted into binarized images that are set to a value of " 1 " where fuel has not been substantially been consumed and " 0 " where high temperature reactions have removed most of the fuel already. This approach eliminates the need for complicated processing of PLIF images while still providing quantitative information for comparison with CFD simulations [12]. The analysis of binarized toluene PLIF images gives a good illustration of how spatial fuel consumption differences and observed combustion performance are related. Figure 4.18 compares averages of 220 binarized phase-locked measurements of toluene LIF images for four operating conditions as denoted in the Figure caption.

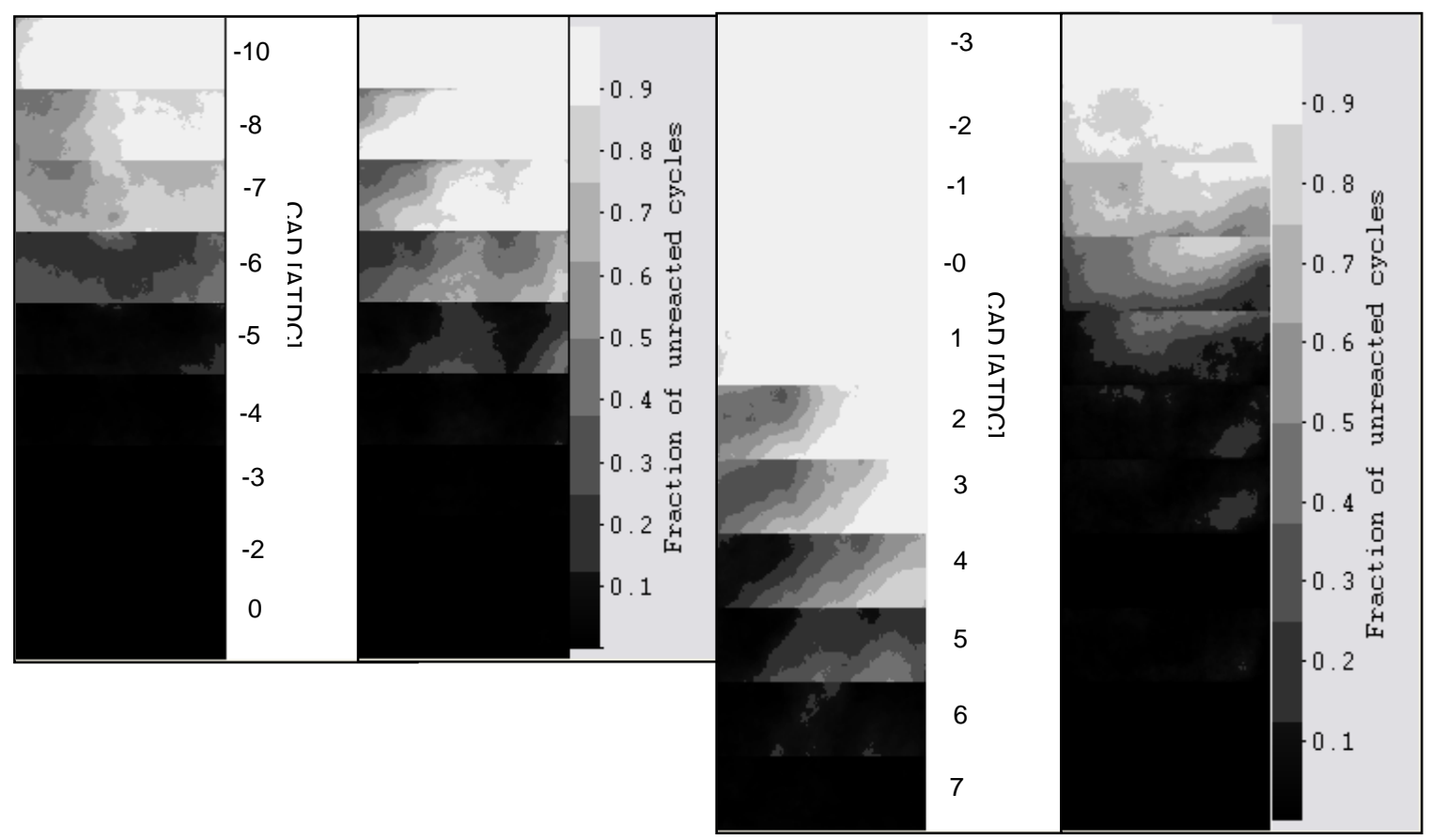

Figure 4.18: Ensemble averages of binarized images from high residual gas fraction engine operation (LEFT block) compared to low residual operation (RIGHT block). The difference between low (left in each block) and high swirl operation is only pronounced for low residual operation, indicating the advanced ignition and combustion for high swirl operation. Also note that the spatial evolution of the fuel consumption changes with swirl.

The phasing differences, as noticed in pressure-related analysis, are obvious from the images in Figure 4.18. Strong spatial preference for fuel consumption evolution is present under all operating conditions.

The observation of single-cycle PLIF images reveals an increase in spatial inhomogeneity towards TDC. In the following, a quantitative assessment of this observation is described. Figure 4.19 shows PLIF images for two operating conditions to illustrate qualitatively how the appearance of the PLIF images changes as the low temperature reaction phase is approached. The "spottiness" of the images can be related to either local fluctuation in temperature and/or fuel concentration. 
A measure of the deviation from perfectly homogeneous conditions is the spatial standard deviation (rms) across an image. Such analysis performed for the LIF images of this study would result in an artificially large standard deviation because of the systematic intensity drop from right to left, due to absorption. However, since arguably the spatial signal intensity modulation before the onset of high-temperature chemistry is small, it was determined that spatial statistical analysis in vertical slices of a few pixels width is not suffering from this shortcoming. These slices are normal to the laser beam direction. Figure 4.20 illustrates the selection of regions-ofinterest for which the spatial average and the spatial standard deviation of the LIF signal intensity were determined. The discussion of the spatial rms values below will be based on the rms normalized by the spatial average that was determined for the same region. This effectively corrected for the effects of laser beam attenuation (note, all based on the argument of small scales) and the normalized rms values become a quantitative measure that can be compared across different engine operating conditions for any crank angle. Note that at later crank angles during the main heat release phase, normalized rms values can exceed 1 manifold because the fluctuations are so large compared to the average value.

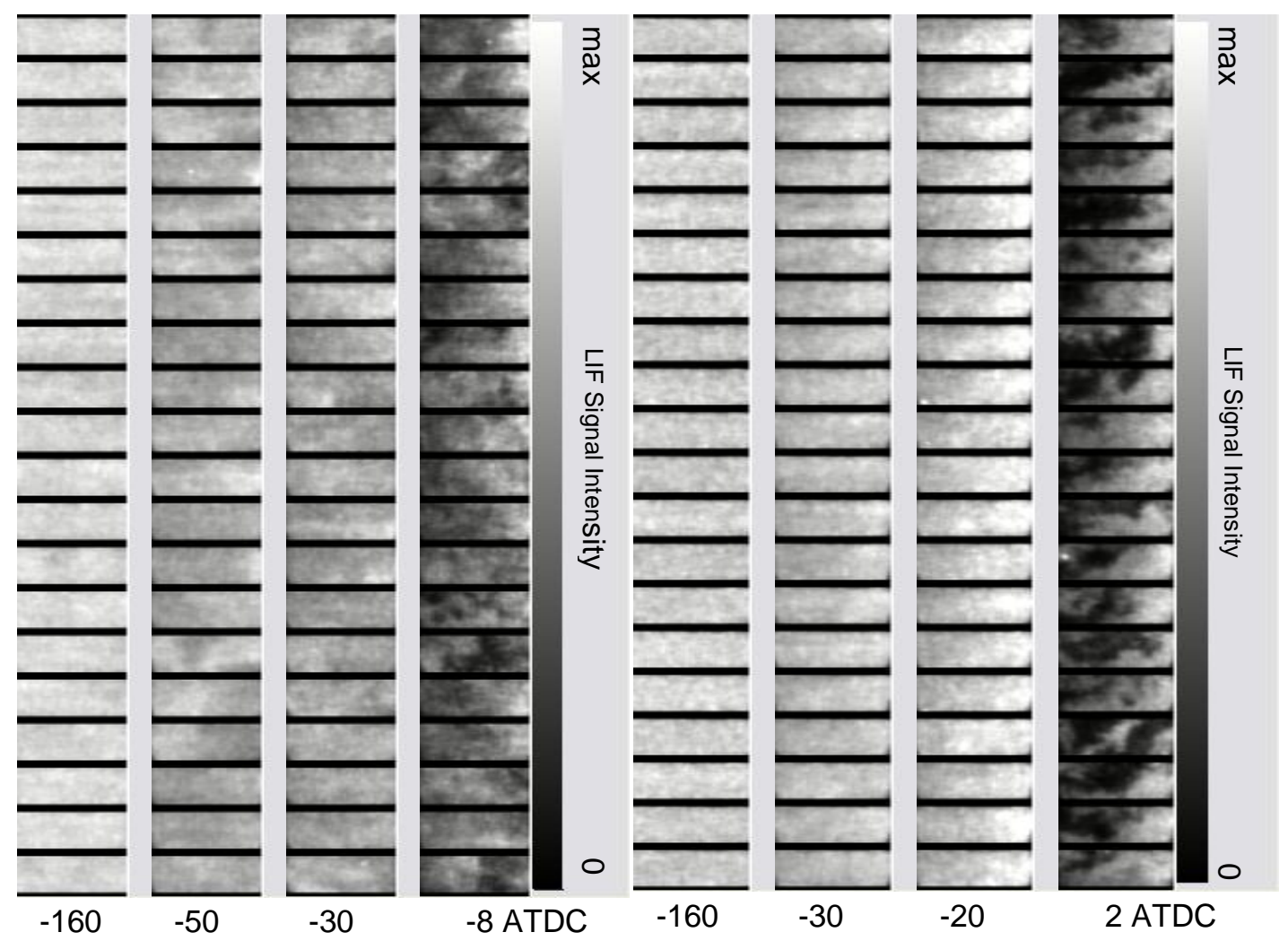

Figure 4.19: Engine operation at high residual, low swirl (LEFT block) and low residual, low swirl (RIGHT block). Toluene PLIF images from 20 consecutive cycles in each column are shown. Note that the relative signal scaling is selected to apply the grayscale from " 0 " to "maximum" separately for each crank angle position. Towards TDC the inhomogeneity increases. The right-most column of each block shows the fuel distribution at $25 \%$ mass fraction burnt. 


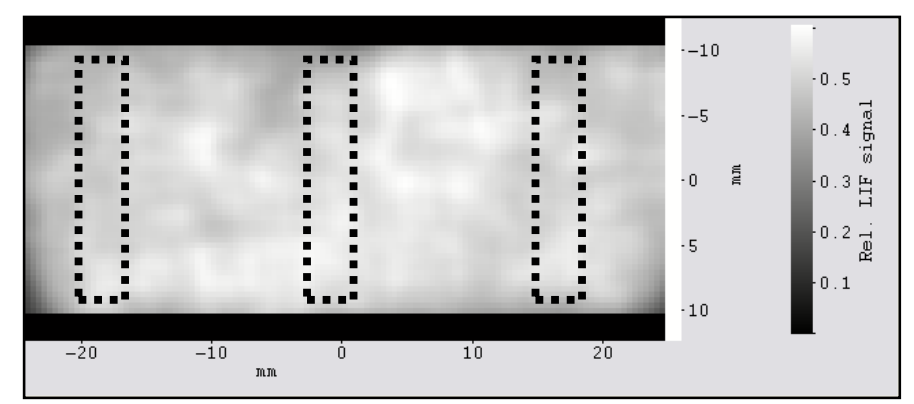

Figure 4.20: Location of the regions-of-interest for the statistical analysis of the LIF images.

A perfectly homogeneous image would have a spatial rms deviation of zero. Signal-to-noise limitations with a LIF detection system and random noise sources will produce a higher rms deviation. The baseline case for the present study is given by motored engine runs without combustion. The results of this analysis are shown in Figure 4.21. Early in the cycle, in one of the rectangles, high rms values are present. At that time and location the intake valve is still open and mixing is not yet complete. At -160 CAD ATDC and beyond the rms across an image for all 220 images that were recorded for each crank angle position is between $2 \%$ and max. $12 \%$.

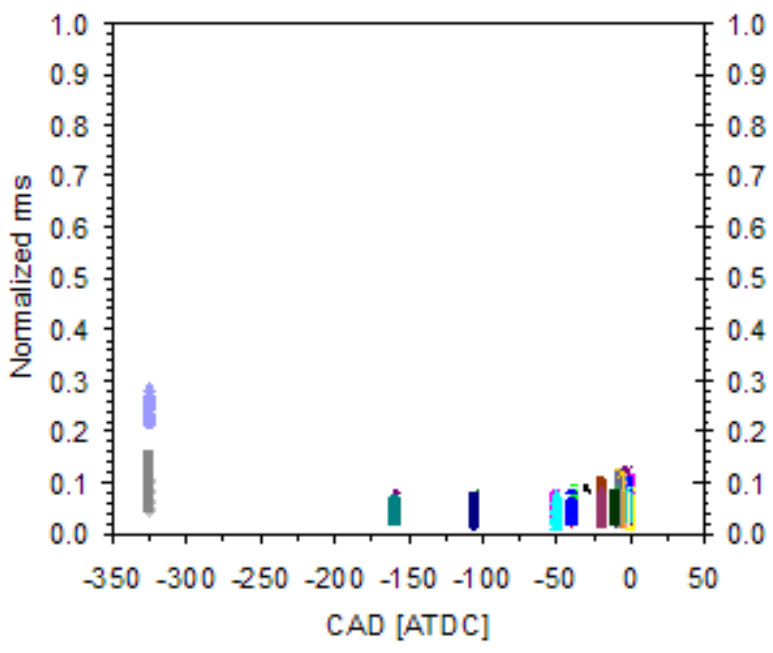

Figure 4.21: Spatial inhomogeneities measured as spatial standard deviation for motored engine operation.
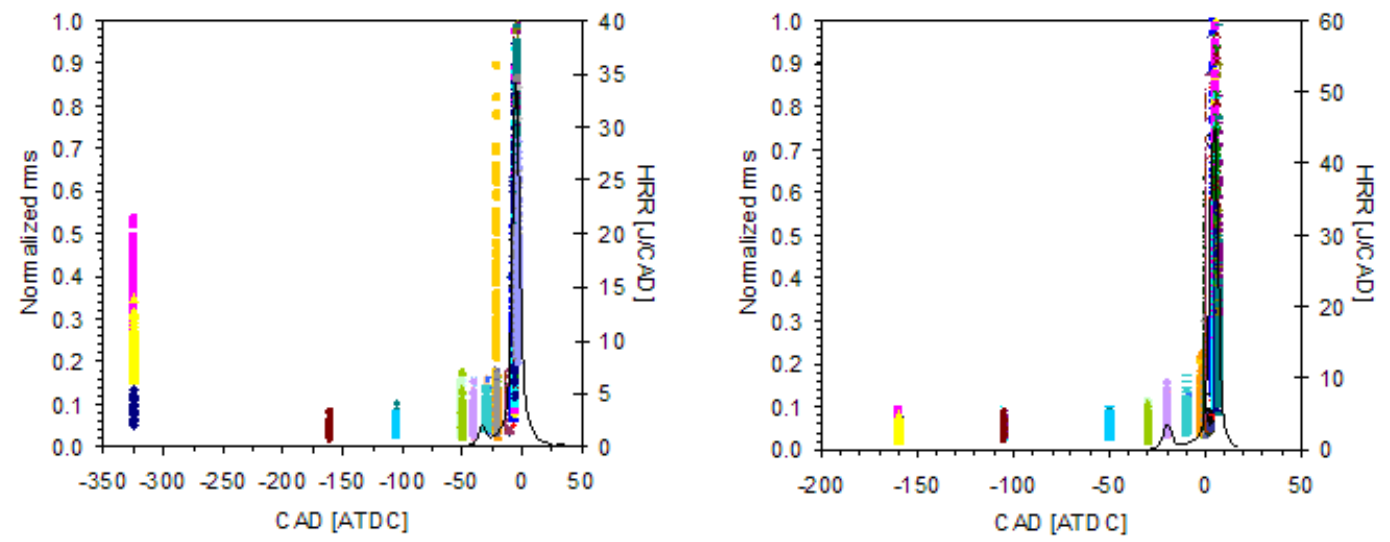

Figure 4.22: Spatial inhomogeneities measured as spatial standard deviation for low swirl, high residual engine operation (LEFT) and for low swirl, low residual engine operation (RIGHT). 
Especially for engine operation with high residual gas fractions, it was observed that the spatial homogeneity decreases beyond what was measured under motored conditions just before LTR phase begins. This is manifested by higher spatial rms values as shown in Figure 4.22. The evaluation of the magnitude of these increased fluctuations in terms of temperature fluctuations is not completed as this stage. Note that the very large fluctuations at -325 CAD ATDC are due to strong mixing of fresh charge with in-cylinder contents while the intake valves are still open.

\section{Developing a TDL Sensor for HCCI Combustion Diagnostics (Stanford)}

The primary goal of this work is to develop a tunable diode laser (TDL) sensor to measure time-resolved $(\mu \mathrm{s})$ temperature and combustion product concentration in an operating internal combustion engine, for the purpose of engine diagnostics and HCCI combustion control. To date, a $1^{\text {st }}$ generation sensor has been developed and applied to the University of Michigan optical engine (developed and utilized by Professor Volker Sick) to measure $\mathrm{T}$ and $\mathrm{X}_{\mathrm{H} 2 \mathrm{O}}$ during motored engine operation and shown in Fig. 4.23.

Modern combustion strategies for internal combustion engines, such as HCCI, rely on accurate knowledge and control of the in-cylinder temperature and gas composition to realize successful operation. Most sensors currently used for IC engine diagnostics either provide an instantaneous 2-dimensional PLIF image of the in-cylinder temperature or fuel concentration, or time-averaged measurements of intake and exhaust gas temperature and composition. Such sensors provide valuable data, but the PLIF measurements are generally limited to one image per cycle, and the intake and exhaust measurements are decoupled from the instantaneous incylinder behavior. Accurate measurements of time-resolved, in-cylinder temperature and gas composition are needed to advance modern IC engine design and development, e.g. through validation of engine simulations, and to aid development of engine control strategies. Diode laser-based absorption diagnostics, based on line-of-sight absorption across the engine cylinder, potentially fulfill these challenging requirements by providing near-real-time measurements of these important quantities.

The results from these first collaborative experiments at UM show promise for accurate measurements of pertinent engine parameters and set the stage for the further development and application of TDL-based sensor to measure additional parameters during fired-engine operation. Currently, a $2^{\text {nd }}$ generation sensor is being developed to improve the accuracy and sensitivity of the measurement in the higher temperature and pressure regimes. 
A schematic of the $1^{\text {st }}$ generation sensor is shown in Fig. 4.24. This multi-wavelength absorption sensor utilizes three distributed feedback (DFB) lasers operating near 1.4 um to probe $\mathrm{H}_{2} \mathrm{O}$ spectroscopic features to determine temperature and $\mathrm{X}_{\mathrm{H} 2 \mathrm{O}}$ with microsecond time response during motored engine operation. A fiber optic guides the multiplexed laser radiation from the

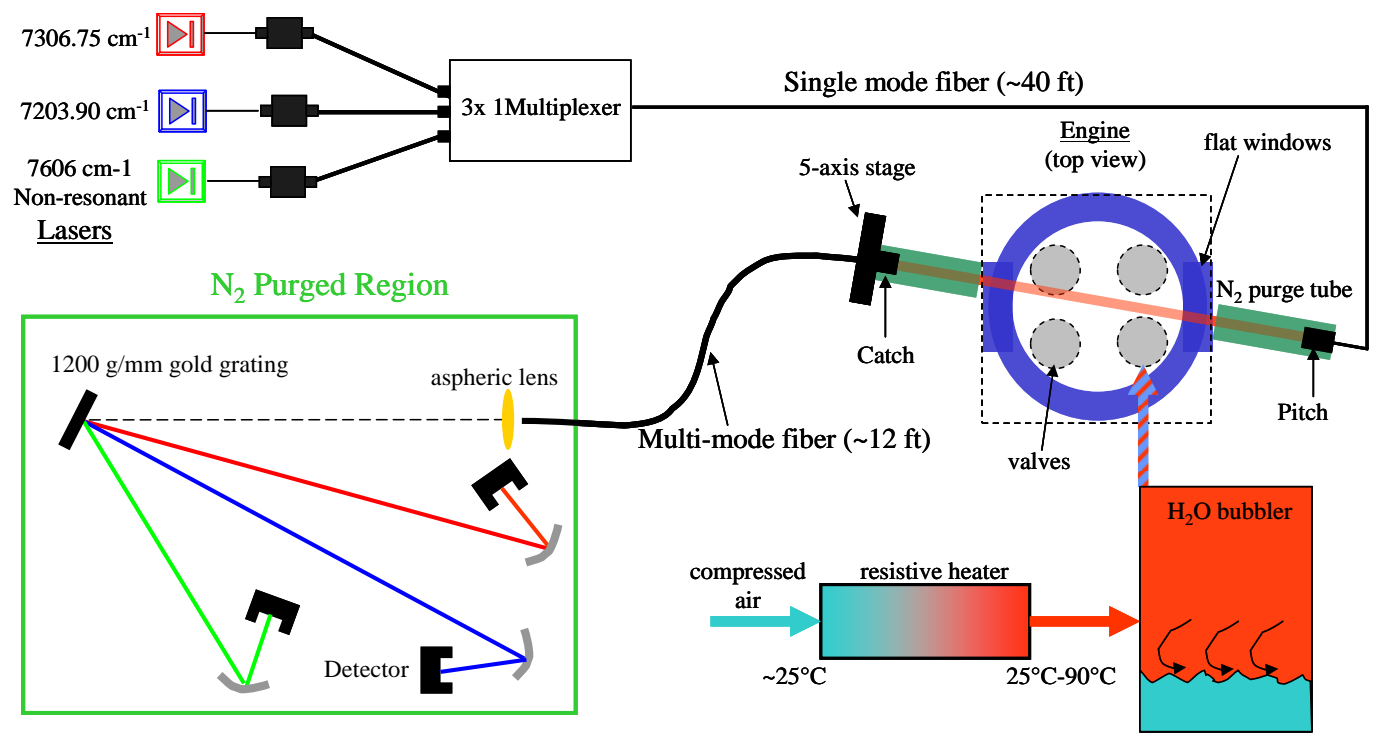

Fig. 4.24 Schematic of Stanford diode-laser based temperature and water concentration sensor applied to the University of Michigan optical engine.

engine control room to the optical engine where it is pitched across the cylinder and collected by another fiber optic. The multiplexed light is then dispersed and the individual intensities are monitored using a PC-based data acquisition system. Water is inducted into the engine cylinder using a gas bubbler system enabling measurements during the compression stroke. Raw data traces of transmitted laser intensity and cylinder pressure are shown in Fig. 4.25. As the gas is compressed and heated, the transmitted intensity at each resonant frequency varies, and through the Beer-Lambert relation temperature and $\mathrm{H}_{2} \mathrm{O}$ concentration are inferred. A sample data trace of temperature during the compression stroke during motored engine operation is shown in Fig. 4.26. The next-generation sensor will utilize additional wavelengths and lower intake water concentrations to reduce the noise appearing near TDC.

The initial results of this collaboration show promise for accurate measurement of critical engine parameters needed for engine diagnostics and combustion control to advance modern IC engine design and development. Future work includes extending the sensor to fired engine operation and to measurements of other species (e.g., fuel, UHCs, $\mathrm{CO}_{2}, \mathrm{CO}$ and $\mathrm{NO}$ ) to investigate pollutant formation and to better quantify and control EGR operation. Other goals include extension of the TDL sensor to quantify non-uniformities (in T and species) across the engine cylinder, and to monitoring of engine parameters at additional locations, such as the intake and exhaust. 

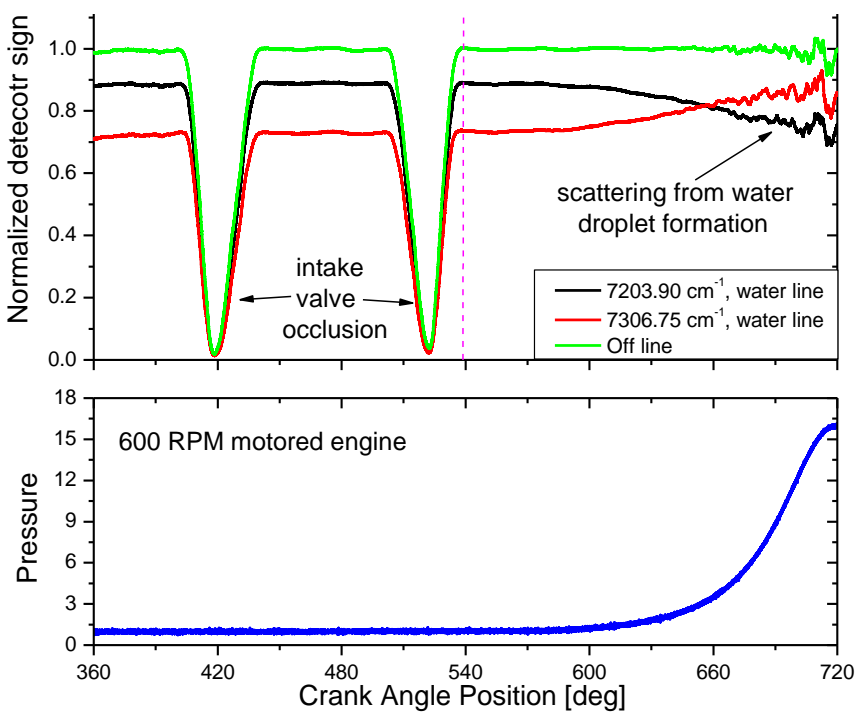

Figure 4.25 Sample raw data trace of laser transmission and pressure during motored engine tests. The attenuation of the transmitted laser power enables time resolved temperature and water concentration measurements throughout the engine cycle.

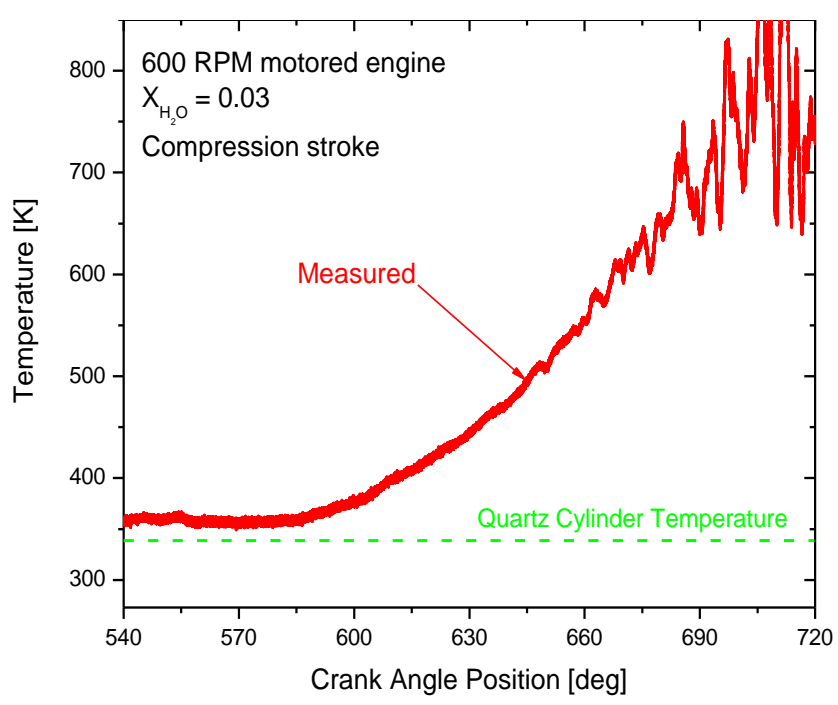

Figure 4.26: Initial results of measured gas temperature during motored engine operation. 


\section{References/Publications}

Aceves, S. M., Flowers, D. L., Chen, J.-Y., Babajimopoulos, A. (2006) Fast Prediction of HCCI Combustion with an Artificial Neural Network Linked to a Fluid Mechanics Code, SAE paper 200601-3298

Aceves, S.M., Flower, D., Martinez-Frias, J., Espinosa-Loza, F., and Dibble, R.W. (2003) Fuel and Additive Characterization for HCCI Combustion. SAE Paper No. 2003-01-1814.

Aceves, S.M., Flowers, D.L., Espinosa-Loza, F., Babajimopoulos, A., Assanis, D.N. (2005) Analysis of premixed charge compression ignition combustion with a sequential fluid mechanics-multizone chemical kinetics model. 2005 SAE Transactions, Journal of Engines (Presented as SAE Paper 200501-0115 at the SAE World Congress, Apr 11-14, 2005, Detroit, MI)

Aceves, S.M., Flowers, D.L., Espinosa-Loza, F., Martinez-Frias, J., Dibble, R.W., Christensen, M., Johansson, B., and Hessel, R.P., (2002) Cylinder Geometry Effect on HCCI Combustion by MultiZone Analysis, SAE Paper No. 2002-01-2869.

Aceves, S.M., Martinez-Frias, J., Flowers, D., Smith, J.R., Dibble, R.W., and Chen, J.-Y., (2002) A Computer Generated Reduced Iso-Octane Chemical Kinetic Mechanism Applied to Simulation of HCCI Combustion, SAE Paper No. 2002-01-2870.

Angelos, J. P., Andreae, M. M. Green, W. H., Cheng, W. K., Kenney, T. and Xu, Y. (2007) Effects of Variation in Market Gasoline Properties on HCCI Load Limits JSAE 20077050, SAE 2007-01-1859 .

Angelos, J. P., Puignou, M., Andreae, M. M., Cheng, W. K., Green, W. H. and Singer, M. A. (submitted) Detailed Chemical Kinetic Simulations of HCCI Engine Transients, International Journal of Engine Research .

Babajimopoulos, A., Assanis, D., and Fiveland, S. B. (2002) Modeling the Effects of Gas Exchange Processes on HCCI Combustion and an Evaluation of Potential Control through Variable Valve Actuation, SAE Paper No. 2002-01-2829.

Babajimopoulos, A., Assanis, D., and Fiveland, S. B. (2002) Modeling the Effects of Gas Exchange Processes on HCCI Combustion and an Evaluation of Potential Control through Variable Valve Actuation, SAE Paper No. 2002-01-2829

Babajimopoulos, A., Assanis, D.N., Flowers, D.L., Aceves, S.M., Hessel, R.P. (2005) A fully coupled computational fluid dynamics and multi-zone model with detailed chemical kinetics for the simulation of premixed charge compression ignition engines. International Journal of Engine Research, vol. 6, no. 5, pp. 497-512

Babajimopoulos, A., Lavoie, G. A., and Assanis, D. N., (2003) Modeling HCCI Combustion with High Levels of Residual Gas Fraction - A Comparison of Two VVA Strategies, SAE Paper No. 2003-013220

Babajimopoulos, A., Lavoie, G.A. and Assanis, D.N. (2007) On the role of top dead center conditions in the combustion phasing of homogeneous charge compression ignition engines. Combustion Science and Technology (in press)

Babajimopoulos, A., Lavoie, George A., and Assanis, Dennis A., (2003) Modeling HCCI Combustion with High Levels of Residual Gas Fraction - A Comparison of Two VVA Strategies, SAE Paper 2003-013220

Bisetti, F., Tham, Y.F., Chen, J.-Y. (2007) Development and assessment of a highly reduced mechanism for isooctane HCCI combustion, paper \#C18, presented at 5th US Combustion Meeting, March 25-28, 2007.

Caton, P. A. et al. (2003) Residual-Effected Homogeneous Charge Compression Ignition at a Low Compression Ratio Using Exhaust Reinduction, Int. J. Engine Res., Vol. 4, No. 3, 2003

Caton, P. A., Song, H. H., Kaahaaina, N. B., and Edwards, C. F. (2005) Residual-Effected Homogeneous 
Charge Compression Ignition with Asymmetric Compression-Expansion Ratios and Elevated Compression Ratio, International Journal of Engine Research, Vol. 6.

Caton, P. A., Song, H. H., Kaahaaina, N. B., and Edwards, C. F. (2005) Strategies For Achieving ResidualEffected Homogeneous Charge Compression Ignition Using Variable Valve Actuation, SAE paper 2005-01-0165, accepted for publication in the SAE Transactions, Journal of Engines.

Chang, J., et al., (2004) New Heat Transfer Correlation for an HCCI Engine Derived From Measurements of Instantaneous Surface Heat Flux, SAE Paper No. 2004-01-2996

Chang, J., et al., (2005) Characterizing the Thermal Sensitivity of a Gasoline HCCI Engine with Measurements of Instantaneous Wall Temperature and Heat Flux. International Journal of Engine Research, Vol. 6, No. 4, 289.

Chang, K., Babajimopoulos, A., Lavoie, G. A., Filipi, Z. S., and Assanis, D. N. (2006) Analysis of Load and Speed Transitions in an HCCI Engine Using 1-D Cycle Simulation and Thermal Networks SAE Paper No. 2006-01-1087.

Chen, J. H., Hawkes, E. R., Sankaran, R., Im, H. G., and Mason, S. D. (2005) Ignition Front Propagation in a Constant Volume with Temperature Inhomogeneities. Combustion and Flame, in press.

Chen, J. H., Hawkes, E., Hewson, J. C., Sankaran, R., and Im, H. G. (2003) The Effect of Turbulent Mixing on Compression Ignition of a Lean Hydrogen/Air Premixture, Fall Meeting of the Western States Section, The Combustion Institute, University of California, Los Angeles, CA, October 20-21.

Chen, J.-Y. Chen, Y.-H., Choi, Y. (2006) Development and Validation of Isooctane Skeletal Mechanisms Based on LLNL Detailed Mechanism International Journal of Vehicle Design, (in press).

Chen, J.-Y., Kolbu, J., Homma, R., and Dibble, R.W. (2003) Optimization of Homogeneous Charge Compression Ignition with Genetic Algorithms, Combustion Science and Technology, 175, 373-392, 2003.

Cheng, A.S., Buchholz, B.A., and Dibble, R.W. (2003) Isotropic Tracing of Fuel Carbon in the Emissions of a Compression-Ignition Engine Fueled with Biodiesel Blends, to be presented at the SAE International Future Transportation Technology Conference, Costa Mesa, CA June 23-24, 2003.

Chesa Rocafort, L., Andreae, M., Green, W.H., Cheng, W.K. and Cowart, J.S. (2005) A Modeling Investigation into the Optimal Intake and Exhaust Valve Event Duration and Timing for a Homogenous Charge Compression Ignition Engine, SAE 2005-01-3746.

Choi, Y. and Chen, J.-Y. (2003) Numerical Modeling of Start-of -Combustion (SOC) in HCCI Engine with Artificial Neural Network (ANN) Presented at the 2003 Fall Western States Meeting of Combustion Instituted, WSSCI paper 03F-69.

Choi, Y. and Chen, J.-Y. (2004) Fast Prediction of Start-Of-Combustion in HCCI with Combined Artificial Neural Networks and Ignition Delay Model Proceedings of the Combustion Institute, 30:2711-2718 (2005).

Curran, H. J., Gaffuri, P., Pitz, W. J. and Westbrook, C. K. (2002) A comprehensive Modeling Study of iso-Octane Oxidation, Combustion and Flame, 129:253-280.

Davidson, D. F., Gauthier, B. M., Hanson, R. K. (2004) Shock Tube Ignition Measurements of IsoOctane/Air and Toluene/Air at High Pressures, Proceedings of the Combustion Institute, 30:1175.

Davidson, D.F., Oehlschlaeger, M.A. and Hanson, R.K. Methyl (2005) Concentration Time Histories during iso-Octane and n-Heptane Oxidation and Pyrolysis, Proceedings of the Combustion Institute 31.

Donovan, M. T., He, X., Zigler, B. T., Palmer, T. R., Wooldridge, M. S., and Atreya, A. (2004) Demonstration of a Free-Piston Rapid Compression Facility for the Study of High Temperature Combustion Phenomena Combustion and Flame, 137, pp. 351-365.

Donovan, M. T., Palmer, T., He, X., Wooldridge, M. S., and Atreya, A. (2002) Demonstration of a FreePiston Rapid Compression Facility to Produce Extended Duration High Temperature/Pressure Reaction Environments for Fundamental Combustion Studies, 2002 Spring Technical Meeting of the 
Canadian Section of the Combustion Institute, May 12-15, 2002, Paper \#5, pp. 1-6.

Eichelberg, G., (1939) Some New Investigations on Old Combustion Engine Problems, Engineering

Filipi, Z., (2004) Experimental Insight into Heat Transfer in a Gasoline HCCI Engine, Presented at the SAE Symposium on HCCI, Berkeley, CA, August 10-11, 2004.

Fiveland, S. B., and Assanis, D. N. (2002) Development and Validation of a Quasi-Dimensional Model for HCCI Engine Performance and Emissions Studies under Turbocharged Conditions, SAE Paper No. 2002-01-1757, 2002.

Flowers, D. L, Aceves, S.M., Martinez-Frias, J., and Dibble, R. W. (2002) Prediction of Carbon Monoxide and Hydrocarbon Emissions in Isooctane HCCI Engine Combustion Using Multi-Zone Simulations, Proceedings of the Combustion Institute, Vol. 29, pp. 687-694

Flowers, D. L., Aceves, S. M., and Babajimopoulos, A. (2006) Effect of Charge Non-Uniformity on Heat Release and Emissions in PCCI Engine Combustion. SAE Paper 2006-01-1363.

Flowers, D., Aceves, S., Martinez-Frias, J., Hessel, R., and Dibble, R.W., (2003) Effect of Mixing on Hydrocarbon and Carbon Monoxide Emissions Prediction for isooctane HCCI Engine Combustion Using a Multi-Zone Detailed Kinetics Solver, SAE Paper No. 2003-01-1821.

Flowers, D.L., Aceves, S.M., Martinez-Frias, J., and Dibble, R.W., (2002) Prediction of Carbon Monoxide and Hydrocarbon Emissions in Iso-Octane HCCI Engine Combustion Using Multi-Zone Simulations, Proceedings of the Combustion Institute, 29, pp. 687-694, 2002.

Funk, C. O., Sick, V., Reuss, D. L., Dahm, W. J. A. (2002) Turbulence Properties of high and low swirl incylinder flows, SAE Technical Paper Series, 2002-01-2841

Gauthier, B. M., Davidson, D. F., and Hanson, R. K. (2004) Shock tube determination of ignition delay times in full-blend and surrogate fuel mixtures. Combustion and Flame, Vol. 139, No. 4, 300-311.

Germano, M., Piomelli, U., Moin, P., Cabot, W. H. (1991) A dynamic subgrid-scale eddy viscosity model, Phys. Fluids A 3, 1760.

Green, W. H. (2004) Computer-Aided Construction of Chemical Kinetic Models, $25^{\text {th }}$ Annual US DOE BES Combustion Research Conference, Airlie, VA June 2004.

Green, W. H., Wijaya, C. D., Yelvington, P. E. and Sumathi, R. (2004) Predicting Chemical Kinetics with Computational Chemistry: Is QOOH $=\mathrm{HOQO}$ Important in Fuel Ignition? Molecular Physics 102, 371-380 .

Green, William H., Wijaya, Catherina D., Yelvington, Paul E., and Sumathi, R. (2003) Predicting Chemical Kinetics: The QOOH HOQO pathway in fuel ignition, FOMMS Meeting in Keystone, CO July 6-11, 2003.

Hamosfakidis, V., Im, H. G., and Assanis, D. N. (2005) A Regenerative Multiple Flamelet Model for PPCI Engine Simulations, Technical Meeting of the Eastern States Section, The Combustion Institute, University of Central Florida, Orlando, FL, November 13-15.

He, X., Donovan, M. T., Palmer, T. R., Zigler, B. T., Wooldridge, M. S., and Atreya, A. (2003) An Experimental Investigation of Ignition Delay Times of Isooctane/O2/N2 Mixtures Using a Rapid Compression Facility, Proceedings of the Third Joint Meeting of the U.S. Sections of The Combustion Institute, March 2003, Chicago, IL.

He, X., Donovan, M. T., Zigler, B. T., Palmer, T. R., Walton, S. M., Wooldridge, M. S., and Atreya, A. (2005) An Experimental Study of Isooctane Ignition Delay Times at HCCI Operating Conditions Using a Rapid Compression Facility, Combustion and Flame, 142, 266-275.

He, X., Walton, S. M., Zigler, B. T., Wooldridge, M. S., and Atreya, A. (2005) A Rapid Compression Facility Study of OH Concentrations of Isooctane Ignition, The $4^{\text {th }}$ Joint Meeting of the U.S. Sections of the Combustion Institute, March 20-23, 2005, Paper \#C09, pp. 1-6.

He, X., Walton, S.M., Zigler, B. T., Wooldridge, M. S., and Atreya, A. (2006) An experimental 
investigation of the intermediates of iso-octane during ignition, submitted to the International Journal of Chemical Kinetics, August 2006, in review.

He, X., Zigler, B. T., Walton, S. M., Wooldridge, M. S., and Atreya, A. (2006) A Rapid Compression Facility Study of $\mathrm{OH}$ Time Histories During Iso-octane Ignition, Combustion and Flame, 145, $552-$ 570.

Hohenberg, G. (1979) Advanced Approaches For Heat Transfer Calculations, SAE paper 790825, Warrendale, PA.

Hohenberg, G., and Killmann, I. (1982) Basic Findings Obtained from Measurements of the Combustion Process, XIX FISITA Congress, Melbourne.

Linan, A. and Williams, F. A. (1995) Autoignition of nonuniform mixtures in chambers of variable volume, Comb. Sci. Tech., 105:245-263.

Mason, S. D., Chen, J. H., and Im, H. G. (2003) Effects of Unsteady Scalar Dissipation Rate on Ignition of Nonpremixed Hydrogen/Air Mixtures in Counterflow, Proceedings of the Combustion Institute, v. 29, pp. 1629-1636 (2002).

Mason, S. D., Chen, J. H., Im, H. G. (2002) Effects of Unsteady Scalar Dissipation Rate on Ignition of Non-Premixed Hydrogen/Air Mixtures in Counterflow, Proceedings of the Combustion Institute, v.29, pp. $1629-1636$

Matthews, J., Santoso, H., and Cheng, W. K. (2005) Load Control for an HCCI Engine, SAE Paper 200501-0150.

Mattison, D.W. Jeffries, J.B. and Hanson, R.K. (2005) Tunable Diode Laser Sensors for Harsh Combustion Environments with Applications to Pulse Detonation Engines and Internal Combustion Engines, Paper A41, Joint Meeting of US Sections of Combustion Institute, Philadelphia, PA, March 2005.

Mattison, D.W., Steeper, R.R., De Zilwa, S., Jeffries, J.B. and Hanson, R.K. (2005) In-Cylinder Gas Temperature Measurements in an HCCI Engine Using a Multiplexed-Wavelength TDL System: Sensor Development and Initial Demonstration Proceedings of the Combustion Institute 31.

Mehresh, P., Souder, J. , Flowers, D., Riedel, U., Dibble, R.W. (2004) Combustion timing in HCCI engines determined by ion-sensor: experimental and kinetic modeling, Proceedings of the Combustion Institute, Vol. 30, p 2701.

Montgomery, C. J., Yang, C., Parkinson, A. R., and Chen, J.-Y. (2006) Selecting the optimum quasisteady-state species for reduced chemical kinetic mechanisms using a genetic algorithm Combustion and Flame, Vol. 144, No. 1-2, 37-52.

Oehlschlaeger, M. A., Davidson, D. F., and Hanson, R. K. (2004) High-Temperature Thermal Decomposition and Iso-Butane and n-Butane Behind Shock Waves, Journal of Physical Chemistry A108: 4247-4253 (2004).

Oehlschlaeger, M.A., Davidson, D.F. and Hanson, R.K. (2004) High-Temperature Thermal Decomposition of iso-Butane and n-Butane Behind Shock Waves, The Journal of Physical Chemistry A 108, 42474253 (2004).

Oehlschlaeger, M.A., Davidson, D.F. and Hanson, R.K. (2005) Thermal Decomposition of Toluene: Overall Rate and Branching Ratio, Proceedings of the Combustion Institute 31.

Papageorgakis, G., (1997) Turbulence modeling of gaseous injection and mixing in DI engines. Ph. D. Thesis, University of Michigan.

Pitsch, H., Barths, H., and Peters, N., (1996) Three-dimensional modeling of NOx and soot formation in DI-Diesel engines using detailed chemistry based on the interactive flamelet approach, SAE Paper 962057.

Reuss, D. L. and Sick, V. (2005) Spatial mixing, ignition and combustion inhomogeneities in HCCI combustion: an imaging study, SAE Technical Paper SAE 2005-01-2122

Reuss, D. L., Sick, V. (2003) Investigation of HCCI Combustion with Combined PLIF Imaging and 
Combustion Analysis, $3^{\text {rd }}$ Joint Meeting of the Combustion Institute Sections, March 2003, Chicago, Ill

Sankaran, R. and Im, H. G. (2005), Characteristics of Auto-Ignition in a Stratified Iso-Octane Mixture with Exhaust Gases under HCCI Conditions, Combustion Theory and Modelling, 9, 417-432.

Sankaran, R., and Im, H. G. (2004) Effects of Mixture Inhomogeneity on the Auto-Ignition of Reactants under HCCI Environment, 42nd Aerospace Sciences Meeting \& Exhibit, Paper No. 2004-1328, Reno, NV, January 5-8.

Sankaran, R., and Im, H. G. (2005) A Theoretical Study on the Effects of Thermal Stratification on the Operating Range of HCCI Engines, 4th Joint Meeting of the U.S. Sections of the Combustion Institute, The Combustion Institute, March 20-23, Drexel University, Philadelphia, PA.

Sankaran, R., Im, H. G., and Hewson, J. C. (2005) Analytical Model for Auto-Ignition in a Thermally Stratified HCCI Engines, Combustion Science and Technology, submitted.

Sankaran, R., Im, H. G., Hawkes, E. R., and Chen, J. H. (2004) A Computational Study on the Ignition of a Lean Hydrogen-Air Mixture with Non-Uniform Temperature Distribution, Spring Technical Meeting of the Central States Section, the Combustion Institute, University of Texas at Austin, Austin, Texas, March 21-23.

Sankaran, R., Im, H. G., Hawkes, E. R., and Chen, J. H. (2005) The Effects of Nonuniform Temperature Distribution on the Ignition of a Lean Homogeneous Hydrogen-Air Mixture, Proceedings of the Combustion Institute, 30, 875-882.

Sankaran, R., Oh, T. K., and Im, H. G. (2003) Ignition and Front Propagation in a Stratified Methane-Air Mixture with Exhaust Gases, Fall Technical Meeting of the Eastern States Section of the Combustion Institute, Pennsylvania State University, State College, PA, Oct. 26-29, 2003.

Sankaran, R., Oh, T. K., and Im, H. G. (2004) Effects of Mixture Inhomogeneity on the Auto-Ignition of Reactants under HCCI Environment, 42nd AIAA Aerospace Sciences Meeting and Exhibit, Jan. 5-8, 2004, Reno, NV.

Sankaran, R., Oh, T. K., and Im, H. G. (2003) Ignition and Front Propagation in a Stratified Methane-Air Mixture with Exhaust Gases, Technical Meeting of the Eastern States Section, The Combustion Institute, Pennsylvania State University, University Park, PA, October 27-29.

Santoso, H., Matthews J. and Cheng, W K. (2005) Managing SI/HCCI Dual-Mode Engine Operation, SAE Paper 2005-01-0162, SAE Congress 2005.

Santoso, H., Matthews, J., and Cheng, W. K. (2005) Characteristics of HCCI Engine Operating in the Negative-Valve-Overlap Mode, SAE Paper 2005-01-2133, 2005.

Schuchardt, K., Oluwole, O., Pitz, W., Rahn, L. A., Green, W. H., Leahy, D. Pancerella, C., Sjoberg, M. and Dec, J. (2005) Development of the RIOT Web Service and Information Technologies to Enable Mechanism Reduction for HCCI Simulations Journal of Physics: Conference Series 16, 107-112 .

Shaver, G. M., Roelle, M. J., Caton, P.A., Kaahaaina, N. B., Ravi, N., Hathout, J-P., Ahmed, J., Kojic, A., Park, S., Edwards, C. F., and Gerdes, J. C. (2005a) A Physics Based Approach to the Control of Homogeneous Charge Compression Ignition Engines with Variable Valve Actuation, Int'l Journal of Engine Research, Vol. 6, No. 4, pp. 361-375, 2005.

Shaver, G. M., Roelle, M., Gerdes, J. C., Caton, P. A., and Edwards, C. F. (2005b) Multi-cycle Modeling of HCCI Engines Utilizing Variable Valve Actuation, ASME Journal of Dynamic Systems, Measurement and Control, Vol. 127, p. 374, 2005.

Sjöberg M., Dec, J. E., Babajimopoulos, A., and Assanis, D. (2004) Comparing Enhanced Natural Thermal Stratification against Retarded Combustion Phasing for Smoothing of HCCI Heat-Release Rates, SAE paper 2004-01-2994.

Sjöberg, M., and Dec, J. E., (2003) Combined effects of fuel-type and engine speed on intake temperature requirements and completeness of bulk-gas reactions for HCCI combustion, 
SAE Paper 2003-01-3173.

Song, J.. Sumathi, R., Yu, J., and Green, W. H., Next Generation Model Construction Software, \& New Approaches to Estimating Rates and Thermochemistry for Combustion, American Chemical Society National Meeting (Fuels Division) Philadelphia, Aug. 2004

Souder, Jason S. (2004) Closed-loop control of a multi-cylinder HCCI engine, Ph.D. Thesis, Department of Mechanical Engineering. University of California, Berkeley.

Tham, Y.F. and Chen, J.-Y., (2003) Recent Advancement on Automatic Generation of Simplified Mechanisms, Presented at the 2003 Fall Western States Meeting of Combustion Institute, WSS-CI paper 03F-49.

Vanzieleghem, B. P. Chryssakis, C. A. Grover, R. O. Sick V. and Assanis. D. N. (2004b) Modeling of Gasoline Direct Injection Mixture Formation with KIVA-3V and Validation with Optical Engine Planar Laser Induced Fluorescence Measurements: Development of an Extended Coherent Flamelet Model. in COMODIA. 2004. Yokohama, Japan.

Vanzieleghem, B. P., Chryssakis, C. A., Grover, R. O., Assanis, D. N., Im H. G., and Sick. V. (2004a) Gasoline Direct Injection Modeling and Validation with Engine Planar Laser Induced Fluorescence Experiments. in 14th International Multidimensional Engine Modeling User's Group Meeting. 2004. Detroit, MI.

Vasu, S.S., Davidson, D.F. and Hanson, R.K. (2005) Shock Tube Measurements and Modeling of Ignition Delay Time in Lean Iso-Octane/Air, Paper 1168, International Symposium on Shock Waves 25, Bangalore, India, July 2005.

Walton, S. M., He, X., Zigler, B. T., and Wooldridge, M. S., (2006) An Experimental Investigation of the Ignition Properties of Hydrogen and Carbon Monoxide Mixtures for Syngas Turbine Applications, accepted to the $31^{s t}$ Proceedings of the Combustion Institute, (in press).

Walton, S. M., He, X., Zigler, B. T., Wooldridge, M. S., and Atreya, A. (2005) Demonstration of Distinct Ignition Regimes Using High-Speed Digital Imaging of Iso-octane Mixtures, The $4^{\text {th }}$ Joint Meeting of the U.S. Sections of the Combustion Institute, March 20-23, 2005, Paper \#A29, pp. 1-6.

Walton, S. M., He, X., Zigler, B. T., Wooldridge, M. S., and Atreya, A. (2006) An Experimental Investigation of Iso-octane Ignition Phenomena, accepted to Combustion and Flame, in press.

Woschni, G. (1967) Equation for the Instantaneous Heat Transfer Coefficient in the Internal Combustion Engine, SAE Paper No. 670931

Yelvington, P. E., and Green, W. H. (2003) Prediction of the Knock Limit and Viable Operating Range for a Homogeneous-Charge Compression-Ignition (HCCI) Engine, SAE Paper No. 2003-01-1092.

Yelvington, P. E., Bernat i Rollo, M., Liput, S., Tester, J.W., Green, W. H., and Yang, J. (2004) Predicting Performance Maps for HCCI Engines, Combustion Science \& Technology 176, 1243-1282 (2004).

Zigler, B. T., Walton, S. M., Wiswall, J. T., and Wooldridge, M. S. (2006) Crank-angle resolved imaging of homogeneous charge compression ignition phenomena in single-cylinder research engine, The Central States Meeting of the Combustion Institute, May 21-23, 2006, Paper \#, pp. 1-5. 